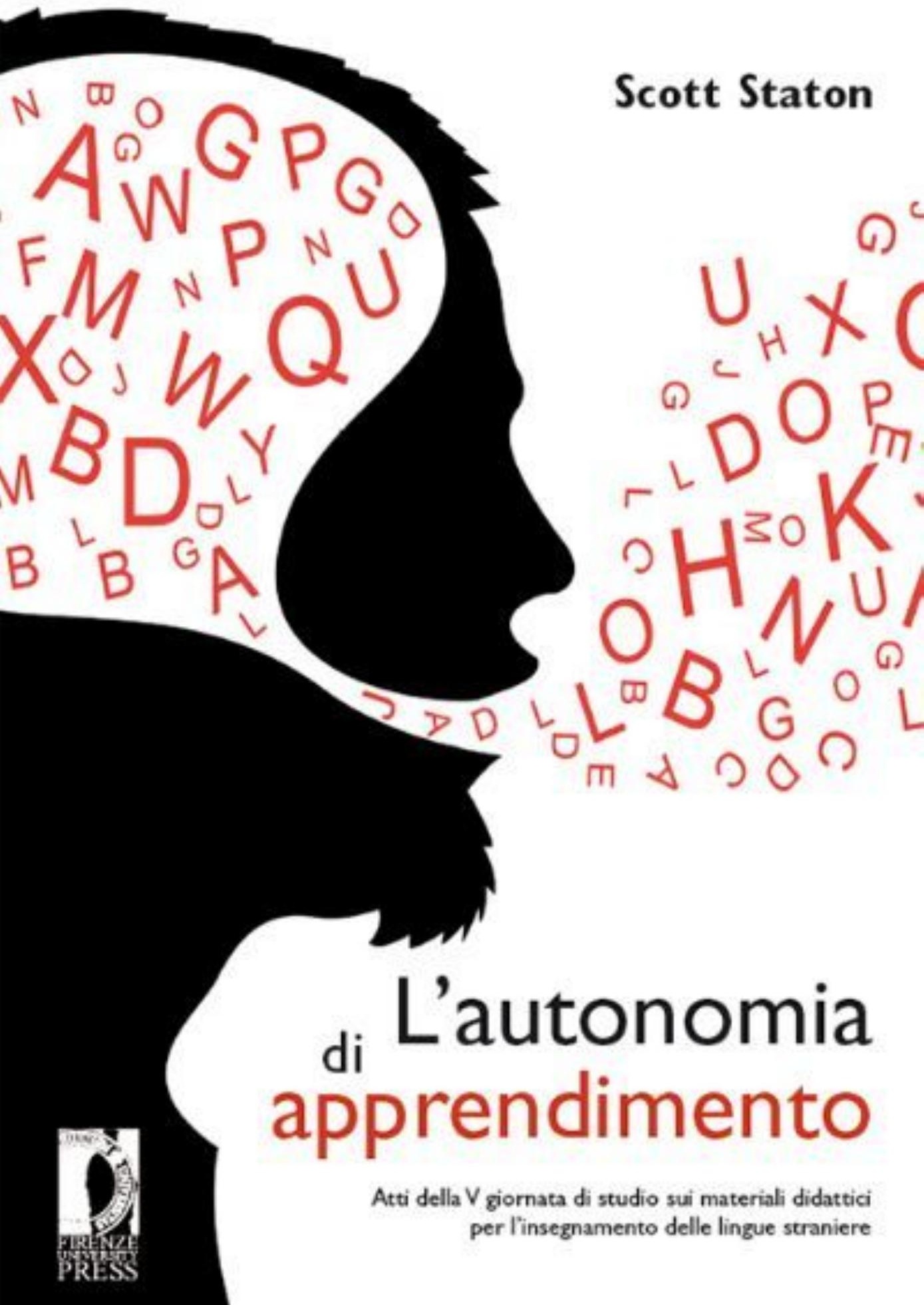


Proceedings e report 



\title{
L'autonomia di apprendimento
}

Atti della $\mathrm{V}$ giornata di studio sui materiali

didattici per l'insegnamento delle lingue straniere

\author{
a cura di \\ Scott Staton
}

FIRENZE UNIVERSITY PRESS

2010 
L'autonomia di apprendimento : atti della V giornata di studio sui materiali didattici per l'insegnamento delle lingue straniere / a cura di Scott Staton. - Firenze : Firenze University Press, 2010.

(Proceeding e report ; 58)

http://digital.casalini.it/9788884537744

ISBN 978-88-8453-774-4 (on line)

Immagine di copertina: (C) Orson | Dreamstime.com

Progetto grafico di Alberto Pizarro Fernández

C) 2010 Firenze University Press

Università degli Studi di Firenze

Firenze University Press

Borgo Albizi, 28, 50122 Firenze, Italy

http://www.fupress.com/

Printed in Italy 
SOMMARIO

INTRODUZIONE

María Carlota Nicolás Martínez

PROJECT WORK TEATRALE

Lucia Alessio

1. Introduzione

2. La lingua del teatro

3. La lingua degli apprendenti

4. La lingua nel laboratorio teatrale 8

5. L'aspetto interculturale $\quad 10$

Appendice 1

Appendice 2 11

Appendice 3 12

$\begin{array}{ll}\text { Bibliografia } & 14\end{array}$

“FIRENZE COM'ERA". PROGETTO PER L'APPRENDIMENTO AUTONOMO DELLA LINGUA ITALIANA

Grazia Giannelli, Caterina Sani

1. Introduzione 17

2. Inquadramento teorico-metodologico 17

3. Il project work 20

4. Conclusioni 28

Bibliografia $\quad 29$

AUTONOMIA DI APPRENDIMENTO LINGUISTICO IN

CONTESTO MIGRATORIO: IL CASO DEI LAVORATORI DI

ORIGINE STRANIERA

Raymond Siebtcheu

1. Introduzione

2. Autonomia di apprendimento e auto-apprendimento 32

3. Il binomio lingua e immigrazione 
4. Il lavoratore di origine straniera 33

5. La questione della lingua 34

6. Il comportamento linguistico del lavoratore di origine straniero $\quad 36$

7. La questione del materiale didattico 39

8. Dall'autonomia di apprendimento all'autonomia linguistica 40

9. Conclusioni 43

Bibliografia 44

L'AUTONOMIA DI APPRENDIMENTO DELLE LINGUE NEL

BIENNIO UNIVERSITARIO: LA DIDATTICA ALL'INSEGNA DEL

COSTRUTTIVISMO CON CLASSI VIRTUALI E RICERCHE ONLINE 49

Christiane Büchel

1. Introduzione

2. I nuovi compiti dell'insegnante: educazione, costruzione, istruzione

3. Educare all'autonomia per l'apprendimento significativo 52

4. La creazione di ambienti/classi/aule virtuali 53

5. Esperienze didattiche dell'insegnamento della lingua tedesca con base informatica

Sito e bibliografia (scelta) 62

MARCOS DE REFERENCIA PARA EL APRENDIZAJE PERSONAL 65

Pedro Pablo Sánchez-Villalón, Manuel Ortega, Asunción Sánchez-Villalón

1. Introducción

2. Marcos de Referencia 66

3. AIOLE y el ePortfolio 70

4. Conclusiones 72

Referencias $\quad 73$

EL AULA VIRTUAL DE ESPAÑOL (AVE): UN ENTORNO PARA EL APRENDIZAJE AUTÓNOMO

María del Pilar Hernández Mercedes

1. Introducción 75

2. ¿Qué es exactamente el ave?

3. Estructura 77

4. El entorno de aprendizaje $\quad 77$

5. Materiales didácticos 79

6. Modalidades de explotación 81

7. Autonomía de aprendizaje y Aprendizaje autónomo 83

8. El AVE y las instituciones 86

9. Conclusiones 87

Bibliografía básica y Webliografía $\quad 88$ 
IL TUTORATO PER L'AUTOAPPRENDIMENTO RISERVATO AI PRINCIPIANTI ASSOLUTI DI LINGUA TEDESCA. VALUTAZIONE DI UN'OFFERTA DIDATTICA AGGIUNTIVA

Gudrun Bukies

1. Introduzione

2. L'insegnamento delle lingue alla Facoltà di Lingue e Letterature Straniere dell'Università di Cagliari

3. Il tutorato per l' autoapprendimento riservato ai principianti assoluti di lingua tedesca

4. Il questionario - La valutazione del tutorato da parte dei frequentanti

5. L'esame di Lingua Tedesca I: alcuni dati della sessione estiva 2007

6. Conclusioni

Riferimenti bibliografici

105

THE ROLE OF LANGUAGE AWARENESS AND LEARNER COUNSELLING IN DEVELOPING LEARNER AUTONOMY: TOWARDS A MORE FLEXIBLE APPROACH TO TEACHING AND LEARNING

Luisa Panichi

1. Introduction

2. Language Awareness and Learner Counselling 108

3. Learner autonomy and authenticity

4. Learner autonomy and agency

5. Examples

6. Conclusions and cautionary remarks

References

ORDINARY LANGUAGE METAPHORS: A PLAN

FOR A CORPUS-BASED RESEARCH PROJECT

Scott Staton

1. Introduction

2. Getting to know the theory

3. Using the corpus

4. Choosing a hypothesis to test

5. Bridging the gap between abstract and concrete

6. The research project

7. Conclusion

Appendix 1

Appendix 2

References 
A NEW CONCEPTUAL METAPHOR: RELATIONSHIPS ARE ARTEFACTS

Manuela Magnoni

1. Introduction

2. Method of analysis

3. The conceptual metaphor RELATIONSHIPS ARE ARTEFACTS

4. Conclusions

Appendix

Works Cited

BREVE STORIA LEGISLATIVA, GIURIDICA E CONTRATTUALE

DEGLI INSEGNANTI UNIVERSITARI DI MADRELINGUA IN ITALIA

John Gilbert

1. Premessa

153

2. La storia

153

3. Conclusioni 


\title{
INTRODUZIONE
}

\author{
María Carlota Nicolás Martínez \\ Università degli Studi di Firenze
}

È sempre un privilegio potere affrontare argomenti che sono in continua evoluzione, caratterizzati da sfaccettature e da sottili sfumature, e con ciò mi riferisco all'argomento dell'apprendimento autonomo o dell'autonomia di apprendimento oggetto di questo libro.

Si sa che l'argomento è aperto, dal momento che sono ancora oggetto di chiarimenti i termini e l'uso di prestiti stranieri con i quali si denomina questo ambito di studio all'interno della didattica delle lingue; a tal proposito in questo libro si offrono definizioni e delimitazioni tra le diverse denominazioni in modo da chiarire la posizione di ogni autore nel modo di intendere l'argomento, ma si ripercorrono anche i cambiamenti avvenuti nelle tradizioni di questi studi, citando sempre le fonti per chiarire le delimitazioni concettuali dei termini.

Oltre alla relazione fra i concetti di apprendimento e di autonomia, in questa pubblicazione si mostrano le applicazioni di questo modo di insegnare/apprendere, si propongono modelli o percorsi di lavoro concreto per chi volesse mettere in pratica un insegnamento/apprendimento non centrato sulla figura del docente, ma gestito in gran parte dall'apprendente.

Ma il valore di questo libro, non inteso come valore concreto di ogni articolo ma come pubblicazione che si considera di interesse, sta nel fatto che mostra una soglia ormai superata nella didattica delle lingue, e cioè che in nessun momento nessun autore mette ormai più in discussione l'uso della tecnologia o del materiale informatico per raggiungere il suo progetto di lavoro. Pertanto i materiali informatici o in formato informatico, per il fatto che sono materiali condivisibili, da professore a allievo ma spesso anche direttamente fra gli allievi, sembrano ormai essere presupposti imprescindibili nella metodologia di apprendimento legata all'autonomia. Questo mi sembra il punto di forte interesse che emerge dall'opera: un insegnamento legato alle nuove tecnologie si dà per scontato e sono stati necessari tanti anni per arrivare a questo presupposto.

Un altro nuovo presupposto che prende forma in diversi di questi lavori è che la figura dell'insegnante è ormai molto diversa dalla figu- 
ra tradizionale, i contenuti non vengono da lui ma sono informazioni raccolte generalmente dagli studenti in rete o anche sul campo nella vita quotidiana. Le ragioni sembrano emergere anche dai cambiamenti di costume: oggi la generazione degli adulti cresciuti senza computer vede con sorpresa diminuire la comunicazione diretta nei modi di socializzare dei giovani, e le modalità dell'insegnamento si devono adattare ai tempi. L'uso dei blog, delle chat, di Facebook o di wikipedia sono da valorizzare in funzione delle esigenze di formazione, e il modo (all'apparenza) frammentario di informarsi in rete può essere valorizzato ai fini dell'apprendimento se, e solo se, il professore approfitta di questo tipo di abitudine alla ricerca e crea un progetto stimolante in modo di fare svolgere un lavoro continuo ed esauriente all'apprendente. L'abitudine di passare da una pagina web ad un'altra può essere considerato un modo attuale di acquistare conoscenza e si può sfruttare per introdurre - come si vede nei progetti di apprendimento che si propongono in questo libro - la possibilità di passare da un argomento all'altro, purché interconnessi, perché il rischio di fare ricerche che rimangono tasselli isolati oltre a non offrire in generale una solida acquisizione di conoscenza non offre neanche un modo di continuità, e di invito alla continuità, che è uno dei principi più importanti per arrivare a rendere efficace l'autonomia nell'apprendimento. È pertanto necessario assicurare che si otterranno risultati anche attraverso la messa in relazione delle ricerche fatte, anche come mezzo per favorire la continuità del lavoro dell'apprendere. Inoltre, il mantenere un filo di contatto con altri apprendenti o con il professore o il tutor è molto utile per la necessaria continuità del lavoro dell'apprendente.

Tutto sembra pronto e tutto fa pensare che, in un paragone un po' banale, l'autonomia che abbiamo tutti acquisito nell'uso quotidiano del computer per acquistare oggetti o ottenere informazioni, sia perfettamente trasferibile a una metodologia - parallela in parte - di insegnamento. Molti insegnanti difendono il valore di questa metodologia; di questo sono convinti coloro che in questi articoli la propongono, ma la realtà è che attualmente l'autonomia di apprendimento non si è ancora imposta né quasi per niente proposta: prendo ad esempio nella fattispecie la $\mathrm{Fa}-$ coltà di Lettere di Firenze. Il perché non sia apprezzato questo modello è una domanda ancora senza risposta. La mia elementare ipotesi è che in realtà gli studenti sono più pronti degli insegnanti ad affrontare tale metodologia; il cambio di ruolo che impone al docente non è facile da accettare per la perdita di protagonismo che comporta e per lo sforzo che ogni cambiamento implica, ma anche perché il docente deve acquisire un minimo di conoscenze tecniche e sa di doversi aggiornare continuamente se si mette in contatto con le nuove tecnologie.

$\mathrm{Mi}$ auguro che questo libro possa aiutare in questo senso chi ancora non si è deciso a cambiare prospettiva di insegnamento, mettendo realmente l'apprendente al centro del processo di apprendimento. 


\title{
PROJECT WORK TEATRALE
}

\author{
Lucia Alessio \\ Università per gli Stranieri di Siena
}

\section{Introduzione}

In questo articolo prenderò in considerazione il contributo di un project work teatrale all'insegnamento/apprendimento di una lingua straniera. Parlando di "project work teatrale" non intendo riferirmi a generiche attività di drammatizzazione ${ }^{1}$, ma alla realizzazione di un vero e proprio progetto teatrale che, partendo dalla lettura e dalla interpretazione di un dramma, porti alla produzione di uno spettacolo. Il progetto teatrale diviene, in questo processo, un mezzo per permettere agli apprendenti di entrare in contatto con varietà linguistiche e modalità comunicative molto vicine alla interazione reale, di appropriarsi del lessico e delle strutture in modo attivo e infine di utilizzare in modo creativo la lingua di studio. L'autonomia degli apprendenti viene vista in tal senso sia come obiettivo - che coincide con l'obiettivo della didattica in genere - che come strumento di apprendimento.

In qualsiasi processo di insegnamento/apprendimento non si verifica mai un passaggio unidirezionale di conoscenze dall'insegnante ai discenti. C'è sempre una partecipazione attiva degli apprendenti nel costruire il proprio sapere. Ciò si verifica in modo particolare nell'insegnamento/ apprendimento di una L2, dove gli apprendenti padroneggiano già un codice linguistico e possiedono una capacità innata di elaborare attivamente ipotesi sulla lingua di studio. Alla necessità di "trasmettere" conoscenze strutturate nel modo più adatto al livello degli apprendenti, si affianca un processo - in gran parte inconscio e scarsamente governabile - di appropriazione, da parte degli apprendenti, di un nuovo strumento per comunicare e conoscere. Tale appropriazione, secondo l'ipotesi dell'interlingua di Selinker (1972), avviene in modo sistematico, variabile e individuale ed è di fatto difficilmente controllabile.

Se consideriamo il linguaggio come strumento, ci rendiamo immediatamente conto che per impararlo è necessario usarlo: non impariamo a guidare l'automobile solo studiando il codice della strada, a un certo punto bisogna pur salire in macchina e provare a guidare. Imparare ad usare 
il linguaggio significa avere la possibilità di inserire stringhe linguistiche all'interno di un contesto per trasmettere dei significati. Dopo aver appreso funzioni e nozioni linguistiche è dunque necessario che l'apprendente abbia l'occasione di applicarle in un contesto significativo, e a ciò tendono in qualche modo tutti i manuali di lingua che adottano approcci di tipo comunicativo, come molti degli sforzi degli insegnanti nel promuovere giochi, vari tipi di "drammatizzazioni" e discussioni in classe. Purtroppo non sempre questi sforzi sono coronati da successo, molto spesso emergono difficoltà dovute al timore degli apprendenti di esprimersi e mettersi in gioco in una lingua per loro ancora in gran parte estranea. Un modo per superare questa difficoltà è creare in classe un contesto "reale" entro il quale la comunicazione sia una necessità e non solo un esercizio formale. Tale contesto può essere costituito da un project work (cfr. Quartapelle 1999; Ridarelli 1998), dall'uso della L2 per trasmettere contenuti disciplinari (CLIL) o, infine, da un progetto teatrale. Il progetto teatrale gode di un posto privilegiato entro queste attività in quanto, da un lato offre la possibilità, propria di ogni project work di usare la lingua in modo autentico e comunicativo, ponendo l'accento sul significato, dall'altro, essendo incentrato sulla lingua, va a collocarsi nel cuore del processo comunicativo e permette numerose ricadute sia al livello della riflessione metalinguistica che sul piano del contatto tra lingua e cultura.

Wessels (1987:7), noto per essersi occupato dell'applicazione delle tecniche teatrali all'apprendimento delle lingue seconde, individua nei contesti, da lui definiti "scenari", della comunicazione quotidiana una realtà apparente e una realtà di fondo, che non necessariamente coincidono. La realtà apparente include una situazione, un problema e una soluzione. Ad esempio, in una riunione aziendale (situazione) inavvertitamente uno versa un caffè sulla relazione di un collega (problema) e si scusa (soluzione). Ma le parole che proferirà e il tono di queste parole dipenderanno strettamente dalla realtà di fondo, ovvero, dal retroscena, dalle emozioni e dalla progettazione. Diverse saranno le scuse se il collega è un diretto rivale dal quale abbiamo subito svariati soprusi, o se invece si è sinceramente addolorati per aver arrecato un danno a un buon collaboratore e si desiderano ristabilire al più presto ottimi rapporti con lui, $\mathrm{o}$, infine, se la nostra promozione dipende da ciò che il collega dovrà riferire di noi al direttore del personale.

Non c'è corrispondenza biunivoca tra enunciati e atti linguistici: per riprendere un noto esempio di Hymes (1974) l'identico enunciato "ho fame", pronunciato da un mendicante o da un bambino che non vuole andare a dormire, hanno la stessa forma linguistica ma rappresentano atti diversi. Analogamente lo stesso atto direttivo - chiedere a qualcuno di chiudere una finestra - può essere realizzato in molti modi: "per favore potrebbe chiudere la finestra?”, oppure: "fa freddoo!!!!”. Anche se il significato referenziale dei due enunciati è identico, essi veicolano nu- 
merose altre informazioni sulla relazione tra i parlanti, sull'umore di chi le pronuncia, sulla situazione comunicativa ecc. Per tacere dell'ulteriore densità comunicativa conferita loro dalle caratteristiche soprasegmentali, dalla comunicazione cinesica e corporea che, inevitabilmente, accompagnano l'atto dell'enunciazione.

La realtà apparente rappresenta dunque solo la superficie della comunicazione: è la realtà di fondo, il contesto, a fornire a ogni atto linguistico il suo specifico significato. È questa realtà di fondo che mette in moto la motivazione a usare la lingua e a farlo in modo espressivo. Se vogliamo che la lingua degli apprendenti si spinga oltre la mera comunicazione referenziale, finalizzata alla pura e semplice informazione, per dominare una comunicazione complessa, quella in cui siano presenti "l'espressivo, l'ingiuntivo, il poetico, e con essi il non detto, il non verbale ecc." (Lombardi 2004), dobbiamo cercare di rendere la classe un luogo di comunicazione totale. I dialoghi che si trovano nei manuali di lingua non fanno riferimento a questo tipo di contesto: di solito veicolano significati di tipo meramente referenziale, sono basati su relazioni altamente consensuali, sono costruiti per veicolare strutture, funzioni e nozioni linguistiche in modo astratto. In questo tipo di testi l'iniziativa dello studente spesso si limita all'elaborazione di inferenze riguardanti la forma linguistica o la funzione comunicativa presentata. I dialoghi, insieme alle esercitazioni di corredo proposte per fissare le strutture apprese, rispondono alla necessità di fornire "modelli" sui quali in seguito gli studenti dovrebbero poter costruire la propria lingua in modo creativo. Tutti gli insegnanti di lingue hanno però avuto modo di riscontrare la (frustrante) discrepanza tra la lingua dell'esercitazione in classe e la lingua che gli apprendenti usano in interazioni spontanee: il problema è colmare il vuoto tra i modelli proposti e ciò che costituisce la comunicazione "reale". Solo se riusciamo a creare condizioni tali che anche la grammatica riacquisti un valore emotivo, un significato comunicativo, potremo aspettarci un uso maggiormente appropriato della L2 anche nel corso della comunicazione spontanea. Ciò può avvenire creando numerose occasioni per i discenti di usare lo strumento, di cui stanno imparando le regole teoriche, in tutta la sua complessità.

La nostra ipotesi è che ciò possa verificarsi al meglio nell'elaborazione di un progetto teatrale che:

1. mette gli apprendenti in contatto con una lingua contestualizzata più vicina alla realtà della comunicazione quotidiana permettendo loro di sperimentare attivamente le modalità comunicative della L2 attraverso la recitazione teatrale;

2. consente agli apprendenti di sperimentare la propria creatività, di discutere sulle proprie ipotesi linguistiche attraverso un processo di riflessione metalinguistica; 
3. offre l'occasione di lavorare a un progetto comune entro un gruppo sociale che usa la L2 come strumento di comunicazione autentica;

4. instaura un dialogo produttivo tra la cultura di origine e quella della lingua di apprendimento.

\section{La lingua del teatro}

La prima parte del progetto prevede il confronto con testi teatrali. Ai primi livelli di apprendimento si potrebbe iniziare dalla sceneggiatura dei testi presenti nei manuali di lingua (cfr. Holden, 1981, 73, sgg.). Può essere interessante stimolare la classe a produrre situazioni di fondo e a drammatizzare i dialoghi del manuale nella diverse prospettive possibili (per un esempio di contestualizzazione cfr. Appendice 1). Non si tratterebbe più di ripetere in modo passivo stringhe linguistiche apprese, ma di "recitare" una parte nella sua pienezza. E estremamente importante che il contesto comunicativo venga definito in modo particolareggiato: i caratteri, le relazioni tra i personaggi, il retroscena, le emozioni coinvolte, le intenzioni ecc. La definizione dei contesti offre inoltre abbondante materiale di discussione sia nella fase di preparazione delle scene, sia nel commento che può seguire la drammatizzazione. Una volta definita una situazione di comunicazione, è già stato fatto il primo passo verso la realizzazione di una rappresentazione teatrale.

Una seconda, più impegnativa, possibilità è quella di confrontarsi con testi teatrali. La scelta dipenderà dal livello e dagli interessi degli apprendenti e può rivelarsi molto vantaggiosa. Innanzitutto nel testo teatrale è già presente una realtà di fondo, il dialogo è inserito in un contesto significativo, le interazioni raramente sono consensuali: molto spesso il teatro mette in scena i conflitti, l'incomprensione, l'incomunicabilità. Ciò produce inevitabilmente l'irrompere di intenzionalità, emozioni, implicazioni, riferimenti deittici: in pratica tutto ciò che rende la lingua strumento quotidiano di comunicazione. In secondo luogo il testo teatrale, in quanto testo letterario, ha un indubbio valore culturale, agisce da medium con la cultura generale propria della lingua di studio, è altamente motivante e stimolante.

Riteniamo che la lingua del teatro possa costituire quel ponte tra la lingua protetta usata in classe e la lingua che gli apprendenti dovranno usare fuori, del quale tanto si sente la necessità (cfr. Holden 1981:3). Ci rifacciamo a questo proposito all'analisi di Burton (1980) sulle affinità e le differenze tra interazione spontanea (naturally occurring interaction) e interazione scenica (stage interaction). Burton fa notare che sarebbe intollerabile se qualcuno parlasse nella vita reale come se fosse sulla scena, o anche nei termini dei dialoghi di una fiction; altrettanto inaccettabile sarebbe l'introduzione dell'interazione spontanea sulla scena. I tratti tipici dell'inte- 
razione spontanea - false partenze, enunciati poco chiari, sovrapposizioni, elementi paralinguistici involontari - sono assenti dall'interazione scenica dove il dialogo tende invece a essere trasparente e accessibile all'interpretazione dello spettatore. L'attribuzione di realismo ai dialoghi delle fiction e ai testi teatrali è spesso basata su una nozione inadeguata o inaccurata di ciò che è veramente la conversazione spontanea. Questa attribuzione è però possibile in quanto sia l'interazione spontanea che quella scenica rispettano le regole della conversazione, in primo luogo le prime e più fondamentali, individuate dall'analisi conversazionale: l'alternanza e l'avvicendamento dei turni di parola. ${ }^{2}$ Il fatto che il dialogo teatrale si basi sulle stesse regole della conversazione naturale, epurata però dal suo carattere di imprevedibilità ed estemporaneità, fa sì che questo costituisca una versione semplificata della conversazione quotidiana. ${ }^{3}$ D'altra parte il dialogo teatrale rappresenta di per sé anche un testo letterario e ciò ne fa uno dei punti di contatto privilegiati tra lingua e letteratura.

Allo scopo di mostrare praticamente quanto detto, abbiamo preso in considerazione tre testi. Tutti si riferiscono a un invito, a pranzo o a cena. Il primo è tratto da un manuale di lingua italiana per apprendenti tedescofoni (Buongiorno 1, Klett Verlag), il secondo è la trascrizione di una conversazione telefonica registrata, il terzo, un brano di un testo teatrale (Sabato domenica e lunedi di Eduardo De Filippo):

(1)

- Pronto!...Ah, sei tu, Paolo!

- Ti telefono perché stamattina è arrivato un mio collega tedesco. Rimane qui fino a sabato perché dobbiamo risolvere insieme un problema di lavoro molto importante. Però non vorrei andare sempre al ristorante...

- Ma certo, potete venire qui a casa. Però...io purtroppo ho già un impegno per stasera, devo andare a una riunione, mi dispiace.

- Non importa, facciamo così: stasera andiamo al ristorante e domani sera veniamo a casa. Va bene?

- Benissimo! (...)

(2)
C. pronto
L. ciao
C. buonasera $=$
L. = buonasera
C. sto raccogliendo l'insalatina
L. brava [ride] ++ ascolta + volevo chiederti + allora stasera?
C. $\mathrm{xx}$, va tutto ++ tutto regolare $=$

$$
\begin{aligned}
= & \text { ci sia }++. \\
= & x x, \text { allora siamo }+ \text { siamo in } \\
& \text { quattordici }
\end{aligned}
$$


L. oh mio dio + e chi viene ancora?

C. noi + i C $[. .]+$. laaaaa laaa $=$

L.

$$
=\mathrm{O}[\ldots]
$$

C.

$=$ avevo invitato pepxx $\mathrm{O}[\ldots]+$ no tel'avevo detto? =

$$
\text { L. }=\mathrm{eh}
$$

$=$ pepxx non viene

però viene la moglie che + che anche lei è una +una [...]

\section{(3)}

ROSA (...) domani è domenica, teniamo gente a pranzo

PEPPINO Chi viene?

ROSA (aspra) Chi viene?...Viene tua nuora.

PEPPINO Perché è nuora solamente a me?

ROSA Mi ero dimenticata che qua si deve parlare con punto e virgola. (scandendo) "Viene nostra nuora" con Roberto.

PEPPNO (taglia corto) Sì, sì, va bene.

ROSA Ha telefonato lei stamattina: "Mammà, domani è domenica, possiamo venire a pranzo da voi?" Quella quando può evitare di mettersi in cucina a cucinare è tutta felice.

Come si può notare il dialogo (1) è estremamente consensuale e referenziale, nulla fa pensare a forme di disaccordo o a intenzionalità, impliciti, sottintesi, incomprensioni (la consensualità e tanto più sorprendente in quanto si tratta di un rifiuto!). Tutti i dati della "realtà di superficie" sono stati perfettamente esplicitati, ma la realtà di fondo è assente. Il dialogo (2) è estremamente complesso. A parte l'apertura con un "inusuale" doppio saluto (ciao-buonasera-buonasera), un ascoltatore terzo non potrebbe interpretare il significato di sto raccogliendo l'insalatina senza ricorrere a una serie di conoscenze implicite: un precedente accordo per la cena che prevedeva insalata nel menù, il riferimento alla presenza di un orto, proprietà della padrona di casa (C.). Allo stesso modo non si potrebbe comprendere lo stupore di L. se non si sapesse che era già stato stabilito un numero minore di ospiti e che la cena doveva avere un carattere "familiare". Notiamo inoltre tratti caratteristici del parlato: balbettii e frasi incomplete, errori di articolazione, sovrapposizioni, ripetizioni e quant'altro renderebbe improponibile un uso didattico di questo tipo di testo. Il testo (3), al contrario, possiede numerosissimi elementi che potrebbero renderlo di grande interesse per gli apprendenti: l'uso di varietà substandard di italiano regionale (teniamo per abbiamo; mammà per mamma) di forme marcate (è nuora solamente a me), l'uso di metafore (parlare con punto e virgola), e, soprattutto, l'emergere di intenzioni, il definirsi di caratteri, lo svolgersi di un dialogo per nulla consensuale, il costruirsi insomma di quella terza di- 
mensione che, del tutto assente dal dialogo (1) restituisce al linguaggio la sua pienezza comunicativa. ${ }^{4}$

\section{La lingua degli apprendenti}

Il testo teatrale offre dunque un input semplificato, ma non banalizzato. Se proposto come spettacolo teatrale, attraverso spezzoni video per esempio, si rivela un mezzo espressivo di straordinaria potenza e complessità, che permette una più facile decodifica da parte dello spettatore rispetto ad altri tipi di testi letterari. Il sommarsi di diversi codici rende infatti il linguaggio teatrale particolarmente ridondante e quindi "leggibile". Specifici generi teatrali possono essere di facile comprensione in quanto "ipercodificati", ovvero soggetti a convenzioni molto rigide (De Marinis 1983:127-128); un esempio tipico ne è il teatro dei burattini, che per questo si è rivelato uno strumento particolarmente efficace soprattutto ai primi livelli di apprendimento (cfr. Appendice 3).

Ma il progetto teatrale offre anche l'occasione per produrre output, mettendo l'apprendente al centro del suo percorso di apprendimento. Ciò può avvenire nel corso della memorizzazione di un testo teatrale precedentemente scelto, che, durante le prove, può essere recitato più volte, prima con il supporto del testo scritto e poi improvvisando, in modo da mettere ripetutamente a confronto le due versioni (utile è l'uso del registratore). In tal modo l'apprendente è portato a riflettere, sia individualmente che nel gruppo classe, sulle strutture usate.

$\mathrm{Ma}$, nelle attività propedeutiche alla recitazione, saranno gli apprendenti stessi a improvvisare dialoghi autonomamente, partendo da stimoli immaginativi: si può partire dalla sceneggiatura di un testo in prosa, da stimoli visivi o uditivi, da alcune parole/stimolo o, infine, se si tratta di teatro di figura, dalla creazione fisica dei personaggi. ${ }^{5}$ Gli apprendenti avranno l'opportunità di "giocare" con la lingua, di saggiarne le potenzialità, di deviare dalla norma, di confrontarsi con la comicità e l'umorismo, sviluppando quelle capacità che vediamo particolarmente attive nei bambini, e che implicano la presa di coscienza delle regole che governano il sistema della L2. Nel corso di tali attività la componente creativa procederà parallelamente alla riflessione sulla forma linguistica; ne uscirà rafforzata, tra l'altro, l'autostima dell'apprendente, incoraggiato a passare dalla constatazione del "so fare solo questo" al "questo lo so fare solo io" (Huber 2003: 136).

Sia l'improvvisazione creativa, opportunamente guidata, che la ricostruzione creativa di un testo teatrale letterario permettono agli apprendenti di produrre significati che andranno continuamente negoziati nel gruppo classe. Le ricerche hanno mostrato che gli apprendenti spesso rispondono alle mosse di negoziazione - come una richiesta di chiarifi- 
cazione - con una modificazione dell'output. Swain e Lapkin, studiando gli effetti dello sforzo di produrre output comprensibile sull'apprendimento delle L2, hanno riscontrato la correlazione positiva tra negoziazione dei significati e apprendimento (Swain 2005). Un'importante funzione dell'output è quella di dirigere l'attenzione e di innescare i processi di elaborazione. Per esempio l'apprendente potrebbe accorgersi di non riuscire a esprimere con precisione il significato che vorrebbe comunicare, rendendosi consapevole di alcuni dei suoi problemi linguistici. Ciò lo condurrebbe a cercare qualcosa di cui sente il bisogno riguardo alla L2, probabilmente dirigendo l'attenzione all'input rilevante; l'attenzione che ha il potere di innescare i processi cognitivi implicati nell'apprendimento, generando nuova conoscenza linguistica o consolidando quella corrente. Produrre output significa anche mettere alla prova le proprie ipotesi interlinguistiche, che verrano valutate rispetto alle reazioni degli interlocutori. Infine la negoziazione dell'output mette in moto processi di riflessione metalinguistica che produce ipotesi sulla lingua di studio e permette agli apprendenti di progredire nella conoscenza. Lo sforzo creativo fa insomma del discente un ricercatore, lo spinge a realizzare in parole contenuti emozionali che riflettono l'espressione di sé. Anche ai primi livelli di apprendimento è possibile notare lo sforzo di usare la lingua in tutta la sua espressività, come è emerso dall'esperienza di alcuni laboratori di teatro di figura attivati con apprendenti adolescenti (cfr. Appendice 2).

Se vogliamo che la creatività venga ad essere una molla per l'apprendimento, è necessario sia dare importanza all'aspetto linguistico dell'attività, sia a quello "estetico" ed emozionale. Bisogna imparare non solo a parlare, ma a vedere, sentire, pensare, sognare in L2. Solo un coinvolgimento totale permetterà di attivare la motivazione e l'interesse, unirà la conoscenza all'emozione, portando all'apprendimento profondo. Per questo crediamo che l'avere come obiettivo una rappresentazione pubblica possa rappresentare uno stimolo positivo per l'apprendimento. Anche se per alcuni autori nulla può essere più distruttivo di un lavoro sul dramma del sapere che, volenti o nolenti, in una certa data verrà fatta una rappresentazione, crediamo che l'accento sul processo e sul prodotto non siano di per sé antitetici e trovino entrambi spazio adeguato all'interno del project work teatrale.

\section{La lingua nel laboratorio teatrale}

Che cos'è un laboratorio teatrale? È un luogo dove si fanno esercizi di rilassamento, esercizi di immaginazione attiva, dove si lavora sulla fonetica, sulla prosodia, sulla gestualità ecc. Gli strumenti per imparare a gestire queste attività sono ormai numerosi. ${ }^{6}$ E un luogo in cui si lavo- 
ra sostanzialmente in gruppo, si progetta, si prova, ci si sforza insieme di raggiungere un obiettivo comune. La comunicazione in classe non sarà quella monodirezionale insegnante-discenti, ma verrà moltiplicata dallo svolgersi entro un tessuto sociale di relazioni orizzontali tra i partecipanti al progetto in quanto "il primo prodotto del laboratorio teatrale è una microstruttura sociale: il gruppo teatrale, la compagnia" (Guidi, 1988: 29). Il momento del laboratorio offre l'opportunità di usare la lingua in modo autenticamente comunicativo in un contesto finalizzato a uno scopo. L'accento è posto quindi sul significato e sul successo della comunicazione più che sulla sua forma. Nell'attività di laboratorio è il gruppo classe che diviene contesto di scambi sociali, luogo dove si stabiliscono rapporti di socialità a livello di interazione sociale (ruoli sociali) oltre che di scambio comunicativo (flussi di comunicazione). Il ruolo del docente diviene allora quello di "sollecitare flussi di comunicazione, di creare reti di scambi all'interno del gruppo classe" attraverso l'organizzazione dei compiti in un'attività finalizzata a raggiungere un preciso obiettivo (cfr. Vedovelli 2002:116 sgg). Sarà suo compito il cercare di rendere il più possibile fluida la comunicazione e rilassato l'ambiente dove si svolge il laboratorio predisponendo un ambiente sufficientemente sereno e ordinato, dove l'interazione comunicativa sia ben chiara e comprensibile, mettendo a disposizione degli apprendenti "pezzi" di lingua che possano essere usati autonomamente e senza il suo intervento. ${ }^{7}$ Maggiore sarà infatti il flusso di comunicazione, maggiore sarà il successo di questa attività.

Gli scambi comunicativi vedranno gli apprendenti impegnati in azioni comuni finalizzate a raggiungere dei risultati precisi, molti saranno gli argomenti di scambio: dalla valutazione dell'appropriatezza dei testi prodotti, alle osservazioni sulla efficacia della recitazione, alla definizione degli oggetti, degli abbigliamenti e delle scene, dei movimenti, delle luci, delle musiche ecc. Avranno a che fare con istruzioni e con scambi di opinioni con l'insegnante e i compagni.

Riteniamo che con principianti assoluti sia utile, in questa fase, ricorrere al language alternation proposta da Curran (cfr. D Prete 1998: 48-50). L'insegnante potrebbe, cioè, lasciare che gli studenti si esprimano nella loro lingua madre o in una lingua comune a entrambi e tradurre ogni volta le frasi dette nella L2. Allo stesso modo, ogni volta che l'insegnante si rivolge a uno o più studenti userà prima la L2, poi, se necessario, tradurrà la frase nella L1 degli apprendenti o in una lingua compresa da tutti. Ciò impedisce il crearsi di blocchi emotivi e, rispettando i tempi degli apprendenti, permette loro di superare con maggiore facilità i propri timori nell'esprimersi in L2. Riteniamo tuttavia che l'insegnante debba predisporre l'ambiente nel modo più idoneo per incoraggiare il passaggio dalla L1 alla L2. Ciò può avvenire mettendo delle etichette sugli oggetti di uso più comune, in modo da 
permetterne la immediata identificazione, incoraggiando gli studenti a rendersi autonomi attraverso la costruzione di schede di riferimento nelle descrizioni e discussioni, preparando accuratamente istruzioni chiare e comprensibili per ogni fase del lavoro.

\section{L'aspetto interculturale}

Secondo il Consiglio d'Europa (Council of Europe, 2001: 1) "Communication calls upon the whole human being"; tra i communicative needs dell'apprendente, cui la didattica dell'italiano L2 dovrebbe rispondere, c'è il bisogno "to achieve a wider and deeper understanding of the way of life and forms of thought of other peoples and of their cultural heritage" (Council of Europe, 2001: 3). La competenza interculturale chiama in causa l'essere umano nella sua totalità e trasforma l'apprendimento di una L2 in un'esperienza di vita. Per far questo è indispensabile venire a contatto con forme di cultura proprie della L2 e con quella complessa rete comportamentale che avvolge, spesso inconsapevolmente, le nostre azioni in ogni momento. Nel lavorare insieme è più facile che tali componenti vengano in primo piano. Il progetto teatrale mette gli apprendenti a confronto con l'universo culturale e linguistico della L2, non in modo impersonale, come potrebbe avvenire nella semplice fruizione di un testo letterario o spettacolare, ma chiedendo loro di parlare, agire e sentire in lingua straniera. Si verifica in tal modo quel passaggio tra sapere, saper fare e saper essere auspicato dal Consiglio di Europa come obiettivo primario della didattica delle lingue. Confrontando i propri codici comportamentali con quelli usati nella cultura della L2 si innesca una riflessione che porta alla scoperta dell'alterità e allo sviluppo della consapevolezza interculturale favorendo la motivazione e l'autonomia dell'apprendimento. Il fatto che ciò avvenga all'interno di un gruppo impegnato in un lavoro comune, consente lo svolgersi di tale confronto in un clima di collaborazione costruttiva e di reciproco rispetto.

Le esperienze in questo senso hanno messo in rilievo l'importanza di gestire consapevolmente il volume della voce, con conseguenze visibili sull'efficacia della comunicazione (è noto che nei paesi nord-europei si parla con un volume più basso di quello usato nei paesi mediterranei $\mathrm{e}$ che ciò genere tutta una serie di inferenze erronee); ancora è utile lavorare sui turni di parola, sulla gestualità, sulla gestione dello spazio e del tempo, sulle pause, sul ritmo intonativo, sugli aspetti prosodici, sui rapporti gerarchici, ecc. (cfr. Balboni 1999). 
Appendice 1

Esempio di contestualizzazione di un dialogo tratto da Rete 1 della casa editrice Guerra:

Sandro: Finalmente in Italia! Finalmente a Roma!

Maria: Come sono stanca!

Sandro: Anch'io. E ora c'è il controllo dei passaporti.

Agente di polizia: Passaporto per favore. Lei si chiama Maria Caballero. Perché è in Italia?

Maria: Studio italiano a Siena.

Agente: Quando torna in Argentina?

Maria: Alla fine del corso.

Agente: Tutto bene. Un altro per favore.

Realtà apparente: due giovani (come si deduce dalla foto di accompagnamento al testo) arrivano stanchi all'aeroporto, la ragazza è argentina, del ragazzo non conosciamo la nazionalità; c'è il controllo dei passaporti; l'agente fa delle domande a cui la ragazza risponde in modo appropriato. Proviamo ad aggiungere degli elementi di fondo al dialogo. Supponiamo che Sandro sia italiano e Maria sia la sua ragazza argentina venuta a studiare italiano a Siena per poter fare la conoscenza della famiglia del fidanzato. Forse Maria non si è sentita di fare il viaggio da sola e ha convinto Sandro ad accompagnarla. Sandro è irritato del lungo ed estenuante viaggio, non ancora terminato (devono ancora arrivare a Siena), ed è pentito della decisione presa. In questo caso quando lei dice: "come sono stanca!" lui risponde "anch'io" con un tono che vuole accusarla di essere poco autonoma e indipendente. Anche le parole pronunciate da Sandro: "E ora c'è il controllo dei passaporti" acquistano un significato non neutrale, e rivelano un pensiero traducibile come: "insomma, mi tocca anche questa!”.

\section{Appendice 2}

Presentiamo due brevi produzioni di studenti adolescenti tedescofoni nel laboratorio di burattini. Gli studenti, che avevano alle spalle circa 10 ore di lezione frontale, avevano ricevuto delle schede con alcune espressioni tipiche per esprimere i sentimenti. Avevano poi ricevuto degli "scenari", delle brevi tracce su cui costruire un dialogo con l'aiuto dell'insegnante. In seguito hanno cercato di recitare le parti muovendo i burattini: 
Dramma della gelosia

Piromanillo: Ciccina! Dove sei?

Ciccina: Ahi! Cielo e mio merito Piromanielo! E molto cattivo! Aiuto!

Beduino: Non posso crederci! Santo cielo! Che grossa pancia!

Piromanillo. Attenzione, sono rabbioso. Ciccina è mia!

Emergenza

Carabiniere: Buongiorno!

Pulcinella: Heulen!

Carabiniere: Che succede?

Pulcinella: Un cane mi ha morso!

Carabiniere: Dio mio! Non è possibile!

Appendice 3

Descriveremo una breve esperienza che ha avuto come protagoniste 4 studentesse di livello A1/A2 del programma estivo 2007 della Vanderbilt University. Si è trattato di un numero molto ridotto di incontri: 5 incontri di 2 ore ciascuno e pertanto l'esperienza deve essere considerata come molto provvisoria. Tuttavia ci sembra possa essere interessante soprattutto in direzione dell'elaborazione di una procedura per l'uso del teatro nell'insegnamento delle L2.

Presentazione: è stata fatta una presentazione in inglese con power point rivolta a tutti gli studenti (circa 30) al fine di spiegare il contenuto del laboratorio. Alla fine della presentazione si sono iscritte le 4 studentesse che poi hanno seguito il laboratorio.

Primo incontro:

- Iniziamo col rilassamento e gli esercizi di respirazione, come faremo anche in tutti gli altri incontri. Siccome tre delle 4 studentesse sono principianti assolute, le istruzioni vengono date prima in italiano e poi in inglese (per es. chiudete gli occhi - close your eyes - e trovate una posizione comoda - find a relaxed position ...). A seguito degli esercizi di respirazione vengono proposti alcuni esercizi di vocalizzazioni dove si richiede di esagerare molto la posizione delle labbra (una delle maggiori difficoltà degli anglofoni, infatti, è l'identificazione dei suoni vocalici italiani che non corrispondono a quelli della loro lingua madre).

- Per creare un'atmosfera positiva nel gruppo viene proposto un esercizio classico, quello della "Camminata". Si tratta di camminare per 
la stanza (appropriazione dello spazio) accelerando il passo o meno (consapevolezza corporea) e salutando poi gli altri partecipanti in vari modi: formale, informale, dandosi la mano, esprimendo sentimenti di sorpresa, ostilità, ecc (appropriazione dello spazio sociale). L'insegnante partecipa all'attività.

- Animazione oggetti: vengono distribuiti alcuni oggetti presenti in classe (un libro, una penna ecc.) Ognuno deve poggiare a terra il proprio oggetto e poi riprenderlo come se fosse qualcosa di molto delicato e importante, qualcosa molto amato. L'oggetto viene "cullato" e poi passato al compagno nominandolo con un tono che indichi il nostro sentimento nei suoi confronti. Il compagno ringrazia esprimendo identico sentimento. Facciamo lo stesso considerando gli oggetti volta a volta qualcosa di pericoloso, schifoso ecc.

- Ognuno attribuisce un'identità al proprio oggetto e la descrive agli altri con l'aiuto di una scheda di lavoro.

Secondo incontro:

- Rifacciamo il rilassamento come la prima volta, ma questa volta solo in italiano. Dopo la vocalizzazione vengono introdotti per la prima volta gruppi sillabici (/ka/ / ko/ / ku/ / ki// ke/; /ba/ /bo/ /bu/ /bi/ /be/; ecc.) e poi le consonanti geminate pronunciate ritmicamente (/ $\mathrm{ba} / / \mathrm{ba} /$ - /ba/ /ba/ - /babba//babba//babba/ /babba/;/ma// $\mathrm{ma} /$ - /ma/ /ma/ - /mamma/ /mamma/ /mamma/ /mamma/; ecc.)

- Costruzione del personaggio: riprendiamo le schede dei personaggi fatte la volta precedente e continuiamo la presentazione. A ogni personaggio viene attribuito un carattere e una voce.

- Le studentesse visionano dei videoclip di burattini dove si mettono in luce alcune caratteristiche specifiche di questo genere di spettacolo: il carattere universale (burattini iraniani), la presenza di canto, ritmo, la presenza di un eroe e di un antagonista (guarattelle napoletane), il movimento della mano (burattini cinesi).

- Le studentesse ricevono una scheda per la costruzione della storia che presenta alcune funzioni universali come quelle individuate da Propp per la fiaba. Leggiamo insieme la scheda. A casa hanno il compito di discutere e decidere, con l'aiuto delle schede, la storia da rappresentare, partendo dai personaggi nati nel corso della drammatizzazione di oggetti e tenendo in considerazione gli elementi individuati nei videoclip.

Terzo incontro:

- Le studentesse iniziano a raccontare la storia che hanno pensato insieme. La storia viene esposta prevalentemente dalla studentessa che ha una maggiore competenza in italiano, ma le altre partecipano attivamente. I termini sconosciuti vengono scritti sulla lavagna. 
- Il rilassamento avviene questa volta dopo la narrazione della storia, anche perché una studentessa arriva in ritardo e decidiamo di aspettarla. Dopo la vocalizzazione le studentesse hanno il compito di ripetere, con un solo respiro, una frase inizialmente breve che poi si allunga sempre più attraverso espansioni (sono andata al cinema; sono andata al cinema con bianca; sono andata al cinema con la mia amica bianca; sono andata al cinema con la mia amica bianca a vedere un film ecc.)

- Diciamo la frase con toni diversi.

- Cominciamo a far parlare i personaggi della storia. Ognuno si presenta con la propria voce. Ci esercitiamo con la voce a ripetere le presentazioni.

- Allestiamo il laboratorio per costruire le figure. Ci sono molti materiali ognuno con una etichetta: forbici, colla, carta, pennarelli ecc. Vengono distribuiti sul tavolo e le studentesse devono memorizzare $\mathrm{i}$ nomi per 3 minuti. A turno ripetono i nomi memorizzati.

- L'insegnante fornisce istruzioni verbali per la costruzione del burattino (di carta). Le studentesse eseguono. Se non è necessario non vengono date dimostrazioni pratiche delle attività richieste in modo da esercitare la loro abilità di comprensione. Vengono introdotte e fissate alcune forme che loro potrebbero utilizzare nella comunicazione come: ho bisogno di, mi dai.., vorrei..., ecc.

Quarto incontro:

- Rilassamento e respirazione. Vocalizzazioni. Le studentesse devono ripetere una filastrocca prima a bassa voce poi a volume sempre più alto.

- Sulla base della storia che le studentesse hanno raccontato, cerchiamo di improvvisare dei dialoghi che scriviamo. Purtroppo non c'era il tempo per fare il lavoro di registrazione e autocorrezione dei dialoghi e alla fine il testo definitivo è stato scritto dall'insegnante che lo ha spedito alle studentesse via mail in modo che potessero cominciare a studiarlo durante la settimana.

- Cominciamo a provare le varie scene sia a coppie che tutti insieme.

Quinto incontro

- Rilassamento.

- Prove di recitazione nel teatro.

- Spettacolo di fronte ai compagni che arrivano nell'ultima mezz'ora.

- Rinfresco.

\section{Bibliografia}

Alessio L., Sgaglione A., 2007, Invito a teatro. Insegnare l'italiano a stranieri attraverso testi teatrali italiani, Edilingua, Atene. 
Almond M., 2005, A Complete Drama Approach To English Language Teaching, Modern English Publishing.

Cangià C., 1992, L'altra glottodidattica. Bambini e lingua straniera fra teatro e computer, Giunti, Firenze.

Comodi A., Gaone R, 2001, L'italiano si impara a teatro, Società Sifa, Perugina.

Holden, S., 1981, Drama in Language Teaching, Longman, Essex .

Huber R., 2003, Im Haus der Sprache wohnen. Wahrnehmung und Theater im Fremdsprachenunterricht, Niemeyer, Tübingen.

Maley A., Duff A., 1983 (I ed. 1978), Drama Techniques in Language Learning, Cambridge Univeristy Press, Cambridge.

Marini Maio N., 2004, “I sei personaggi siamo noi”, Italica 81, 4, Winter 2004.

Quartapelle F. (a cura di), 1999, Didattica per progetti, Franco Angeli, Milano.

Ridarelli G., 1998, "Project work", in C. Serra Borneto, C'era una volta il metodo, Carocci, Roma, pp.173-187.

Schewe M. L., 1993, Fremdsprache inszenieren. Zur Fundierung einer drama-pädagogischen Lehr-und Lernpraxis, Zentrum für pädagogische Berufspraxis, Universität Oldenburg, Oldenburg.

Schewe M. L., Shaw P., 1993, Towards drama as a method in a foreign language classroom, Peter Lang, Frankfurt /M.

Swain, M., 2005, “The output hypothesis: Theory and research”, in E. Hinkel (Ed.), Handbook on research in second language teaching and learning, Erlbaum, Mahwah (NJ), pp. 471-484..

Wessels C., 1987, Drama, Oxford University Press, Oxford.

Note

${ }^{1}$ Numerosissime sono le attività che possono essere comprese sotto la definizione di "drammatizzazione": creative dramatics (Sam 1990); creative drama (Dodge 1998); strategic interaction (Di Pietro 1987); drama activities (Dougill 1994); drama techniques o drama (Chauhan 2004; Maley/Duff 1996); Drama in Education for Language Learning (Healy 2004); dramapädagogische Lehr- und Lernpraxis (Schewe 1993); Drama Approach (Almond 2005). Tutte queste espressioni si riferiscono ad attività che vanno dalle attività drammatiche in senso proprio (drama) (come role-play, simulazioni, scenario enactment, mimo, canzoni), fino alla scrittura e/o messa in scena di commedie o sketches. Sebbene molte vengano utilizzate nelle attività teatrali, sono comunque da distinguersi dal teatro vero e proprio (theatre) per l'accento posto sul processo più che sul prodotto. L'obiettivo dell'attività è nel caso delle attività drammatiche quello di esercitare la lingua in classe; nel caso del teatro anche il realizzare uno spettacolo per un pubblico.

2 "1) il cambiamento dei parlanti si ripete più volte, o comunque avviene almeno una volta; 2) in genere, si parla uno alla volta” (Sacks, Schegloff, Jefferson 2000 (1974): 100).

${ }^{3}$ Burton ritiene i testi drammatici in generale e quelli moderni in particolare una risorsa estremamente ricca per l'analisi conversazionale, interessata a descrivere le strutture e i vincoli sociolinguistici che regolano la conversazione naturale.

${ }^{4}$ Per un esempio di didattizzazione del brano cfr. Alessio, Sgaglione 2006; per esperienze di allestimento di progetti teatrali in lingua italiana cfr. Nicoletta Marini Maio 2004; Comodi, Gaone 2001; "Uno spettacolo teatrale per imparare l'italiano" in Tendenze Italiane, $\mathrm{N}^{\circ} 15$. 
${ }^{5}$ Alcune interessanti proposte in questa direzione possono trovarsi in Huber 2003; Holden 1981; Maley, Duff 1983; esempi di uso creativo della lingua italiana in Zamponi 1986 e nel noto saggio di G. Rodari, Grammatica della fantasia (1993). Per un esempio di attività a partire dalla creazione dei personaggi cfr. Appendice 3 .

${ }^{6}$ Per esercizi di respirazione e prosodia: Wessels 1987: 62-74; Hardison, Sonchaeng 2005; esercizi di riscaldamento per la formazione del gruppo: Maley, Duff 1983: 38-86.

${ }^{7}$ Un interessante metodo per rendere autonomi gli studenti ed alleggerire il compito dell'insegnante nel momento del lavoro in classe, soprattutto se si intendono intraprendere attività di carattere pratico come costruire scenografie, oggetti, costumi o figure nel teatro di figura è quello dello Stationenlernen descritto in Fremdsprache Deutsch, 35,2006 . Un'altra interessantissima, quanto impegnativa, proposta è quella dell'uso del computer in classe fatta dalla Cangià (1992). 


\title{
"FIRENZE COM'ERA". PROGETTO PER L'APPRENDIMENTO AUTONOMO DELLA LINGUA ITALIANA
}

\author{
Grazia Giannelli, Caterina Sani
}

New York University in Florence

\section{Introduzione}

Il nostro contributo consiste nella presentazione del progetto "Firenze com'era", un progetto per l'apprendimento della lingua italiana, realizzato in due classi di italiano di un corso intermedio intensivo (il cui livello può essere paragonato a un $\mathrm{B} 1$ con qualche elemento $\mathrm{B} 2$ secondo il quadro comune di riferimento europeo) della New York University in Florence durante il semestre primaverile 2007.

Con questo progetto si è cercato di mettere in pratica il concetto di "autonomia nell'apprendimento della lingua" che ha acquistato una grande rilevanza nel panorama universitario italiano e internazionale, attraverso la realizzazione di strumenti didattici che permettono allo studente di "mobilitare e sviluppare la sua capacità di apprendere in modo attivo così che possa imparare ad imparare in modo autonomo" (Evangelisti-Argondizzo, 2002).

L'obiettivo di migliorare la qualità dell'apprendimento nei corsi di lingua straniera è stato quindi perseguito nella prospettiva ben sintetizzata da Little: "Effective communication depends on a complex of procedural skills that develop only through use and if language learning depends crucially on language use, learners who enjoy a high degree of social autonomy in their learning environment should find it easier than otherwise to master the full range of discourse roles on which effective spontaneous communication depends" (Little, 2003).

Nel nostro lavoro, dopo una presentazione dei principali contributi teorico metodologici sul tema e sui fattori di criticità dell'approccio, verranno descritti gli aspetti più importanti dell'applicazione del project work ai nostri corsi.

\section{Inquadramento teorico-metodologico}

L'idea di sviluppare un progetto da affiancare alle lezioni di classe è nata dalla necessità di trovare uno strumento didattico che permettesse 
di rispondere a due esigenze principali: quella di migliorare l'apprendimento della lingua italiana in un quadro anche di acquisizione di conoscenze sulla società e sulla cultura italiane; e quella di individuare e sperimentare nuovi metodi in grado di stimolare l'interesse degli studenti anche permettendo loro di utilizzare la lingua come strumento di comunicazione in un contesto autentico.

Come tradurre nella pratica questi bisogni quando i tempi e i contenuti dei corsi sono già prestabiliti da un sillabo di dipartimento? La risposta alle nostre esigenze è stata quella di proporre agli studenti di sviluppare un progetto: "esplorativo o d'ambiente" che "presuppone un contatto con parlanti nativi in un ambiente in cui la lingua oggetto di studio rappresenta la lingua della comunicazione" (Ciliberti, 1994).

Il contesto di riferimento in cui si colloca questo tipo di approccio è quello del project work. L'interesse verso i project work e la loro integrazione nello studio della seconda lingua è un argomento molto studiato, (si veda Carter e Thomas (1986), Ferragatti e Carminati (1984), FriedBooth (1982, 1986), Haines (1989), Legutke (1984, 1985), Papandreou (1994), Sheppard e Stoller (1995), e Ward (1988) e Stoller (1997)). È quindi ormai sperimentato che organizzare progetti è un modo per insegnare lingua e contenuto nello stesso tempo. Gli studenti sviluppano le loro abilità linguistiche e diventano responsabili del loro apprendimento."By integrating project work into content-based classrooms, educators create vibrant learning environments that require active student involvement, stimulate higher level thinking skills, and give students responsibility for their own learning" (Stoller 1997). Fried-Booth scrive che l'insegnamento della lingua tramite un progetto crea "un ponte" tra l'uso della lingua straniera in classe e l'uso della lingua nelle situazioni reali fuori dalla classe. Ciò avviene "by placing learners in situations that require authentic use of language in order to communicate - e.g. being part of a team or interviewing others (Fried-Booth, 1997)".

Come altri approcci formativi il project work è finalizzato a enfatizzare gli aspetti di coinvolgimento degli studenti, la cooperazione e la loro responsabilizzazione, ma ha come elemento caratterizzante quello di richiedere agli studenti di lavorare insieme per un lungo periodo di tempo, sia in classe sia fuori, in collaborazione con parlanti nativi, il tutto finalizzato alla realizzazione di un prodotto finale da presentare a un pubblico.

Più specificamente il project work è caratterizzato dai seguenti elementi distintivi (Stoller, 1997):

- il miglioramento della propria capacità linguistica e culturale avviene sviluppando un tema della vita reale piuttosto che operando su "specific language targets";

- il lavoro è svolto prevalentemente dagli studenti, l'intervento dell'insegnante è solamente quello di offrire supporto e guida nelle diverse fasi del progetto; (nel nostro caso, invece, come si vedrà in seguito, 
l'insegnante ha avuto un ruolo maggiore nella prima e seconda fase del progetto, mentre successivamente il suo intervento si è ridotto notevolmente);

- il lavoro ha finalità di cooperazione e non di competizione. Gli studenti possono lavorare singolarmente, in piccoli gruppi o tutta la classe nel suo insieme scambiandosi le idee, le informazioni e dividendosi i compiti;

- la realizzazione del progetto richiede l'integrazione di diverse "abilità" e di diverse fonti informative, riproducendo così i processi della vita reale;

- la realizzazione di un prodotto finale dà al progetto una motivazione reale. Naturalmente il valore del progetto non sta nel prodotto finito, ma nel processo attraverso cui si è avuta la sua realizzazione;

- un project work è potenzialmente più motivante e stimolante delle lezioni di classe. Esso dà allo studente fiducia e autonomia, così come migliora le abilità linguistiche, cognitive e culturali.

L'applicazione dello strumento del project work ha però incontrato anche varie difficoltà, rinvenibile anche nella letteratura. Da alcuni studi di valutazione sui project work utilizzati in classi di inglese come seconda lingua (ESL), a esempio, sono emersi risultati poco soddisfacenti in termini di apprezzamento da parte degli studenti. In particolar modo essi ritenevano che questa attività li distraesse da ciò che consideravano più importante, come lo studio della grammatica e del vocabolario, e non riuscivano a capire i benefici derivanti da questo approccio, (Beckett, 2002). Per il superamento di questi problemi appare di estrema importanza il processo di preparazione degli studenti affinché siano consapevoli dei vantaggi e delle opportunità offerte da questo metodo, nonché dei contenuti specifici del lavoro da svolgere. "Incorporating project work in more traditional classroom requires careful orchestration and planning" (Fried-Booth, 1986 e Haines, 1989).

Alcuni autori hanno suggerito strumenti per superare le difficoltà sopra ricordate. In particolare Beckett e Slater (2005) propongono il "Project Framework", un sistema che si articola in un "planning graphic" e in un "project diary", studiato per aiutare l'insegnante e gli studenti a insegnare/imparare simultaneamente lingua e contenuto.

Un altro aspetto critico del project work, che ha richiesto una specifica riflessione, è costituito da quanta autonomia dare agli studenti e quanto/come/quando devono/possono intervenire gli insegnanti. Diventare autonomi, infatti, "è un processo graduale di indipendenza che va preceduto da fasi di dipendenza formativa all'interno delle quali il formatore può solo facilitare il percorso verso l'autonomia" (Liliana Landolfi, 2002). Anche in questo caso appare molto importante la fase preparatoria al lavoro vero e proprio, sia per rendere più consapevole l'autonomia 
dello studente, sia per consentire all'insegnante di comprendere il livello di intervento richiesto dalla specifica classe.

Nel nostro caso applicativo è stato seguito un approccio dinamico che partendo dagli strumenti descritti permettesse di adattarsi via via alle diverse realtà studentesche considerate. Le soluzioni adottate saranno poi approfondite nella descrizione delle singole fasi del progetto.

\section{Il project work}

Per ottenere l'obiettivo prefissato di favorire l'apprendimento della lingua italiana insieme a quello dei principali caratteri socio-culturali della realtà fiorentina, l'idea base è stata quella di impostare il lavoro in una prospettiva diacronica che consentisse agli studenti il contatto con un periodo storico e nello stesso tempo con la realtà di oggi. Il tema scelto è stato l'analisi della società fiorentina in due distinti periodi: quello attuale e quello degli anni '30 - '40. Questa soluzione ci ha permesso infatti di combinare gli aspetti di interazione con il mondo di oggi con quelli culturali legati alla storia di Firenze e alla sua letteratura. Su questa traccia è stata definita una prima fase di preparazione al project work articolata in un ciclo di lezioni in cui sono stati forniti gli strumenti e i materiali di base per comprendere il contesto socio-culturale locale. Tenuto conto del livello delle classi, abbiamo pensato a un argomento che potesse

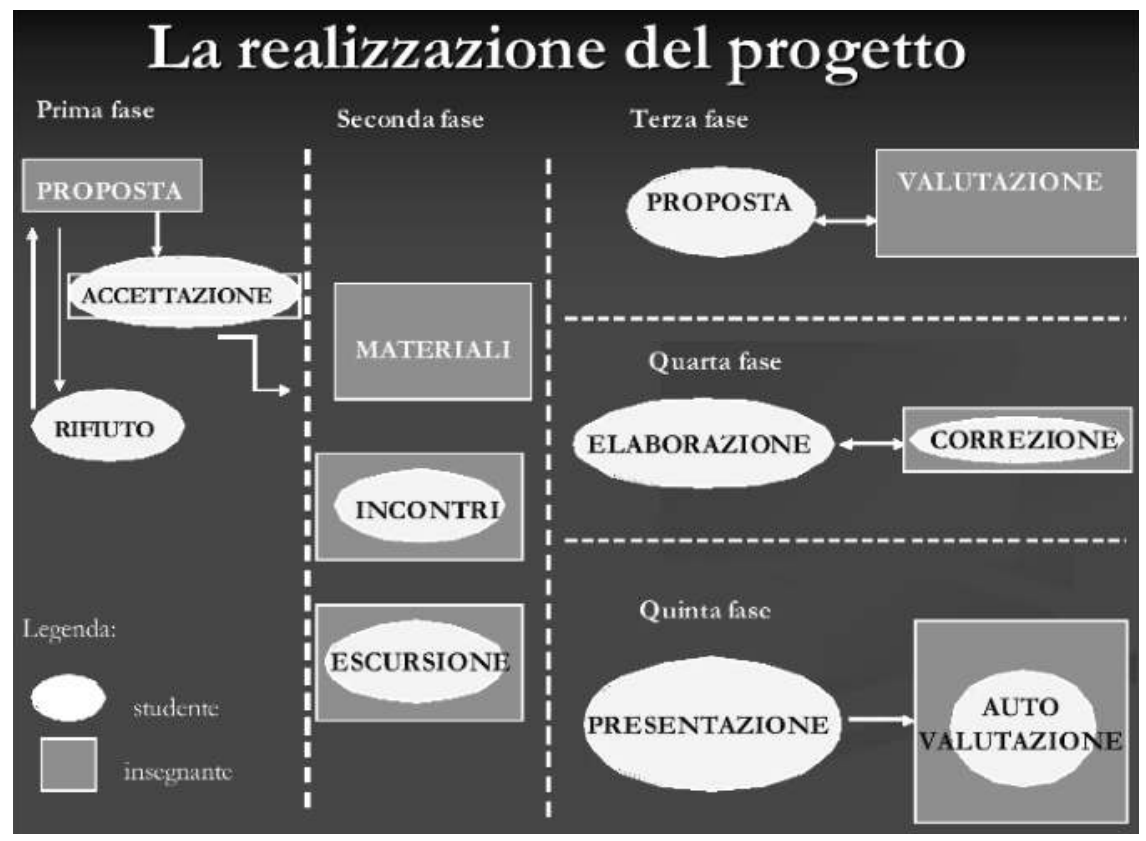


essere interessante per gli studenti - non troppo difficile e comunque in grado di stimolare l'interesse per tutta la durata del progetto - che permettesse di studiare qualcosa di nuovo in italiano in un contesto reale, che stimolasse la creatività e l'immaginazione, e che esponesse gli studenti a una varietà di risorse da poter permettere loro di migliorare la loro abilità nell'uso della lingua venendo a contatto con la cultura locale. Si è così pervenuti alla definizione di una proposta vera e propria di project work che è stata quindi sottoposta agli studenti: "Firenze com'era. Un confronto tra passato e presente".

Nello schema (Fig. 1) che segue, abbiamo suddiviso in cinque fasi logiche la realizzazione del nostro progetto: condivisione della proposta di project work; preparazione della classe con lezioni e distribuzione di materiali; valutazione e aggiustamento della bozza di realizzazione del progetto presentata dagli studenti; realizzazione del lavoro ed eventuali correzioni; presentazione del lavoro e autovalutazione.

\subsection{Prima fase: condivisione della proposta di project work}

La prima fase, particolarmente delicata, è rappresentata dalla proposta del progetto agli studenti. È molto importante, secondo noi, che in questo momento l'insegnante spieghi i vantaggi che derivano dalla realizzazione del progetto, che sono:

- lingua usata come strumento di comunicazione in un contesto autentico;

- possibilità di combinare lingua e contenuto: si sviluppano le abilità linguistiche insieme all'approfondimento culturale, in quanto gli studenti, sulla base di materiali letterari e documentari, interagiscono con la cultura dell'ambiente facendo domande, ascoltando, leggendo e scrivendo;

- responsabilizzazione dello studente: lavorando al di fuori delle lezioni di classe si favorisce l'apprendimento autonomo, cioè lo studente diventa responsabile del proprio apprendimento;

- gli studenti imparano a collaborare in "a co-operative classroom" (Legutke \& Thomas, 1991) per raggiungere un obiettivo comune: un prodotto concreto in lingua italiana. Il valore del progetto non sta comunque nel prodotto finito, ma nel processo attraverso il quale si è giunti alla sua realizzazione;

- stimolare l'interesse all'apprendimento della lingua: lavorare per raggiungere uno scopo è anche un modo "motivato" di studiare una lingua straniera, lo studente è più stimolato a sviluppare le proprie conoscenze linguistiche.

Una volta presentati i vantaggi, abbiamo proposto agli studenti il tema del progetto: "Firenze com'era . Un confronto tra passato e presente" e 
spiegato che consisteva nella realizzazione di un prodotto a scelta degli studenti, sia nella forma che nei contenuti, che mostrasse il confronto tra la Firenze degli anni '30 - '40 e la Firenze di oggi.

Abbiamo lasciato libertà alle classi di accettarlo o rifiutarlo, perché secondo noi è importante la motivazione dello studente. L'insegnante deve mettere in chiaro che il progetto è un qualcosa in più rispetto al corso e pertanto deve essere portato avanti separatamente dalle lezioni di classe. L'impegno richiesto alla classe è quindi elevato e solamente un gruppo altamente motivato può trarne tutti i benefici del caso. Lavorare a un progetto è "a detailed study of a subject" che richiede "a lot of time and effort" (Collins Cobuild, 2001).

Abbiamo inoltre spiegato che lo sviluppo di un progetto può essere svolto nel modo migliore se il lavoro viene portato avanti con la collaborazione di tutti gli studenti, così che laddove uno studente è più debole può essere aiutato, così come studenti non abituati a lavorare in modo autonomo possono non essere in grado di organizzare la loro attività e siano facilitati "by planning cooperative, small group work beforehand" (Fried-Booth, 1986 e Haines, 1989).

Nel nostro semestre primaverile 2007, il progetto è stato proposto a tre classi, ma solamente due hanno accettato di farlo.

\subsection{Seconda fase: preparazione della classe con lezioni e distribuzione di materiali}

Accettato il progetto, è iniziata la seconda fase. È questa una fase in cui l'insegnante ha un ruolo decisivo, in quanto deve inquadrare l'obiettivo del progetto, mettere a disposizione dello studente il materiale di base, supportare eventuali ricerche dirette per completare le informazioni necessarie, in modo da permettergli di portare avanti in autonomia la realizzazione del progetto.

È anzitutto importante che agli studenti sia fornito un vocabolario di supporto così che il progetto "operates within the student language learning capacity " (Fried-Booth, 1982). Nel nostro caso il materiale principale era costituito da testi e fotografie d'epoca. Il testo che abbiamo proposto è stato Il Quartiere di Vasco Pratolini, che ci è sembrato adeguato per consentire di familiarizzare con un vocabolario e una cultura d'epoca. Sono state lette alcune pagine in classe che sono state analizzate sia da un punto di vista linguistico sia culturale. Si è fatta attenzione al vocabolario usato e sono stati evidenziati alcuni aspetti della vita a Firenze negli anni '30 - '40:

- com'erano le strade;

- com'erano le abitazioni;

- quali erano i negozi;

- quali erano i mestieri;

- cosa si mangiava; 
- quali mezzi di trasporto si usavano;

- cosa si faceva nel tempo libero;

- come si svolgevano le feste tradizionali.

Alcuni capitoli sono stati poi assegnati come compito da svolgersi al di fuori della classe. Per accertarsi che tutti gli studenti avessero assimilato il materiale offerto, abbiamo monitorato l'apprendimento linguistico/ culturale degli studenti con verifiche scritte e orali inserite nella scansione proposta dal sillabo.

Dopo avere controllato l'apprendimento del vocabolario e della cultura, il ruolo attivo dell'insegnante si è ridotto. È importante che gli studenti si sentano responsabili del loro lavoro e partecipino in modo autonomo alla sua realizzazione. L'insegnante è comunque sempre a disposizione e assume il ruolo di supervisore nei diversi stadi di avanzamento del lavoro aiutando gli studenti ogni qualvolta venga richiesto. Solo così facendo gli studenti si possono immergere nel progetto, sentirsi motivati e sviluppare un'autonomia nell'apprendimento.

Una volta acquisito il materiale proposto, gli studenti infatti hanno continuato in modo autonomo la raccolta di informazioni. Gli studenti si sono recati nel quartiere di Santa Croce, hanno ripercorso le strade citate nel testo "Il Quartiere" e hanno predisposto con l'insegnante un questionario per raccogliere informazioni sulle differenze tra il quartiere oggi e il quartiere di Pratolini. Il questionario è stato quindi somministrato a commercianti e residenti della zona da parte degli stessi studenti.

Successivamente sono stati organizzati degli incontri con volontari in pensione del quartiere di Santa Croce, al fine di permettere agli studenti di avere un paio di lezioni a disposizione per parlare direttamente con persone vissute in quella zona di Firenze tra gli anni '30 -'40. Gli studenti hanno approfittato di questa occasione per porre domande utili al loro progetto. Hanno preparato infatti un ulteriore questionario in autonomia, per raccogliere le informazioni mancanti e sciogliere gli ultimi dubbi sul lavoro. I volontari, avvertiti dalle insegnanti dell'argomento sul quale sarebbero stati intervistati, hanno portato in classe foto d'epoca e hanno arricchito le interviste degli studenti raccontando aneddoti e curiosità sulla loro infanzia e giovinezza.

Un altro incontro è stato organizzato con ragazzi dell'Università degli studi di Firenze e con una classe dell'ultimo anno di un liceo. In queste occasioni i nostri studenti hanno condotto conversazioni in modo sciolto e spontaneo su temi riguardanti i giovani e su argomenti d'attualità. In questo modo i nostri studenti sono venuti a conoscenza di un altro punto di vista sulla realtà fiorentina, probabilmente più vicino al loro tempo, e di un gergo giovanile che è stato poi utilizzato anche nel progetto.

Gli incontri sono stati un momento di grande "soddisfazione" da parte degli studenti che si sono dimostrati capaci di condurre una con- 
versazione con un grado di scioltezza e spontaneità con parlanti nativi in completa autonomia. Sembra che effettivamente si sia realizzata "an integration of language skills (Eyring, 1997) and use meaningfully for communicating, conceptualising, inquiring, reasoning, and problemsolving (Curriculum Development Council, 2000).

\subsection{Terza fase: valutazione e aggiustamento della bozza di progetto}

Con questo bagaglio linguistico-culturale gli studenti hanno iniziato il lavoro vero e proprio e siamo passati alla fase successiva del progetto. Gli studenti si sono divisi in gruppi di lavoro e hanno pensato a come utilizzare il materiale messo loro a disposizione. Dopo qualche giorno abbiamo ricevuto delle proposte di progetto che abbiamo valutato. In questa fase una di noi è dovuta intervenire perché una delle proposte non sembrava molto appropriata al materiale presentato. Qui si apre un dibattito. Se il progetto è fatto in autonomia, perché l'insegnante è intervenuta a "correggere"/"modificare" l'ispirazione degli studenti? Una risposta è che il progetto doveva riguardare la tematica generale accettata cioè doveva essere uno studio su un quartiere di Firenze come si presentava in passato e come si presenta oggi.

Nel nostro caso non ci sono stati altri motivi per intervenire, ma in generale l'insegnante potrebbe/dovrebbe modificare le proposte degli studenti anche nel caso in cui presentassero un qualcosa che sembra troppo ambizioso. Bisogna infatti essere in grado di valutare la quantità di tempo approssimativamente necessaria per svolgere l'intero progetto. Sarebbe un fallimento se il corso finisse e il progetto rimanesse incompiuto. Inoltre ci potrebbero essere problemi di risorse (persone da intervistare che non sono raggiungibili, documenti difficili da visionare, mancanza di un budget per piccole spese di trasporto, mancanza di mezzi tecnici, ecc.) di cui l'insegnante deve tenere conto quando viene presentata la proposta del progetto (Fried-Booth, 2002).

\subsection{Quarta fase: realizzazione del lavoro}

Fatti dunque i dovuti aggiustamenti alle proposte degli studenti, abbiamo dato il via alla fase numero quattro, quella della vera e propria realizzazione del progetto. Durante la fase dell'elaborazione dei dati i singoli studenti hanno contribuito al lavoro di gruppo a seconda delle loro abilità. Si parla spesso di intelligenze multiple (Gardner, 1983) e in un progetto di questo tipo esse vengono sfruttate al meglio. Infatti, all'interno dei gruppi c'è chi sa scrivere, chi sa disegnare, chi sa usare il computer, chi sa impaginare, chi sa fare fotografie, ecc. Le abilità di ognuno vengono alla luce e tutti si sentono apprezzati e rimangono soddisfatti del proprio contributo. In questo tipo di progetto tutti gli studenti si sen- 
tono parte del prodotto finale, a prescindere dalle proprie capacità linguistiche. Anche lo studente meno capace del corso di lingua può essere di fondamentale importanza per il progetto perché magari è l'unico che sa usare PowerPoint! Un tipo di progetto di questo tipo può essere uno stimolo di enorme importanza proprio per quegli studenti che sono più deboli dal punto di vista linguistico.

Usando le altre abilità (saper disegnare, fare fotografie, ecc.) essi si sentono integrati nella classe più di quanto non lo siano durante una normale lezione di lingua. Non solo, ma attraverso la loro specifica abilità riescono a imparare la lingua meglio che durante la lezione di classe, che evidentemente non viene effettuata secondo il loro naturale modo di apprendimento. In generale si ottiene un miglioramento delle capacità linguistiche in tutti gli studenti, proprio perché ognuno apprende secondo il metodo che più gli è familiare.

Finita la fase dell'elaborazione, gli studenti ci hanno consegnato i propri lavori.

Gli studenti di una classe hanno prodotto un CD-ROM intitolato: "Firenze com'era. Un confronto tra passato e presente". All'interno del CD-ROM hanno inserito i loro lavori che consistono in due prodotti. Il primo è una raccolta di "Pagine di diario" scritte da ogni studente che racconta "Una giornata nella Firenze di Pratolini". Molti dei vocaboli utilizzati sono stati attinti dal testo "Il quartiere" ed è sorprendente come gli studenti siano riusciti a calarsi nelle figure dei protagonisti e a descrivere ambienti e situazioni adeguate al periodo. In ogni "pagina di diario", gli studenti hanno inserito fotografie scattate nel quartiere di Santa Croce. Il secondo prodotto, sempre all'interno del CD, è un lavoro intitolato "Due storie a confronto" ambientate in epoche diverse. La prima storia è un fotoromanzo, "La vita vera", in PowerPoint, ambientato nella Firenze di oggi e la seconda è un racconto, "Francesca e Paolo", ambientato nella Firenze degli anni ' 30 - '40. Il fotoromanzo "narra un giorno della vita di Liz, una ragazza americana in Italia" . Si susseguono fotografie e descrizioni che ritraggono Liz in ogni ora della giornata, a partire dalle sette di mattina alle una di notte nelle diverse attività quotidiane. "Francesca e Paolo", invece, racconta la storia d'amore tra due ragazzi fiorentini negli anni '30 osteggiata dalla loro famiglie. Sia nelle "Pagine di diario" e in "Francesca e Paolo", sia nella "Storia vera" gli studenti hanno messo in evidenza tutti gli aspetti della vita di ieri e quella di oggi, utilizzando il materiale messo a disposizione e le informazioni da loro raccolte nelle diverse attività per la realizzazione del progetto. Anche il linguaggio tiene conto di quanto appreso: così che là dove si descrivono i mezzi di trasporto, si usano vocaboli come "bicicletta", "fiacchere", qua si parla di "motorino", "smart car"; là dove si parla di "veggio" o di "stufa a legna" qua si parla di "riscaldamento" o condizionatori", ecc. Mentre nelle "pagine di diario" e nel racconto il 
linguaggio si è adeguato allo stile de "Il Quartiere", nel fotoromanzo gli studenti hanno usato il linguaggio fresco, giovanile con cui sono venuti a contatto in occasione anche degli incontri organizzati con $i$ giovani. È interessante notare come gli studenti siano riusciti ad assimilare il bagaglio linguistico-culturale trasmesso e a trasferirlo in un testo chiaro, ben strutturato e stilisticamente corretto.

Gli studenti dell'altra classe hanno svolto un lavoro di ricerca sui cambiamenti nel quartiere di Santa Croce dagli anni '30 - '40 a oggi. Hanno intitolato il loro lavoro "Santa Croce: una fetta di Firenze". Il prodotto finale è un libretto stampato su carta di cui gli studenti hanno fatto tutto, perfino la copertina. Il lavoro in sé è diviso in tre parti. Nella prima parte ciascuno studente ha contribuito con una composizione. Si tratta di pagine di diario in cui gli studenti hanno scritto come se stessero vivendo nella Firenze degli anni '30 - '40. Le composizioni sono state ispirate sia dai materiali letti in classe che dagli incontri con i volontari della terza età. Sono stati sicuramente gli aneddoti di queste persone a ispirare le pagine di questi diari, dove si respira veramente un'aria di antichità. La seconda parte raccoglie degli articoli di giornale scritti dagli studenti su alcuni aspetti del quartiere che sono cambiati nel tempo (per esempio l'alimentazione, la descrizione dell'interno delle case, i lavori svolti dagli abitanti e i loro passatempi). Grazie nuovamente agli incontri con i volontari della terza età, ma anche alle escursioni effettuate dagli studenti nel quartiere di Santa Croce, i ragazzi sono stati in grado di scrivere ottimi resoconti dei cambiamenti subiti dal quartiere. Lo stile linguistico è cambiato rispetto alla prima parte. Dal diario, più personale, siamo passati allo stile giornalistico. Gli studenti si sono mostrati versatili e a loro agio in entrambe le produzioni. Le composizioni della prima e della seconda parte sono state arricchite anche da una serie di fotografie che gli studenti avevano fatto durante le loro escursioni nel quartiere. La terza parte è forse quella che ha richiesto maggiore autonomia e dedizione da parte degli studenti. Infatti essi hanno preparato delle mappe diacroniche che mostrano cosa realmente è cambiato in alcune strade del quartiere di Santa Croce dagli anni '30 - '40 a oggi. Per fare questo gli studenti hanno dovuto intervistare i commercianti, gli artigiani e gli abitanti della zona per raccogliere informazioni riguardanti le varie tipologie di negozi e laboratori che c'erano prima di quelli attuali e quindi il lavoro di ricerca è stato abbastanza lungo e ha richiesto un uso piuttosto vario della lingua seconda.

A questo punto ci si è presentato un altro problema, quello della correzione. Il progetto veniva portato avanti in autonomia e quindi non volevamo essere noi a correggere gli elaborati. Inoltre, visto che il progetto è stato elaborato in lingua seconda, non volevamo che con una correzione imposta dalle insegnanti il prodotto finale perdesse di autenticità. Così abbiamo pensato di procedere a una auto-correzione segnalando 
semplicemente gli errori e il loro tipo (tempo verbale non appropriato, concordanza, vocabolario, ecc.). Nella correzione abbiamo privilegiato la sostanza e non la forma, cioè abbiamo corretto solamente laddove il significato non era chiaro. Questo perché abbiamo preferito optare per la correzione di errori "storici" (per esempio abbiamo corretto lo studente che ha scritto nel suo diario fiorentino degli anni '30 che la mattina faceva una bella doccia calda oppure che la sua cena era composta da tre o quattro portate). Il primo tipo di autocorrezione, quella riguardante la grammatica e il vocabolario ha avuto un impatto positivo sugli studenti, in quanto molti di loro si sono resi conto che la maggior parte dei loro errori scritti, sono dovuti per la maggior parte a distrazione (la concordanza, per esempio). Il secondo tipo di errori, e cioè quello relativo alla accuratezza storica, ha mostrato agli studenti che le produzioni scritte non sono fatte solo di pura grammatica e di vocabolario, ma che comprendono anche notizie che devono essere documentate. Gli elaborati quindi sono stati corretti da due punti di vista diversi.

Nella fase di correzione gli studenti si sono trasformati in correttori di bozze e talvolta ci hanno chiesto aiuto per portare delle modifiche ai testi. In questa fase, come del resto in tutte le altre, le insegnanti si sono dimostrate disponibili ad aiutare e incoraggiare gli studenti, ma non hanno imposto la loro presenza. Il ruolo dell'insegnante è stato quello di coordinatore.

\subsection{Quinta fase: presentazione del lavoro e autovalutazione}

Dopo che i vari prodotti erano stati corretti, le insegnanti ne hanno preparata una copia per ciascuno studente (a seconda delle classi, le copie sono state fatte su supporto magnetico oppure su carta). Quando tutte le copie sono state consegnate agli studenti, i diversi gruppi formatisi all'interno di ogni classe, hanno presentato il proprio lavoro. La fase della presentazione è sicuramente una delle più divertenti per tutti, in quanto finalmente gli studenti possono mostrare il prodotto delle loro fatiche ai propri compagni che normalmente sono molto ricettivi e aperti a tutto. La presentazione dà adito a vari scherzi e battute fra i compagni di classe e suscita anche complimenti ai i vari membri dei gruppi (soprattutto per quanto riguarda il tipo di idee che vengono usate e il modo in cui vengono messe in pratica).

Dopo la presentazione in classe dei vari prodotti, le insegnanti hanno promosso una discussione al fine di valutare l'esperienza in sé. In precedenza, durante la fase di presentazione dei materiali, quando gli studenti avevano incontrato i volontari della terza età, essi avevano compilato un questionario di valutazione sull'incontro, rispondendo a domande del tipo: Cosa hai imparato su Firenze e sugli italiani durante l'incontro? Oppure: Pensi che sia stato interessante incontrare queste persone, anche se sono molto più anziane di 
te? Perché? Durante questa fase finale, invece, gli studenti sono stati incoraggiati a riflettere su tutta l'esperienza, dall'inizio alla fine del progetto.

\section{Conclusioni}

La valutazione complessiva del progetto può essere articolata in due componenti: la valutazione degli studenti e la valutazione dei docenti.

Le reazioni degli studenti sono state molteplici, ma anche le più negative sono state accompagnate da una motivazione, che è la cosa più importante per permettere all'insegnante di migliorare in vista di progetti futuri.

Per quanto riguarda le reazioni positive, dobbiamo dire che nella quasi totalità dei casi gli studenti sono rimasti molto soddisfatti del prodotto che hanno creato. Molti di loro si sono detti stupiti, meravigliati e al tempo stesso euforici di avere potuto intervistare parlanti madrelingua e poi scrivere in lingua seconda su argomenti di cui all'inizio del corso non sapevano praticamente niente o quasi. Questo ha indubbiamente aumentato la loro fiducia nelle proprie capacità linguistiche. Altri studenti sono rimasti molto contenti di questo tipo di progetto perché hanno potuto conoscere meglio l'Italia e gli italiani. Le esperienze durante le escursioni nel quartiere di Santa Croce hanno mostrato agli studenti che i fiorentini non sono tutti camerieri e cuochi nei ristoranti del centro, ma che svolgono anche altre occupazioni. Inoltre, allontanandosi dal centro della città che è pieno di turisti e vive sui ritmi dei turisti, gli studenti si sono ritrovati faccia a faccia con una realtà assolutamente diversa, con gente che vive più lentamente e passa volentieri una mezz'ora a parlare con degli stranieri che si interessano al loro lavoro e alla vita della loro città. Attraverso la cultura e il contatto con i fiorentini gli studenti si sono resi conto di aver migliorato la loro lingua, sia per quanto riguarda le loro capacità di ascolto e comprensione, sia per quanto riguarda l'ampliamento del vocabolario.

Per quanto riguarda le reazioni negative, molti studenti si sono lamentati del fatto che il progetto fosse troppo lungo (in realtà un progetto di questo tipo può coprire diverse settimane di corso), mentre altri hanno detto che conciliare lo studio per il corso di lingua e lo studio/ricerca per il progetto li avesse prosciugati di ogni energia. In realtà un progetto come quello da noi svolto richiede una grande quantità di energia e di motivazione. È per questo che ribadiamo l'importanza della prima fase, quella della proposta e presentazione dei materiali. Una soluzione a questo tipo di problema potrebbe essere l'integrazione del progetto nel programma del corso. In parte noi ci abbiamo provato, inserendolo in alcuni test di verifica e negli esami che per il nostro corso sono obbligatori. Evidentemente non abbiamo fatto abbastanza e quindi dobbiamo sviluppare delle idee per migliorare questo aspetto. 
Dal punto di vista dei docenti questa è stata sicuramente una bella esperienza che ha dimostrato a conti fatti come le classi coinvolte avessero acquisito non solo delle conoscenze in più rispetto alle altre, ma anche la possibilità di consolidare l'uso di strutture e vocabolario studiati sia in passato sia durante il semestre corrente. Inoltre, l'uso continuo della lingua seconda in un contesto autentico ha dimostrato quanto beneficio ne abbiano gli studenti per acquisire fiducia nelle loro capacità linguistiche. Sicuramente quest'ultimo aspetto è di enorme importanza per stimolare gli studenti ad approfondire la lingua in studi futuri.

\section{Bibliografia}

Beckett, G. H., "Teacher and student evaluations of project-based instruction". TESL Canada Journal 19/2, 2002, pp. 52-66.

Beckett, G.H. e Slater, T., "The Project Framework: a tool for language, content, and skills integration", ELT Journal Volume 59/2 April 2005, pp. 108-116.

Carter, G., e Thomas H., "Dear Brown Eyes": Experiential learning in a project orientated approach. ELT Journal, Volume 40/3, 1986, pp. 196-204

Ciliberti, A., Manuale di glottodidattica per una cultura dell'insegnamento linguistico, Firenze, La Nuova Italia, 1994.

Collins Cobuild English Dictionary for Advanced Learners, Glasgow, HarperCollins, 2001.

Curriculum Development Council, Learning to Learn. Key Learning Area: English Language Education, Hong Kong, Government Printer, 2000.

Evangelisti, P. e Argondizzo C., "Prefazione" in Evangelisti, P. e Argondizzo, C., a cura di, L'apprendimento autonomo delle lingue straniere - Filosofia e attuazione nell'università italiana, Mannelli (CZ), Rubbettino Editore, Soveria, 2002, pp. 7-14.

Eyring, J.L., Is Project Work Worth It? ERIC document Reproduction Service No. ED 407 838, 1997, consultabile al seguente indirizzo elettronico: $<$ http://www.georgejacobs.net/Annotated_Bibliography___L2_and_ CL.rtf> [01/07]

Ferragatti, M., e Carminati E., "Airport: An Italian Version", Modern English Teacher, 2/4, 1984, pp. 15- 17.

Fried-Booth, D. L., "Project Work with Advanced Classes", ELT Journal, 36/2, January 1982, pp. 98-103.

Fried-Booth, D. L., Project Work, Oxford, Oxford University Press1986-1992-1997-2002 .Gallacher, Lynn, "Project Work with Teenagers", consultabile al seguente indirizzo elettronico: <http://www.teachingenglish.org.uk/think/methodology/project_work.shtml> [12/08]

Gardner, H., Frames of the Mind: The Theory of Multiple Intelligences, New York, Basic Books, 1983.

Haines, S., Projects for the EFL Classroom: Resource Material for Teachers, Walton-on-Thames, Surrey, U K, Nelson, 1989. 
Landolfi, L., "Autonomia e didattica delle lingue" " in Evangelisti, P. e Argondizzo, C. a cura di L'apprendimento autonomo delle lingue straniere - Filosofia e attuazione nell'università italiana, Mannelli (CZ), Rubbettino Editore, Soveria, 2002.

Legutke, M., "Project Airport: Part 1”, Modern English Teacher, 11/4, 1984 pp. 10-14.

Legutke, M., "Project Airport: Part 2" Modern English Teacher, 12/1, 1985, pp. 28-31.

Legutke, M. e Thomas, Process and Experience in the Language Classroom, London: Longman, 1991.

Little, D. "Learner Autonomy and Second/Foreign Language Learning." Guide to Good Practice for Learning and Teaching in Languages, Linguistics, and Area Studies. The Higher Education Academy: Subject Center for Languages, Linguistics, and Area Studies. University of Southampton, 2003. Southampton, UK.

Moss, D., e Van Duzer, C., "Project-Based Learning for Adult English Language Learners", consultabile al seguente indirizzo elettronico: <http:// www.ericdigest.org/1999_<4/project.htm> [12/08]

Papandreou, A., "An Application of the Projects Approach to EFL", English Teaching Forum, 32/3, 1994, pp.41-42.

Sheppard, K., e Stoller, F., "Guidelines for the Integration of Student Projects in ESP Classrooms", English Teaching Forum, 33/2, 1995, pp. 10-15.

Stoller, F.L. "Project work: A Means to Promote Language and Content", Forum 35/4, October - December 1997, p. 2 consultabile al seguente indirizzo elettronico: <http://exchanges.state.gov/forum/vols/vol35/no4/ p2.htm> [01/07]

Ward, G. 1988. "I've Got a Project on", New South Wales, Australia: Primary English Teaching Association, consultabile al seguente indirizzo elettronico:

< http://exchanges.state.gov/forum/vols/vol35/no4/p2.htm> [01/07]

\section{Pratolini e Firenze}

Pratolini, V., Il Quartiere, Milano, Mondadori, 1961.

Giannelli, L., a cura di, Sull'Arno d'argento - ricordi fiorentini attraverso mezzo secolo, Firenze, Scramasax, 2003.

Cesati, F., La grande guida delle strade di Firenze - Storia, aneddoti, arte, segreti e curiosità della città più affascinante del mondo attraverso 2400 vie, piazze e canti, Roma, Newton \& Compton Editori, 2003.

<http://www.comune.firenze.it/firenze900/scroce.htm> [01/07] (dove è possibile trovare informazioni sul quartiere di Santa Croce e i suoi esercizi storici)

<http://www.comune.firenze.it/firenze900/visite2.htm> [01/07] (dove è possibile prenotare visite guidate nel quartiere di Pratolini)

$<$ http://www.viapinzochere6.it> [12/08] (dove è possibile trovare informazioni sulle varie strade del quartiere di Santa Croce) 


\title{
AUTONOMIA DI APPRENDIMENTO LINGUISTICO IN CONTESTO MIGRATORIO: IL CASO DEI LAVORATORI \\ DI ORIGINE STRANIERA
}

\author{
Raymond Siebtcheu \\ Università per Stranieri di Siena
}

\section{Introduzione}

Con l'allargamento dell'Unione Europea e la libera circolazione delle persone all'interno degli Stati consentiti, la necessità di imparare altre lingue è diventato un obbiettivo cardine da raggiungere. Così « Il CEFR (Common European Framework of Reference for Languages: Quadro Comune Europeo di Riferimento per la conoscenza delle Lingue), altrimenti citato con l'acronimo QCER (Quadro Comune di Riferimento Europeo), rappresenta una linea guida impiegata per descrivere i risultati conseguiti da chi studia le lingue straniere in Europa, nonché allo scopo di indicare il livello di riferimento di un insegnamento linguistico negli ambiti più disparati »'. Dall'inizio degli anni Novanta in avanti, si assiste dunque ad un forte interessamento dell'Europa riguardo alle questioni linguistiche in vista dell'integrazione dei cittadini europei e non europei. L'apprendimento delle lingue (compreso anche quello autonomo) diventa così uno strumento notevole per l'acquisizione della cittadinanza partecipativa europea.

L'auto-apprendimento è, in effetti, uno dei principali temi che nasce come supporto al processo di apprendimento di una lingua. Questi ultimi anni, il tema in esame ha assunto un valore molto forte non solo perché la lingua è (ed è sempre stata) una delle chiavi principali per l'integrazione in un paese, ma anche perché i progressi della tecnologia stanno ridefinendo nuove forme di apprendimento in particolar modo, quelle svolte in contesto di autonomia.

Le migrazioni interne e esterne insieme ai progressi tecnologici (video-conferenze) sono alcuni aspetti che hanno portato la 'lingua dell'altro' ad essere al centro delle attenzioni. Studiare una lingua oggi è una necessità assoluta. Imparare velocemente, da solo, secondo i propri criteri e stili di apprendimento e secondo i propri obiettivi sono sicuramente le ragioni che hanno portato i potenziali apprendenti a tuffarsi nel sistema dell'auto-apprendimento sfruttando così le nuove offerte tecnologiche.

Di fronte a un tema così importante, i contributi scientifici sono naturalmente amplissimi, ma la complessità dell'argomento si materializza da 
una rassegna di espressioni, in larga misura in lingua inglese, che «spesso sottintendono concetti analoghi, strettamente collegati o addirittura identici: self-learning, self-study, self-teaching, self-education, self-instruction, selfplanned learning, self-directed learning, self-access learning, autonomous learning, individualised instruction, $o$, in francese, auto-apprentissage, apprentissage autodirigé e autonomie guidée» (Serra Borneto, 2003). Ma non è questa la sede per affrontare le diverse accezioni inerenti all'auto-apprendimento. Per familiarità e comodità, ma anche per semplificare il quadro di riferimento, per affrontare le questioni legate al tema in esame daremo all'espressione autoapprendimento «un'accezione neutra e generica, riferendoci con esso a quei contesti e a quelle situazioni in cui l'apprendente si trova, o per sua scelta o per necessità, a studiare senza il continuo e diretto controllo dell'insegnante, il quale può comunque esercitare, nei tempi e nei modi stabiliti, una fondamentale funzione di guida e supervisione» (Serra Borneto, 2003).

\section{Autonomia di apprendimento e auto-apprendimento}

Fra le condizioni che permettono a un apprendente di sviluppare un apprendimento autonomo, cioè «essere cosciente dei propri obiettivi e consapevole dei metodi e degli strumenti che più si adattano alle proprie preferenze e alle proprie inclinazioni, significativa è la possibilità di scegliere fra percorsi, materiali e attività» (Brookes A., Grundy P., 1988). Con questa illustrazione, ci rendiamo conto che l'autonomia di apprendimento non è altro che la capacità di intraprendere un percorso di apprendimento che tenga conto delle proprie esigenze e della propria disponibilità. Si tratta dunque di un apprendimento personale (senza il continuo e diretto controllo dell'insegnante) e personalizzato (secondo le proprie esigenze). Tra i fattori che possono garantire il successo dell'apprendimento autonomo si può citare: la motivazione da una parte e la quantità di input accessibile e disponibile dall'altra parte. In questo senso, chi apprende autonomamente deve essere «fortemente motivato verso il suo oggetto di studio, pienamente autonomo e dotato delle capacità necessarie a intraprendere un tale cammino, capace cioè di distinguere chiaramente i suoi obiettivi e di trovare il modo per raggiungerli» (Serra Borneto, 2003). Quindi la libertà di scegliere fra una vasta gamma di materiali deve essere funzionale a portare l'apprendente a migliorare le proprie competenze linguistiche. L'autonomia di apprendimento che, come abbiamo visto, implica libertà ma anche responsabilità di apprendimento, dovrebbe pertanto portare ad un autonomia linguistico-espressiva dopo aver superato lo stato di sopravivenza linguistica.

L'auto-apprendimento dal canto suo, può essere considerato come un modello didattico che fa riferimento ad un processo in cui grande peso hanno le nuove tecnologie (che al contrario possono anche essere assenti 
in percorsi di apprendimento autonomo) in modo che possa offrire «input linguistico, stimoli per l'esercitazione, correzione automatica, grammatica di riferimento, glossario, e così via)" (Balboni P., 1996). Naturalmente l'auto-apprendimento non implica soltanto l'utilizzo di un computer e i suoi accessori, ma anche le attrezzature audio-video; e la presenza di una biblioteca e di una sala lettura.

\section{Il binomio lingua e immigrazione}

Chi si occupa di linguistica e immigrazione sa bene che dagli anni Ottanta del ventesimo secolo, il quadro teorico e didattico dell'insegnamento delle lingue straniere in Europa è cambiato con l'ingresso massiccio e sempre crescente delle popolazioni immigrate. Proprio per questo motivo, « La descrizione e l'analisi della diversificazione linguistica apportata dalle migrazioni è proprio l'oggetto di uno dei pochi Centri di eccellenza della ricerca, costituiti dal ministero dell'Università: uno, appunto, è stato creato presso l'Università per stranieri di Siena e ha per compito la costituzione di un Osservatorio linguistico permanente dell'italiano all'estero e delle lingue immigrate in Italia» (Vedovelli, 2002). L'arrivo degli immigrati in Italia non si è dunque limitato a cambiare il panorama socioculturale del paese, ma ha suggerito una nuova riformulazione dei percorsi di apprendimento linguistico. Chi guarda le lingue immigrate da lontano pensa a un clima di tensione e di guerra linguistica con la lingua ospite ${ }^{2}$. Chi le guarda da vicino si rende, invece, conto che la loro presenza rende l'insegnamento e lo studio dell'italiano meno monotono e più dinamico. In realtà, insegnare l'italiano ad apprendenti parlanti lingue lontane tra di loro e tipologicamente diverse dall'italiano è sicuramente una ricchezza per tutti. Il contatto dell'italiano con altre lingue, che, in parte, è una delle conseguenze di questa nuova situazione, non si limita soltanto all'interno della classe o della scuola ma si verifica anche in tutti gli ambiti della società. E per Vedovelli «'L'italiano come lingua di contatto' significa considerare la competenza linguistico-comunicativa come luogo in cui codici linguistici e culturali diversi si incontrano e producono nuove identità» (Vedovelli, 2002).

Fatta questa premessa, ci rendiamo conto che il tema dell'autonomia di apprendimento è piuttosto complesso; sopratutto se si riferisce ad una particolare categoria di apprendenti come i lavoratori di origine straniera.

\section{Il lavoratore di origine straniera}

In Italia quando si parla di lavoratore immigrato si tende a pensare a persone povere e non alfabetizzate che svolgono lavori manuali e usuranti. 
Bisogna invece dire che molti degli immigrati presenti in Italia, impegnati nei lavori umili, arrivano in Italia in possesso di un titolo di studio (maturità, diploma, laurea). In effetti, secondo una ricerca condotta dal gruppo Cerfe $^{3}$, oltre la metà $(53 \%)$ degli stranieri presenti in Italia è in possesso di una laurea conseguita nel proprio paese di origine o in Italia, ma rischia una "dequalificazione" a causa del mancato riconoscimento del titolo di studio e dalle lunghe procedure burocratiche necessarie per consentire un'integrazione professionale che rispecchia il titolo di studio in possesso. Il lavoratore di origine straniera molto spesso è dunque costretto a svolgere attività lavorative che non sono attinenti alla sua competenza professionale. Spinti dal coraggio, dall'umiltà, ma anche dalla reale necessità economica, i lavoratori migranti sono pronti a svolgere qualsiasi mansione. Ma è utile ricordare che il cittadino migrante svolge queste mansioni non perché non ha la preparazione necessaria per avere incarichi importanti, ma perché la società e il sistema per l'impiego gli offre solo questi tipi di lavoro.

Non dimentichiamo, però, che molti immigrati stranieri, laureati nei loro paesi o in Italia, sono riusciti ad integrarsi ai livelli apicali nella società italiana (istituzioni pubbliche e private: Università, Banche, Testate radio-televisive e Stampa, Organizzazioni Non Governative...).

Per tutte queste categorie di lavoratori di origine straniera, ci concentreremo sulla fascia più debole cioè quella che ha più difficoltà ad integrarsi a causa di alcuni aspetti sociali: l'analfabetizzazione ${ }^{4}$ (Tebaldi G., 1990) e le mansioni lavorative socialmente basse.

\section{La questione della lingua}

L' aspetto linguistico è sicuramente uno dei punti cruciali riguardo all'integrazione degli immigrati lavoratori. Mentre i lavoratori che hanno fatto il loro percorso scolastico e/o universitario in Italia non hanno problemi linguistici, o se ce l'hanno sono molto minori, i lavoratori stranieri che arrivano in seguito ai decreti flussi, ai ricongiungimenti famigliari o per altri motivi sono maggiormente confrontati al problema linguistico. Anche se le loro motivazioni sono diverse dai classici studenti stranieri europe ${ }^{5}$ o dagli studenti non comunitari presenti in Italia da diversi anni, i lavoratori di origine straniera sanno che hanno fatto un lungo viaggio per trovare un lavoro e quindi sono pronti a fare qualsiasi cosa per integrarsi nei migliori dei modi. Così la lingua diventa una delle barriere da sormontare. Il dizionario tascabile, possibile unico strumento di apprendimento del lavoratore straniero, non ha un'importanza secondaria rispetto agli attrezzi del centro linguistico universitario che è messo alla disposizione degli studenti universitari. L'autonomia di apprendimento diventa così, un percorso tanto delicato quanto relativo. Se gli studenti stranieri, gli uomini d'affari stranieri o i funzionari internazionali im- 
parano la lingua italiana per un obiettivo specifico e sicuramente gratificante, è utile ricordare che molto spesso il percorso di apprendimento interviene dopo un protocollo di accoglienza e di integrazione da parte delle istituzioni che ricevono questi apprendenti. I lavoratori immigrati hanno invece un atteggiamento diverso pure sapendo di dover superare la barriera linguistica per riuscire ad integrarsi nel giusto modo. In realtà, questi nuovi cittadini non riescono ad impegnarsi a fondo in un percorso di apprendimento autonomo efficiente quando le loro condizioni di integrazione sociale (almeno quelle primarie) non sono garantite. La non frequentazione, o in alcuni casi, la non assiduità ai corsi d'italiano viene quindi giustificata dai legittimi bisogni sociali non garantiti già dall'inizio dei corsi programmati. Vedovelli afferma, in questo senso, che «L'accoglienza, infatti, rappresenta un ambito dominato dalle scelte della società ospite, e la regolarizzazione è una questione che i clandestini vivono in termini di assenza. L'abitazione, la salute e l'assistenza, il lavoro (nel senso generale dell'attività in cui il migrante è occupato), la socializzazione e il tempo libero sono componenti intrinseche di ogni processo migratorio. Lo è meno, invece, la formazione: il bisogno di inserirsi in percorsi di sviluppo delle competenze professionali, alfabetiche, linguistiche, generalmente culturali appare strutturarsi formalmente solo in una piccola parte dell'emigrazione straniera in Italia» (Vedovelli, 2002). Diventa quindi difficile parlare di un continuo ed efficiente autonomia di apprendimento da parte del lavoratore di origine straniera senza parlare d'integrazione sociale. In realtà, solo un'autonomia socioculturale nel paese di arrivo può consentire al lavoratore straniero di intraprendere un percorso autonomo di apprendimento linguistico funzionale. La lingua, secondo noi, è la chiave della città, che viene consegnata al lavoratore migrante al momento del suo ingresso nel paese. E così l'immigrato che non riceve questa chiave non entrerà mai, socialmente parlando, in questa città essendo costretto a rimanere davanti alla porta (della città) e a guardare da lontano, o forse neanche, le delizie della civiltà della città 'sbarrata'. Se la lingua assume per l'immigrato lavoratore un valore estrinseco, è semplicemente dovuto all'assenza di una politica programmatica d'integrazione che dovrebbe essere attivata al momento dell'ingresso e non a metà percorso come spesso succede.

Questa riflessione sulla questione della lingua per l'immigrato lavoratore ci porta ad analizzare da vicino le ragioni che possono costituire un freno nel percorso di apprendimento autonomo del migrante. In realtà, come abbiamo già detto, il lavoratore immigrato sviluppa comunque un'autonomia di apprendimento, anche se i risultati non sono tali da garantire un'autonomia linguistica per interagire in tutti gli ambiti della società. Il nostro compito è invece quello di vedere che cosa impedisce il lavoratore a migliorare il suo percorso di autonomia. Il livello soglia ${ }^{6}$, che il Consiglio d'Europa, negli anni Novanta, considera come il livello 
dell'«autonomia comunicativa» (Vedovelli, 2002:65) deve, forse, essere per il lavoratore di origine straniera il livello di partenza per garantire un'efficiente continuità nel suo cammino verso l'autonomia? Un'analisi sul comportamento linguistico del lavoratore immigrato ci aiuterà forse a trovare qualche elemento di risposta a questa domanda.

\section{Il comportamento linguistico del lavoratore di origine straniero}

\subsection{Il ruolo della L2}

Anche se l'Italia non è più un paese di transizione, ma piuttosto una meta di immigrazione stabile, la questione della lingua da parte dei lavoratori stranieri non è risolta. Possiamo, infatti, affermare che la stragrande maggioranza dei lavoratori immigrati ha una motivazione di tipo professionale (cioè finalizzata alla ricerca o alla conservazione del proprio lavoro) anche perché secondo la vigente normativa sull'immigrazione in Italia, l'immigrato che perde il suo lavoro ha sei mesi per poter trovare un altro lavoro, altrimenti viene espulso. Si tratta quindi di una motivazione che non privilegia lo studio della lingua ospite di fronte ai bisogni primari quali la famiglia, la casa, e il lavoro. Pure essendo importante e di estrema utilità, l'apprendimento della L2 viene spesso considerata dagli stessi immigrati come una perdita di tempo nel senso che il tempo impiegato per studiare la lingua poteva essere usato per lavorare e guadagnare soldi. Quindi la lingua non è percepita come una fonte di ricchezza economica. Ma ricordiamo che questa situazione si verifica non solo in Italia, ma in tutti i paesi d'immigrazione che trascurano la fase di accoglienza e di integrazione sociale dei nuovi cittadini.

\subsection{L'apprendimento spontaneo}

La linguistica acquisizionale dell' italiano L2 ha avuto una forte spinta propulsiva a partire dalla diffusione di un tipo di apprendente nuovo per la nostra lingua, ovvero gli immigrati stranieri, i quali hanno come tratto caratteristico il fatto di non sviluppare la propria competenza in italiano L2 primariamente entro un processo formativo, in un contesto scolastico, ma innanzitutto nello scambio comunicativo spontaneo con i parlanti nativi con i quali interagiscono. L'ingresso di un immigrato adulto in una classe di lingua sin dal primo momento del percorso migratorio, appena arrivato in Italia, è un caso non così frequente come quello dell'inserimento in classe quando ormai il migrante ha sviluppato un qualche livello di competenza spontanea in italiano L2 (Vedovelli, 2002).

Per motivi di priorità, come abbiamo spiegato prima, ma anche per motivi economici, la maggiore parte degli immigrati sviluppa la propria 
competenza attraverso l'apprendimento detto 'spontaneo' cioè legato al contatto con le persone di madrelingua o altri stranieri che parlano correntemente l'italiano. Si tratta però di un apprendimento che sviluppa un «input non controllato che dipende dai rapporti sociali nei quali l'immigrato è inserito" (Vedovelli, Massara, Ramat, 2001). Pur avendo una quantità di input particolarmente elevata, ci preme sottolineare che la qualità dell'output prodotto non garantisce purtroppo una competenza linguistica autonoma. L'apprendimento spontaneo è quindi un tipo di apprendimento autonomo che si sviluppa, però, con un alto grado d'incontrollabilità. Per questo, Dickinson sottolinea l'importanza di un lavoro di gruppo quando ricorda che "Molti allievi autonomi lavorano con altri nel loro apprendimento perché l'autonomia non implica l'isolamento» (Dickinson, 1987).

\subsection{Il livello di competenza}

Anche se la lingua non è una priorità per le ragioni legittime che abbiamo ricordato prima, il lavoratore immigrato impara comunque la lingua italiana. Notiamo però che il fatto che la sua motivazione sia diminuita a causa dei suoi bisogni primari lo porta a limitarsi ad un un 'italiano di contatto' (costruzione linguistica scritta o orale da parte dello straniero, che nasce dopo il contatto quotidiano e continuo con i parlanti di madre lingua o altri parlanti stranieri che padroneggiano l'italiano; ma nasce anche con l'utilizzo dei mezzi di comunicazione di massa: televisione, radio, internet). Naturalmente qui ricordiamo che stiamo parlando di lavoratori integrati nelle categorie occupazionali basse. Anche perché sarebbe difficile assumere un lavoratore straniero in alcuni livelli apicali se non ha una buona conoscenza linguistica. La L2 viene dunque trascurata non perché non è importante, ma semplicemente perché il livello raggiunto dal lavoratore (vedi figura 1) gli basta per compiere le sue mansioni quotidiane.

\subsection{L'uso e la diffusione delle lingue immigrate}

Le lingue immigrate in Italia, già eredità linguistica dei migranti, costituiscono per gli italofoni e per l'Italia una grande ricchezza. E così molti enti locali e associazioni organizzano dei corsi per imparare le lingue immigrate. Abbiamo ad esempio dei corsi di lingua e cultura araba, cinese, albanese... per cittadini italiani e bambini stranieri nati in Italia. Ma è importante che ci siano dei laboratori interculturali e interlinguistici in modo che «i bambini di origine straniera trovino gli strumenti per definire la propria identità, ma dove anche i bambini di origine italiana abbiano la possibilità di entrare in contatto con un patrimonio linguistico diverso da quello tradizionalmente proprio dello spazio idiomatico d'origine» (Vedovelli, 2002). 
Ma bisogna ricordare che l'uso delle lingue immigrate è a volte esclusivo e l'italiano viene trascurato o dimenticato. Nelle famiglie immigrate ci sono dei genitori che usano esclusivamente le loro lingue d'origini. In alcune comunità immigrate c'è una situazione di chiusura nei confronti dell'esterno. "A questo proposito è esemplare il caso delle comunità cinesi in Italia, che, concentrandosi in uno spazio circoscritto, tendono a costituire nuclei sociali separati. Numerosità e radicamento nel tempo e nello spazio sono una condizione di possibilità per la visibilità, ma nelle comunità cinesi c'è la tendenza alla non apertura nei confronti della comunità ospitante» (Bagna, Barni, Siebetcheu, 2004). In questo senso, Vedovelli aggiunge dicendo che «la stabilizzazione crea anche le condizioni per far uscire dall'isolamento il singolo migrante e per fargli ricostruire una trama di rapporti con gli altri connazionali: da qui la nascita di centri di aggregazione della comunità immigrata, lo sviluppo di manifestazioni comunitarie formali e informali, la creazione delle condizioni per l'uso sistematico della lingua originaria» (Vedovelli, 2002).

\subsection{I bambini-docenti}

A differenza dei genitori che per ragioni dovute alla motivazione, all'età e all'uso delle loro lingue d'origine, mettono la lingua italiana al secondo posto, «la formazione è tratto intrinseco del percorso migratorio, invece, per i bambini e gli adolescenti, cioè per i figli della prima generazione di migranti, arrivati con i genitori o ad essi ricongiuntisi successivamente all'arrivo di questi in Italia o nati nel nostro paese: bambini e adolescenti, infatti, sono soggetti all'obbligo scolastico; i loro genitori no» (Vedovelli, 2002). I genitori chiedono infatti le consulenze dei loro figli per risolvere alcune questioni che necessitano una certa competenza linguistica: la gestione di alcuni testi scritti e orali. I bambini immigrati, sapendo leggere e scrivere sviluppano una certa efficacia comunicativa e la gestione di determinati ambiti della comunicazione che i genitori con l'apprendimento spontaneo non riescono a fornire.

\subsection{Il rapporto tra uso linguistico e compito professionale}

Anche se gli immigrati lavoratori preferiscono avvicinarsi alla lingua attraverso un contesto di apprendimento non formale, ricordiamo che il posto di lavoro è un potenziale luogo di apprendimento. E quindi potrebbe essere sfruttato per accompagnare il lavoratore nel suo percorso di apprendimento. E così «molti enti di formazione professionale impartiscono al loro interno l'insegnamento della lingua italiana agli stranieri, direttamente collegato ad uno specifico corso tecnico o come attività continuativa di supporto» (Scaglione, 2004). Per lo svolgimento delle loro mansioni gli immigrati si limitano a parole e espressioni legate alla loro attività lavora- 
tiva. La povertà linguistica dei migranti non è dovuta soltanto alla mancata integrazione, ma anche alla limitatezza linguistica dei lavori che essi svolgono. In realtà, per molte delle mansioni riservate agli immigrati serve ben poco la lingua italiana. Per Minuz, nella programmatica didattica, c'è da mettere in evidenza un possibile rischio "che un'educazione linguistica modellata su ambienti di lavoro comunicativamente ridotti offra possibilità linguistiche "povere". Il luogo di lavoro non richiede che una minima padronanza della lingua: alcuni lavori in ristoranti, imprese di pulizia, nei campi che non offrono particolari opportunità comunicative. Ciò che gli immigrati apprendono in questi casi non consente loro di esprimere stati d'animo e opinioni, di accedere autonomamente a servizi e informazioni, insomma di muoversi in autonomia nella società ospite senza la mediazione di connazionali o altre figure e può condurre a forme "cristallizzate" di lingua impoverita» (Scaglione, 2004).

Questi punti, che non sono esaustivi, illustrano in parte le ragioni che condizionano il cammino verso l'autonomia di apprendimento dei lavoratori di origine straniera.

\section{La questione del materiale didattico}

Nella didattica dell'italiano agli immigrati è importante concentrare l'attenzione non solo sull'aspetto didattico-formativo, ma anche sull'aspetto socioculturale che costituisce la vera barriera al problema di tutti i tipi di apprendimento, compreso quello autonomo. Bisogna quindi attivare delle strategie che consentono all'apprendente di impegnarsi per la propria formazione linguistica. In questo senso, Dickinson sostiene che «Il termine "autogestione" descrive un atteggiamento verso l'apprendimento in cui l'allievo accetta responsabilità riguardo al medesimo ma non esclude affatto la possibilità, per lo studente, di seguire un corso convenzionale». (Dickinson, 1987). Servono dunque strumenti (materiale: audio, video, cartaceo; e struttura polivalente: biblioteca, laboratorio linguistico e ufficio di orientamento e di collocamento...) facilmente accessibili all'immigrato; anche tenendo conto del suo luogo di abitazione e della sua disponibilità rispetto ai suoi orari di lavoro. La gratuità dell'accesso a questi servizi garantirebbe una cospicua e regolare frequentazione. Come già detto, la didattica dell'italiano per immigrati stranieri dovrebbe pensare anche agli aspetti sociali della vita dell'apprendente. Un centro di informazione e di auto-apprendimento potrebbe ad esempio consentire all'immigrato di avere accesso a internet per poter comunicare con i parenti o seguire delle lezioni di informatica. Anche perché oggi per usare efficientemente gli strumenti dei laboratori linguistici è importante aver seguito un percorso di alfabetizzazione informatica. Naturalmente in un centro di questo tipo, mirato all'auto-apprendimento dei 
lavoratori immigrati, visto che l'ambiente dei laboratori linguistici delle università potrebbe essere meno adatto per loro, è importante che ci sia una figura in grado di capire i problemi sia linguistici sia socioculturali degli apprendenti ma soprattutto in grado di motivarli a frequentare regolarmente il Centro. L'ideale sarebbe che questa figura sia quella di un immigrato formato e competente che sia in grado di incoraggiare l'apprendente a studiare, e a frequentare il Centro. In questo modo, anche nei casi in cui l'apprendente non avrà voglia di studiare avrà almeno voglia di andare a salutare il suo concittadino presente al Centro. Si tratta forse di una mossa decisamente rilevante per garantire la motivazione.

La lingua si può spostare ovunque l'immigrato si trova (casa, comunità immigrate, lavoro ecc.). Come esempio, possiamo citare il progetto di apprendimento della lingua e cultura italiana pubblicato su Metropoli di Repubblica e curato dall'Università per Stranieri di Siena nel 2006. Si tratta di un progetto che entra proprio nella logica della motivazione di cui si parlava. Gli esperti e docenti implicati nel progetto hanno, in effetti, trasformato l'inserto in un materiale didattico adeguato all'apprendimento autonomo. L'inserto settimanale Metropoli, molto letto per le notizie di politica migratoria ma anche per le preziose informazioni riguardanti i paesi di provenienza degli immigrati, è stato in quel periodo ancora più letto proprio grazie all'offerta formativa che si è infiltrata nella vita sociale dell'immigrato. Questo progetto (Vignette 1,2,3) ci fa vedere che l'uso della stampa come materiale didattico può anche essere un mezzo di apprendimento autonomo.

\section{Dall'autonomia di apprendimento all'autonomia linguistica}

Come abbiamo cercato di dimostrare, è molto difficile per un lavoratore immigrato sviluppare un percorso di autonomia di apprendimento che "garantisce un buon livello di autonomia linguistica» (Vedovelli, 2002) senza essere passato da un apprendimento guidato. La formula d'apprendimento ideale per lui sarebbe dunque: apprendimento guidato + apprendimento spontaneo fino almeno al livello $\mathrm{B}^{7}$ che garantisce il possesso di una competenza linguistico-comunicativa adeguata a gestire in modo autonomo le interazioni sociali nei contesti della quotidianità. L'autonomia di apprendimento non si deve limitare soltanto all'abilità orale ma deve anche concentrarsi sull'abilità della scrittura (che l'apprendimento spontaneo riesce difficilmente a garantire). "Nel caso delle lingue straniere è ben noto che esistono allievi che apprendono, almeno all'inizio, attraverso l'analisi e lo studio delle strutture grammaticali della lingua, i quali non si sentono in grado di affrontare attività più nettamente comunicative se prima non hanno raggiunto una sufficiente sicurezza su questo terreno» (Serra Borneto, 2003). Queste dichiarazioni 
confermano quanto detto prima sull'apprendimento guidato e sul livello di partenza rispetto all'apprendimento autonomo.

All'inizio di questo articolo abbiamo detto che l'autonomia non deve essere soltanto la capacità di imparare da solo, ma anche quella di andare il più lontano possibile con la competenza comunicativa. L'autonomia di apprendimento deve quindi portare ad una autonomia linguistica. «In questo senso, ogni apprendente, guadagnando sempre maggiore fiducia in se stesso e nelle sue possibilità, dovrebbe arrivare a essere in grado di valutare autonomamente il livello raggiunto e le relative capacità e conoscenze» (Sheerin, 1989). Qui però è importante soffermarsi sulla scelta del materiale didattico. All'inizio del percorso, non è prudente lasciare l'apprendente scegliere il materiale non solo perché non sa dove iniziare ma soprattutto perché non sa ancora dove arrivare. Zorzi è d'accordo con questo punto di vista quando suggerisce che «'interazione fra studenti e istituzione deve avvenire anche sul piano dell'educazione alla partecipazione. È necessario accettare che non tutti gli studenti né sono abituati a un lavoro autonomo, né lo desiderano, e quindi il bisogno di gestire da soli il proprio apprendimento deve, in qualche modo, essere "indotto". Ciò significa che l'istituzione deve prevedere materiali e attività che si configurino come "formazione dell'apprendente verso l'autonomia", in cui cioè il percorso per arrivare a imparare qualcosa comprenda sia procedure e contenuti indicati dall'insegnante, sia momenti gestiti integralmente dallo studente» (Zorzi, 1995).

Figura 1

A

Livello elementare

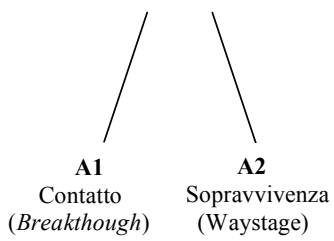

B

Livello intermedio

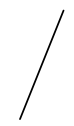

B1

Soglia

(Threshold)

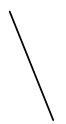

B2

Progresso (Vantage)
C

Livello avanzato
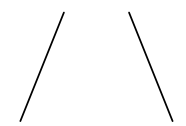

C1

Efficacia

(Effective

Operational Proficiency)

Fonte: Consiglio d'Europa, 2002:30

Questo schema ad albero riassume quanto detto sull'autonomia di apprendimento da parte dei lavoratori di origine straniera. In base ai livelli stabiliti dal Quadro comune europeo per le lingue, l'apprendimento autonomo può portare fino al livello di padronanza linguistica. L'autonomia di apprendimento che il lavoratore costruisce già dal primo giorno di contatto con la lingua e con gli italofoni si può quindi sviluppare fino ad un livello di autonomia linguistica. 
Vignette 'Metropoli'

Vignetta 1

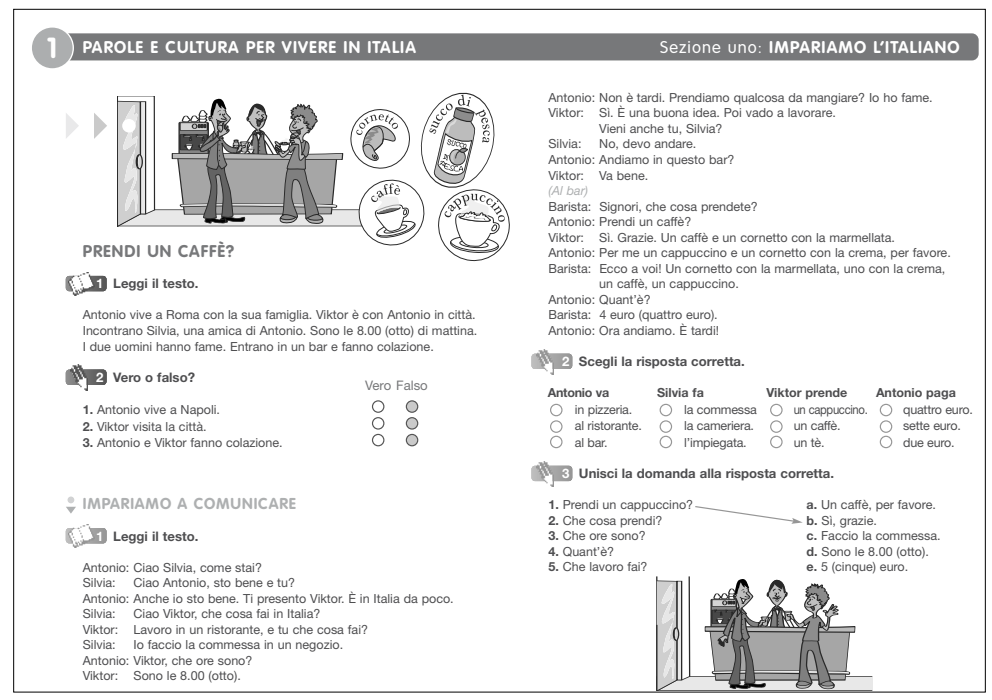

Uno degli obbiettivi di questa vignetta è di sviluppare la capacità di lettura e di comprensione da parte del lettore-apprendente. Un altro obbiettivo è quello di migliorare la capacità comunicativa fornendo una vasta gamma di elementi lessicali e culturali che arricchiranno sicuramente i discorsi dei lettori-apprendenti.

Vignetta 2

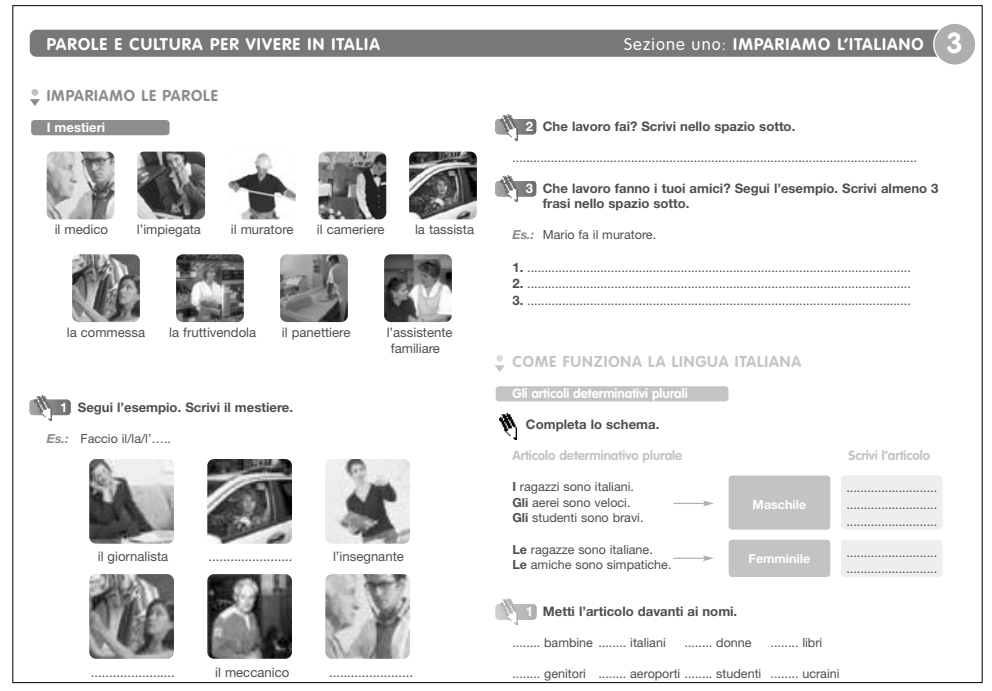


Questa vignetta aiuta il lavoratore a identificare la propria professione ma a familiarizzarsi anche con le altre professioni. Le nozioni grammaticali illustrate danno la possibilità di migliorare la competenza linguistica dell'apprendente. Viene sviluppata anche l'abilità di scrittura.

Vignetta 3

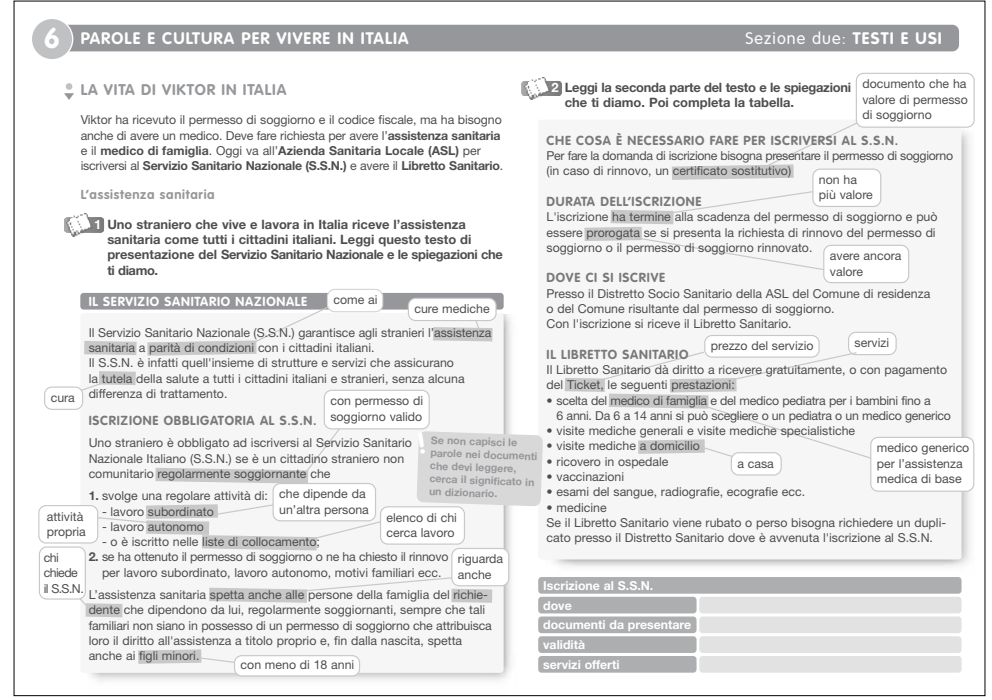

La vignetta 3 illustra brevemente come funziona il Sistema Sanitario Nazionale usando un linguaggio settoriale chiaro, semplice e successivamente spiegato in didascalia. L'obbiettivo non è solo di superare le barriere create dal linguaggio burocratico spesso incomprensibile, ma anche di familiarizzare l'utente straniero al nuovo sistema sanitario.

\section{Conclusioni}

In conclusione, possiamo ribadire che l'autonomia dell'apprendimento da parte dell'immigrato è forse molto più incontrollabile rispetto ad altri apprendenti, ma abbiamo visto che il lavoratore straniero ci può arrivare se considera il supporto iniziale dell'insegnante e successivamente del tutor (dopo aver raggiunto il livello soglia). "Il concetto di 'autonomia', che nell'insegnamento scolastico viene perseguito come meta educativa generale, diviene dall'Università in poi un concetto operativo, organizzativo. Nei centri linguistici si devono fornire guida e strumenti a persone già autonome nei processi cognitivi, negli stili d'apprendimento, nella gerarchia degli scopi del loro sforzo di apprendere, nella focalizzazione degli interessi e delle motivazioni - ma anche nella gestione del tempo 
per lo studio, nella necessità di autovalutazione continua nell'organizzazione pratica del lavoro. E la mutltimedialità inserita in una prospettiva "umanistico-affettiva" può rispondere alle esigenze di autonomia" (Balboni, 1995).

In sostanza, in questo lavoro, abbiamo tentato di fare capire che l'autonomia di apprendimento è possibile anche senza attrezzatura tecnologica e quindi dal primo contatto con la lingua, ma il lavoratore di origine straniera deve portare la sua autonomia di apprendimento ad un livello di autonomia linguistica che gli consente di risolvere alcuni compiti complessi. L'apprendente deve quindi impegnarsi per un continuo ampliamento degli usi linguistici tale da dominare una vasta gamma di situazioni comunicative. Solo così il lavoratore straniero potrà interagire non solo in tutte le situazioni informali e formali di comunicazione, ma anche in quelle professionali.

\section{Bibliografia}

Bagna C., Machetti S., Vedovelli M., 2003, Italiana e lingue immigrate: verso un plurilinguismo consapevole o verso varietà di contatto? In: A. Valentini, P. Mulinelli, P.cuzzolin, G. Bernin (a cura di), Ecologia linguistica, Atti del XXXVI Congresso Internazionale di Studi della società di Linguistica Italiana, Bergamo 26-28 settembre 2002, Bulzoni, Roma: 201-222.

Bagna C., Barni M., Siebetcheu R., 2004, Toscane Favelle. Le lingue immigrate nella provincia di Siena, Guerra Edizioni, Perugia.

Balboni P.E.,1994, Didattica dell'italiano a stranieri, Bonacci, Roma.

Balboni P.E., 1996, Elementi di glottodidattica, La Scuola, Brescia.

Balboni P.E., 1991, Tecniche didattiche e processi d'apprendimento linguistico, Liviana, Padova.

Balboni P.E., 1998, Tecniche didattiche per l'educazione linguistica. Italiano, lingue straniere, lingue classiche, Utet Libreria, Torino.

Banfi E., 1986, L'italiano degli altri, in "Italiano \& Oltre", I, n.5 pp.231-234.

Bargellini C. (a cura di), 2000, Nǒ Hăo e Salam. Lingue e culture a scuola, ISMU, Milano.

Barni M., Villarini A. (a cura di), 2001, La questione della lingua per gli immigrati stranieri. Insegnare, valutare e certificare l'italiano L2, FrancoAngeli, Milano.

Benucci A., 1998, Linsegnamento della lingua e cultura italiana in situazioni di lingue e culture a contatto. In: E. Tannini, Atti del Corso di formazione iniziale per il personale della Scuola Secondaria da destinare all'estero (30 settembre - 30 ottobre 1986), Ministero degli Affari Esteri - Università per Stranieri di Siena, Siena.

Celentin P., 2000, Autonomia e supporti informatici e telematici nell'insegnamento dell'italiano a stranieri. In: Dolci R. - Celentin P., pp. 201-217

Ciliberti A., 1994, Manuale di glottodidattica. Per una cultura dell'insegnamento linguistico, La Nuova Italia, Firenze. 
Ciliberti A. (a cura di), 1981, L’insegnamento linguistico per scopi speciali, Zanichelli, Bologna.

Borneto Serra C. (a cura di), 2003, C'era una volta il metodo. Tendenze attuali nella didattica delle lingue straniere, Carocci, Roma.

Brookes A., Grundy P. (a cura di), 1988, Individualization and autonomy in language learning, ELT Documents 131.

Centro di Documentazione Educazione degli adulti del Lazio, 2004, Italiano per migranti. Apprendimento della lingua 2 e inserimento nel mondo del lavoro, FrancoAngeli, Milano.

Coveri L., 1991, Problemi e bisogni linguistici e sociolinguistici degli studenti europei in Italia : un sondaggio tra studenti del progetto Erasmus, Franco Angeli, Milano. In: Mazzoleni M., Pavesi M., pp. 129-144.

Comodi A., 1995, Materiali autentici: selezione e uso nella didattica dell'italiano come lingua straniera, Guerra Edizioni, Perugia.

Consiglio d'Europa, 2001, Common European Framework of Reference dof Languages: Learning, teaching, assessment, Cambridge University Press, Cambridge.

Consiglio d'Europa, 2002, Quadro comune europeo di riferimento per le lingue: apprendimento, insegnamento, valutazione, La Nuova Italia, Oxford

Danesi M., 1988, Manuale di tecniche per la didattica delle lingue moderne, Armando, Roma.

Demetrio D. (a cura di), 1984, Immigrazione straniera e interventi formativi, Franco Angeli, Milano.

Demetrio D., 1992, Bisogni comunicativi e risposte della comunicazione della formazione nell'immigrazione extracomunitaria, La Nuova Italia, Firenze. In Tassinari G., pp.193-216.

De Marco A. (a cura di), 2001, Manuale di glottodittatica, Carocci, Roma.De Mauro T., 1994, Capire le parole, Carocci, Roma.

De Mauro T., 2002, Prima lezione sul linguaggio, Carocci, Roma.

Diadori P. (a cura di), 2001, Insegnare italiano a stranieri, Le Monnier, Firenze.

Dickinson L., 1987, Self-instruction in Language Learning, Cambridge University Press, Cambridge.

Favaro G. (a cura di), 1999, Imparare l'Italiano. Imparare in Italiano. Alunni stranieri e apprendimento della seconda lingua, Guerrini e Associati, Milano.

Favaro G., 1987, Italiano lingua seconda. Proposte per una formazione linguistica degli immigrati stranieri in Italia. Metodi e unità didattiche, Franco Angeli, Milano.

Felici A., 1994, Il rapporto italiano-dialetto nell'apprendimento spontaneo dell'italiano come lingua seconda da parte di adulti immigrati. In Vedovelli n.2 SILTA XXIII

Fratter I., 2004, Tecnologie per l'insegnamento delle lingue, Carocci, Roma.

Freddi G., 1979, Didattica delle lingue moderne, Minerva Italica, Bergamo.

Freddi G., 1970, Metodologia didattica delle lingue straniere, Minerva Italica, Bergamo.

Freddi G. (a cura di), 1987, L'insegnamento della lingua-cultura italiana all'estero, Aspetti glottodidattici, Le Monnier, Firenze.

Freddi G., 1999, Psicologia, sociolinguistica, glottodidattica, Utet Libreria, Torino.

Galli De' Paratesi N., 1981, Livello soglia per l'italiano, Consiglio d'Europa, Strasburgo. 
Gensini S., Vedovelli M., 1990, Teoria e pratica del Glotto-kit, Franco Angeli, Milano.

Giacalone Ramat A., 1988, L'italiano e le altre lingue: strategie di acquisizione, Il Mulino, Bologna.

Giacalone Ramat A., Italiano di stranieri. In Sobrero A.A., 1993, PP.341-410

Giacalone Ramat A., Vedovelli M.( a cura di), 1994, Italiano lingua seconda / lingua straniera, SLI, Bulzoni, Roma.

Grassi R., Valentini A., Bozzone C. (a cura di), 2002, L'italiano per lo studio nella scuola plurilingue tra semplificazione e facilitazione. Atti del ConvegnoSeminario Alunni stranieri nella scuola: l'italiano per lo studio, Bergamo, 1719 giugno 2002, Perugia, Guerra.

Grezzi C., Guerini F., Mulinelli P. ( a cura di), 2003, Italiano e lingue immigrate a confronto: riflessioni per la pratica didattica. Atti del Convegno-Seminario Bergamo, 23-25 giugno 2003, Guerra, Perugia.

Iori B. (a cura di), 2005, L'italiano e le altre lingue. Apprendimento della seconda lingua e bilinguismo dei bambini e dei ragazzi immigrati, FrancoAngeli, Milano.

Lepschy A.L., Tamponi A.R. (a cura di), 2005, Prospettiva sull'italiano come lingua straniera, Guerra Edizioni, Perugia.

Maccarelli A., 2001, L'italiano e le lingue altre nella scuola multiculturale, Edizioni ETS, Pisa.

Maestro G., 2004, Italiano: lingua straniera. Educazione linguistica e integrazione culturale nelle scuole superiori, Guerra-Edizioni, Perugia.

Mariani L.(a cura di), 1994, L'autonomia nell'apprendimento linguistico, La Nuova Italia, Firenze.

Nuccetelli G. (a cura di), 2000, Scrivi come mangi. La didattica dell'italiano L2 nei Centri territoriali permanenti per l'istruzione e la formazione in età adulta, FrancoAngeli, Milano.

Pallassini A., 2006, Le strategie comunicative degli immigrati del quartiere Esquilino di Roma: i manifesti di carattere politico, Pacini Editore, Roma. "SILTA" Studi Italiani di Linguistica Teorica e Applicata, XXXV, 3: 605-626.

Porcelli G., 1992, Le tecnologie glottodidattiche in un approccio umanistico-affettivo. In "SeLM", XXIII, 3: 3-8.

Porcelli G., 1988, Computer e glottodidattica, Liviana, Padova.

Ramat A.G., 2003, Verso l'italiano. Percorsi e strategie di acquisizione, Carocci, Roma.

Scaglione S. (a cura di), 2001, L'identità linguistica e culturale all'estero, Bulzoni Editore, Roma.

Scaglione S. (a cura di), 2001, L'identità linguistica e culturale degli stranieri in Italia: insegnamento e acquisizione dell'italiano, Bulzoni Editore, Roma.

Semplici S. (a cura di), 1995, Proposte per l'auto-apprendimento. Atti del III Seminario Permanente dei Centri Linguistici Siena - Firenze 20 - 22 febbraio 1995, Università per Stranieri di Siena, Siena.

Sheerin S., 1989, Self-access, Oxford University Press, Oxford.

Stanchina C., 1975, The Logic of Autonomy as a Strategy for Adult Learners, CRAPEL.

Tebaldi Giovanni, 1990, Lo sviluppo dei popoli, La Scuola, Brescia. 
Trentin G, (a cura di), 1996, La didattica in rete, Garamond, Roma.

Trentin G. ( a cura di), 1992, Telematica e didattica, Menabò, Ortona.

Vaccarelli A., 2001, L'italiano e le lingue altre nella scuola multiculturale, Edizioni ETS, Pisa.

Vedovelli M., 1992, L'italiano e le altre lingue: aspetti linguistici e culturali. Modulo 2, Documento MILIA. Materiali per gli Insegnanti di Lingua Italiana Aggiornamento. dovelli M. (a cura di), 2000, La lingua degli immigrati stranieri in Italia, in Studi di Emigrazione, n. 140 (numero monografico).

Vedovelli M., 1981, La lingua degli immigrati stranieri in Italia, in LEND, n.3, pp.17-23.

Vedovelli M., 1991, L'immigrazione straniera in Italia: note tra sociolinguistica e educazione linguistica, in "SILTA", XX, pp. 411-435.

Vedovelli M.,1990, Apprendimento e insegnamento dell'italiano in contesto migratorio: un bilancio sui modelli e sugli interventi per l'immigrazione recente, in "Percorsi” VIII, PP.233-250.

Vedovelli M., 1994, Apprendimento e insegnamento linguistico in contesto migratorio: dall'apprendimento spontaneo a quello guidato dell'italiano L2, Pacini Editore, Roma. "SILTA", XXIII, 2: 193-220.

Vedovelli M., 1994, L'italiano parlato dagli italiani e l'italiano appreso dai non italiani. In: De Mauro T. (a cura di), Come parlano gli italiani, La Nuova Italia, Firenze, pp. 87-98.

Vedovelli M., 1999, Indagini sociolinguistiche nella scuola e nella società italiana in evoluzione, FrancoAngeli, Milano.

Vedovelli M., Massara S., Giacalone Ramat A. (a cura di), 2004, Lingue e culture in contatto, FrancoAngeli, Milano.

Vedovelli M., 2002, Guida all'italiano per stranieri, Carocci, Roma.

Vedovelli M., Barni M., Bagna C., Troncarelli D., Pizziconi S., Totaro I., Pallassini A., Semplici S., 2006, Corso di Lingua Italiana, "Metropoli" di Repubblica, 11 lezioni, settembre-dicembre 2006.

Vergaro C., 1998, Nuove tecnologie e didattica delle lingue. In: Serra Borneto C.,pp. 305-324.

Zangola M.T., 2000, Techniche didattiche e glottotecnologie. In: De Marco A., pp.141-153.

Zorzi D., Leone P., Prospettive ed applicazioni per l'insegnamento dell'italiano L2, Besa Editrice, Lecce.

Note

${ }^{1}$ www.wikipedia.org

${ }^{2}$ Per un approfondimento sulla descrizione delle lingue immigrate viste come simboli di "pace linguistica" e di dialogo con la lingua dominante, rimandiamo a Bagna C., Barni M., Siebetcheu R. (2004).

${ }^{3}$ Articolo di Elisabetta Norzi sul sito http://www.edscuola.com/archivio/handicap/immigrazioneinchiesta.htm - Giovedì 22 novembre 2007, alle ore 18:46:26 Educazione\&Scuola $\mathbb{C}$ 
${ }^{4}$ Non disporre delle basi fondamentali per l'istruzione, cioè non sapere né leggere né scrivere. Oggi si potrebbe anche parlare di analfabetismo informatica, per chi non sa usare gli strumenti informatici; o di analfabetismo culturale, quando si verifica qualche carenza nel padroneggiare una competenza, in ambiti specifici, che interessa un maggior numero di persone.

${ }^{5} \mathrm{Ci}$ riferiamo particolarmente agli studenti impegnati nei programmi di mobilità ( Erasmus, Socrates, Comenius... all'interno delle Università dell'Unione Europea).

${ }^{6}$ Il livello soglia è il grado di competenza che permette al parlante di acquisire il primo, importante livello di autonomia linguistica. Corrisponde al profilo B della scala dei livelli stabiliti dal Consiglio d'Europa. Un apprendente con livello soglia vanta secondo Vedovelli (2002:65) un' "autonomia comunicativa".

${ }^{7}$ Fa parte della scala a 6 livelli (A1, A2, B1, B2, C1, C2) predisposta dal Quadro comune europeo per le lingue.

${ }^{8}$ Le vignette 1,2 e 3 sono tratte dalla seconda lezione del corso d'italiano pubblicata su "Metropoli" nr.29 del 17 settembre 2006. La lezione è curata dall'Università per stranieri di Siena (Bagna C., Barni M., Pallassini A., Pizziconi S., Semplici S., Totaro I., Troncarelli D., Vedovelli M.). Il corso d'italiano, curato interamente da l'Università per stranieri, è composto di undici lezioni; e ogni lezione è divisa in due sezioni. " La prima (che è intitolata "Impariamo l'italiano") fornisce gli elementi di base della lingua, la seconda (intitolata "Testi e usi") ricrea le situazioni più frequenti nella vita quotidiana (le istituzioni, il lavoro, gli ospedali, le scuole) fornendo quei testi che gli uffici forniscono agli immigrati per spiegargli che cosa fare ma che spesso contengono parole della burocrazia incomprensibili anche per gli italiani (per esempio, salutare, presentarsi, conoscere altre persone, l'arrivo in aeroporto, i primi contatti con l'ufficio immigrazione della questura, i moduli per il permesso di soggiorno, il codice fiscale e così via)" (http://metropoli.repubblica.it/corsoitaliano/index.html). 


\title{
L'AUTONOMIA DI APPRENDIMENTO DELLE LINGUE NEL BIENNIO UNIVERSITARIO: LA DIDATTICA ALL'INSEGNA DEL COSTRUTTIVISMO CON CLASSI VIRTUALI E RICERCHE ONLINE
}

\author{
Christiane Büchel \\ Università degli Studi di Firenze
}

\section{Introduzione}

Riflessioni sull'uso dei nuovi media nella didattica, in special modo di internet, si collocano all'interno del dibattito sull'autonomia di apprendimento, che è contestualizzato nella discussione su nuovi approcci e nuove mentalità dell'apprendere collegata alla richiesta europea del "life long learning"1.

La richiesta dell" "imparare ad imparare" (learning to learn) - "il modo di imparare socialmente più utile in assoluto"- implica concetti di autonomia nell'agire dei discenti ${ }^{2}$, mentre i paradigmi tradizionali dell'insegnamento non riescono a soddisfare pienamente le nuove necessità. Per poter imparare per tutta la vita bisogna prima di tutto sapere come avvicinarsi autonomamente allo studio, come scegliere argomenti/ materie, come definire lo scopo dello studio e come scegliere gli strumenti giusti per giungere a questo scopo.

Gli strumenti della Commissione Europea per lo studio delle lingue, il portfolio delle lingue, e specialmente lo strumento della language biography, che mirano ambedue ad una maggiore motivazione dei discenti nell' apprendimento delle lingue e alla capacità individuale di procedere coscientemente ed autonomamente nello studio, sono espressioni coerenti di questa nuova esigenza di autonomia ${ }^{3}$.

La recente discussione didattica a sua volta si orienta al pensiero del costruttivismo, che ha le sue radici nelle scoperte della psicologia cognitiva e la neurolinguistica e ha innescato un ripensamento generale: Si parla di un cambio dei paradigmi nell'interpretazione dei processi di comunicazione e di apprendimento, concretamente del passaggio dal paradigma trasmissivo al paradigma interattivo. Nella didattica vorrebbe dire che il sapere non viene più trasmesso dall'insegnante per essere immagazzinato o elaborato ma che il sapere viene costruito dai discenti stessi ${ }^{4}$. Si propone agli insegnanti di costruire il sapere interagendo con i discenti, i quali partecipano con un'autonomia finora mai raggiunta nell'ambito dell'educazione scolastica-universitaria. L'apprendimento è 
allora visto come un impegno attivo da parte dei discenti a costruire la propria conoscenza, piuttosto che come travaso della conoscenza dalla mente del docente a quella dello studente. In questa maniera il sapere viene generato dinamicamente nella mente del discente come qualcosa di personale. Al centro dell'attenzione sta il discente, i suoi bisogni e le sue risorse, specie il sapere preesistente, in quanto nuovi contenuti vengono sempre costruiti con riferimento alle conoscenze già esistenti (learner-centered-learning).

Un filone di questo nuovo approccio è strettamente legato all'uso di internet in aula. Il costruttivismo sembra essere entrato nell'orizzonte della didattica (delle lingue) anche, e prima di tutto, per il fatto della sempre maggiore diffusione di internet che ha cambiato il modo di acquisire conoscenze e resa possibile una maggiore possibilità di autonomia. Internet ha generalizzato l'accessibilità di certe fonti del sapere in maniera enciclopedica ed è diventato un mezzo di informazione, di comunicazione e trasmissione di strumenti

Mentre "l'autoapprendimento" e "l'apprendimento autonomo" (e con questo anche il tradizionale e-learning) appartengono ancora al paradigma trasmissivo, "l'autonomia di apprendimento" del discente nella pianificazione e nelle scelte del suo percorso formativo all'insegna del costruttivismo è un concetto che attiene ad un paradigma nuovo e didatticamente ancora nella fase di sperimentazione: Di lì deriva un approccio didattico che rende la figura dell' insegnante più che mai centrale, necessaria e indispensabile, anche se in una funzione fondamentalmente diversa da prima.

Il mio contributo pone come obiettivo di riassumere alcuni pensieri legati al termine di "autonomia di apprendimento" e di presentare dei progetti didattici con mezzi informatici realizzati all'interno del lettorato di tedesco della Facoltà di Lettere e Filosofia dell'Università di Firenze.

Nel marzo 2007 si è svolto a Firenze il convegno "New Millennium Learners - Scuole, tecnologie, apprendimento" organizzato dall'OECD e dall'Agenzia Nazionale per lo Sviluppo dell'Autonomia Scolastica, dove venne posto tra l'altro la domanda sullo stato dell'arte relativa alle tecnologie digitali nell'apprendimento. Punto di partenza era la convinzione che i profondi cambiamenti nel modo di comunicare avvenuti recentemente implichino la necessità di "ridisegnare" nuove modalità di insegnamento, capaci di migliorare le attività didattiche e valorizzare le abilità di ogni singolo studente, sfruttando le conoscenze preesistenti nell'individuo e le sue specificità: Riflessioni su come sviluppare abilità di studio utili non solo per un corretto approccio alla lingua straniera, ma applicabili ai loro studi in generale.

Di fronte ai New Millennium Learners, la generazione di giovani nati a partire dagli anni ' 80 che non hanno mai conosciuto un mondo privo di internet e dei media digitali, si percepì l'urgenza di realizzare scenari 
educativi innovativi, presupponendo che siano cambiati (o da cambiare?) le capacità cognitive dei giovani, i loro modi di comunicare e di conseguenza anche i modi apprendere. Questo anche per ridurre il rischio di un digital disconnect tra il mondo della formazione e il mondo delle pratiche sociali dei ragazzi legati all'utilizzo massiccio dei nuovi mezzi di comunicazione. Questa nuova generazione sta proprio ora entrando nel mondo delle università, il quale dovrebbe seriamente interrogarsi come agire di fronte ai cambiamenti descritti.

\section{I nuovi compiti dell'insegnante: educazione, costruzione, istruzione}

Una mente abituata alla figura dell'insegnante come istanza di tipo tradizionale richiederà automaticamente di essere guidata nella scelta della materia, nel percorso di studio e nella scelta delle mete che meritano di essere raggiunte. Un sistema scolastico o universitario dove prevale l'idea che l'insegnante debba guidare il pensiero dello studente fino al punto che questo possa ricapitolare nozioni e nessi propri della persona dell'insegnante o dell'autore di un libro, non rende certo facile la realizzazione dei concetti di autonomia e del life long learning che implicano la disponibilità mentale e la capacità metodologica dei discenti per acquisire prevalentemente in autonomia nuove competenze e campi di conoscenza.

Sarà il compito futuro degli insegnanti stessi di creare soggetti autonomi che siano in grado di continuare autonomamente il loro processo educativo per tutta la vita. Starà agli insegnanti rendere i discenti, che finora sono stati percepiti come oggetto dell'intervento dell' insegnante, soggetti del proprio apprendimento. Gli insegnanti giocheranno un ruolo fondamentale nella creazione di autonomia di apprendimento nel discente e acquisiscono in questa maniera un ruolo fondamentale e indispensabile - a scapito dei timori diffusi che l'autoapprendimento o l' autonomia di apprendimento potessero rendere superflua la figura dell'insegnante. Più di contenuti e convenzioni gli insegnerà come intraprendere vie e percorsi del pensiero senza voler automaticamente predefinire dove si debba arrivare. L'insegnante dovrà essere orientato verso la costruzione autonoma del sapere e cercare di pilotare il discente il meno possibile.

Una volta cosciente, dove vorrà arrivare (scelte indotte anche dalla società , dal mondo di lavoro, da altri fattori esterni), lo studente cercherà i mezzi e contenuti che lo portano alla meta: Secondo i principi costruttivistici il sapere è di per sé inconcluso e si costruisce individualmente e in relazioni sociali. L'apprendimento in tale senso diventerà un processo attivo con riferimenti pluridimensionali.

Il compito principale del insegnante sarà allora di insegnare le competenze per l'acquisizione e l'uso del sapere (motivazione per l'apprendimento, giudizio critico, saper imparare). Dovrà essere in grado di proporre 
ad ogni discente la via appropriata per un apprendimento individuale e significativo, rinunciando ad un singolo metodo di insegnamento. Ci sono tanti metodi quanti discenti, i quali decidono sulla tematica, il tempo da dedicare e gli approcci metodologici.

Iniziative didattiche potrebbero essere insegnamenti aperti, progetti didattici, iter investigativi di apprendimento, durante i quali l'insegnante interviene prima di tutto nel momento iniziale, garantendo poi la continuità dell'impegno. Al contrario della tradizione didattica il discente in questo contesto non si deve confrontare con risposte ma con domande.

La figura e il ruolo dell'insegnante si dirige automaticamente verso nuovi orizzonti, descritti nella letteratura con termini del tipo mentore, trainer, coach, facilitator - derivanti direttamente dal mondo del management aziendale. Anche gli studenti dovranno applicare i loro metodi di studio a criteri di efficienza, e farsi un proprio "time-management" finora piuttosto estraneo al loro modo di studiare.

Bisogna allora chiedersi dove si possa in aula concretamente delimitare l'istruzione per dare spazio alla costruzione autonoma del sapere. Come sarà possibile, limitando i tentativi di trasmettere sapere, di garantire un progresso nelle competenze linguistiche? Fino a che punto e in quale momento l'insegnante può stare in sottofondo senza perdere la sua funzione di garante di un progresso nell'apprendimento significativo? È nel tentativo di mettere in atto nuove strategie didattiche consoni alle nuove esigenze e i nuovi paradigmi, che offro da diversi anni corsi di ricerche web in aula, con la navigazione autonoma e presentazioni multimediali.

\section{Educare all'autonomia per l'apprendimento significativo}

Nel contesto descritto l'educazione all'autonomia nell'apprendimento diventa una necessità di prima categoria. Già all'inizio del percorso di apprendimento gli studenti vanno guidati a diventare soggetti del proprio progresso. Devono essere in grado di esprimere delle autovalutazioni, di programmare metodicamente il loro iter di apprendimento e tracciare in un diario il loro percorso di apprendimento, per poter verificare e dimostrare in ogni momento lo stato del loro progresso.

L'autonomia di apprendimento è per forza limitato finché mancano gli strumenti metodici dello studiare, ma anche, nel caso preciso dell'insegnamento delle lingue, le conoscenze di base in una lingua straniera. Quando si tratta, invece, di approfondire le conoscenze base (a partire dal livello B1), subentra con dinamica crescente l'aspetto dell'autonomia di apprendimento, dove l'insegnante ha il compito di limitare progressivamente le sue induzioni per dare spazio alla iniziativa ed agli interessi degli stessi discenti - seguendo principi fondati sulle linee guida della 
supposizione costruttivista. Bisognerà promuovere la maturità individuale, l'autocoscienza, l'autodisciplina e la responsabilizzazione del discente.

Partendo dal presupposto che si fa sempre più necessaria l'autonomia di apprendimento, anche l'uso dei nuovi media trascenderà i limiti dell' autoapprendimento pilotato (e-learning classico) per aprirsi alle svariate possibilità di percorsi individualizzati. Internet è una fonte di linguaggio autentico - anche se settoriale - accessibile da chiunque, dovunque ed in qualsiasi momento. Prima di internet era molto difficile per uno studente aderire di iniziativa propria a fonti autentiche senza un supporto da parte dell'insegnante o da parte di istituzioni di mediazione culturale. Il mondo virtuale con la vasta gamma di possibilità di contatti autentici con le altre lingue e culture sta creando un profondo cambiamento nel pensare lo studio delle lingue.

Particolarmente adatti per la realizzazione dei nuovi concetti sono ricerche web ed webquests, attività che sono caratterizzate da tante scelte individuali non prestabilite dall'insegnante. La premessa è la creazione di ambienti virtuali all'interno delle quali possano agire i discenti e l'insegnante.

Vorrei delineare in seguito i tentativi intrapresi concretamente negli ultimi anni per mettere in atto i nuovi principi di autonomia e costruzione nel contesto dell'insegnamento della lingua tedesca con supporto informatico.

\section{La creazione di ambienti/classi/aule virtuali}

Per attivare un corso con mezzi informatici ci sono alcune premesse indispensabili che riguardano sia aspetti prettamente tecnici e legali, sia aspetti psicologici e didattici. Un corso che prevede l'uso di internet ha bisogno di essere prima di tutto istaurato online, cioè deve essere identificato con un preciso sito web. Le alternative prese in considerazione in questo contesto sono i weblog e le piattaforme didattiche (Moodle).

L'ambiente virtuale del corso deve garantire, pur essendo in rete, una definizione della cerchia d'utenza che ha il diritto d'accesso, delle fonti pubblicate su questo sito e degli strumenti per comunicazioni interrelazionali. Questo sia per creare un ambiente riservato in cui muoversi disinvoltamente, sia per regioni prettamente legali: Solo creando una specie di ambiente protetto è garantito il copyright per qualsiasi elaborato pubblicato nell'ambiente virtuale, e, viceversa, l'uso di fonti esterne non comporta il rischio di offendere il copyright altrui.

Nella "classe" virtuale è possibile comunicare in maniera multidirezionale e asincronico tra l'insegnante ed i discenti e tra discenti: Essi ricevono materiale da parte dell'insegnante, scambiano pareri sia con l' insegnante sia tra di loro e consegnano il materiale elaborato in manie- 
ra visibile a tutti. L'insegnante da il suo parere sui lavori degli studenti online. Questo approccio elimina in gran parte l'uso di materiale cartaceo: Documenti proposti dall'insegnante sono visibili sul sito, e nella stessa maniera gli elaborati degli studenti. Discorsi individuali vengono resi pubblici al momento che i discenti mettono a conoscenza i propri risultati non solo all'insegnante, ma a tutto il gruppo. Questa forma di lavorare in pubblico (alquanto ristretto perché limitato al numero degli iscritti al corso) risulta spesso un esperienza nuova non sempre facile per i discenti. Mentre nella consegna di un elaborato i rapporti esclusivi e riservati con gli insegnanti sono collaudati fin dai tempi della scuola, una presentazione a tutti i partecipanti viene vissuto non di rado come imbarazzante.

Nell'aula virtuale vengono stabilite alcuni criteri base, per esempio la durata delle unità tematiche, con data di inizio e fine (che spesso coincide con la data di consegna di elaborati di riferimento) dell'unità e l'ordine di grandezza delle comunicazioni (stabilito tecnicamente con filtri e maschere). L'aula virtuale impone una maggiore disciplina che tende ad astrarre dalla persona dell'insegnante: Spesso le premesse stabilite dal sistema vengono rispettate in maniera molto diligente dai discenti.

Nell' approccio qui proposto, le comunicazioni online si sommano a quelle date nei periodi trascorsi settimanalmente insieme in aula. L'aula virtuale è allora uno sdoppiamento di un gruppo che comunque si costituisce in contemporanea materialmente in un contesto convenzionale di lezione in aula. O meglio: Le iscrizioni nell'ambiente virtuale sono il primo atto da fare durante la prima lezione in aula. L'ambiente virtuale può essere considerato in questo contesto un portale sia per ricerche autonome sia per comunicazioni fuori dell'orario della lezione. In questa maniera, una classe resta un gruppo stabilito anche oltre la lezione in aula. Viene creato in tal modo un nesso continuo tra i partecipanti che può in qualsiasi momento della settimana essere attivato e utilizzato per scambi di informazione. Questa condizione crea l'impressione di continuità utile per tenere sempre alto, durante tutta la settimana, il livello di attenzione alle tematiche e alle vicende del corso, rendendo più responsabili i discenti di fronte ai propri compiti da affrontare. Anche per l'insegnante, questo va detto, questa presenza continua virtuale è molto impegnativa e trascende di gran lunga i compiti e orari convenzionalmente e contrattualmente definiti.

\subsection{La creazione dell'aula virtuale usando Weblogs}

Per creare una classe virtuale l'insegnante istituisce un weblog con il titolo del corso e le prime comunicazioni. Durante la prima lezione, i partecipanti al corso, che si svolge durante tutto il semestre con ritmo settimanale in un aula informatica, si fanno registrare nello stesso pro- 
gramma creatore di blog, ricevendo in questa maniera una identità virtuale nel sistema.

L'insegnante del blog del corso invita ora tutti gli iscritti a entrare nella schiera dei suoi "amici". Fatto questo, l'accesso al blog viene riservato a tutti gli "amici" chiamati e non è più visibile nella sfera pubblica del www. Dopo la registrazione i discenti creano a loro volta la propria pagina, un proprio blog, aprendolo nella maniera descritta a tutti partecipanti del corso: Ognuno invita tutti gli altri a far parte del suo gruppo amici.

Ogni settimana sul blog principale del corso, creato dall'insegnante, appaiono i compiti da svolgere dagli studenti. Il lavoro proposto consiste di regola nella definizione di un problema o dalla descrizione di uno scenario, seguite da alcune domande o compiti da svolgere nel contesto descritto. Indispensabile è che il compito proposto sia formulato in modo chiaro e che sia ben delineato in tutti i suoi dettagli. Le informazioni per svolgere il compito vanno trovate navigando nel www: L'insegnante propone una serie di link di partenza adeguati. I risultati dei studenti vengono alla fine del semestre presentati con l'uso di PowerPoint (una ventina di diapositive circa), sul weblog individuale o con una pagina html.

\subsection{La creazione dell'aula virtuale usando una piattaforma didattica}

Le esperienze fatte nella gestione dei corsi in base a weblogs sono poi ultimamente confluite in una nuova sperimentazione didattica, cioè la creazione di una classe virtuale con il sistema open source Moodle.

La piattaforma didattica open source Moodle, introdotta negli ultimi anni anche nelle realtà universitarie italiane, apre delle possibilità di gestione di classi e gruppi del tutto nuove che nell'essenza tendono a rivoluzionare la distribuzione dei ruoli sia dei discenti sia degli insegnanti universitari. Il sistema stesso di questo programma è stato pensato in altri contesti culturali e impone, una volta introdotta nella realtà didattica universitaria d'Italia, nuovi paradigmi del tutto estranei al sistema tradizionale dell'insegnamento. Richiede sia dagli insegnanti, sia dai discenti un radicale ripensamento dei ruoli che svolgono in aula: Il discente diventa automaticamente più responsabilizzato del suo agire, mentre l'insegnante si distanzia progressivamente dal ruolo di somministratore di sapere.

L'iscrizione dei partecipanti al corso con una parola chiave (durante la prima lezione in aula) permette all'insegnante di controllare il numero e le modalità delle iscrizioni: I discenti sono tenuti a formulare immediatamente nella lingua straniera un profilo della propria persona che può essere in un secondo momento (eventualmente da casa) modificato ed arricchito di una foto propria.

Appena effettuata l'iscrizione, l'insegnante ha gli strumenti predisposti da Moodle per controllare ogni attività svolta dai discenti durante la settimana. Anche i partecipanti tra di loro possono seguire le attività 
individuali di tutti i partecipanti attraverso gli elenchi di attività registrate e pubblicate su Moodle nel corso di una settimana. La funzione del calendario rende possibile la programmazione dettagliata di ogni settimana del semestre: I discenti possono rendersi conto fin dall'inizio del semestre dell' iter tematico delle lezioni e della complessità dei compiti da svolgere da settimana in settimana. I forum, istaurati dall'insegnante per ogni argomento, rendono possibili discussioni online legate direttamente a delle sfere tematiche. In più la funzione del wiki invita i discenti a creare dei documenti collettivi online, sempre legati agli argomenti settimanali. I compiti proposti dall'insegnante possono consistere sia in un catalogo di domande da risolvere con ricerche nel web, sia in una serie di link obbligatori ma non esclusivi. Inoltre l'insegnante può fornire su Moodle (in upload) materiali testuali o multimediali fondamentali per gli argomenti in discussione.

Indispensabile resta l'incontro settimanale in aula, sia per fornire conoscenze tecniche per un utilizzo corretto della piattaforma da parte dei partecipanti, sia per discutere i risultati tematici elaborati dai discenti e messi a conoscenza di tutti nei vari contenitori a disposizione (forum, wi$k i$, chat tracciati durante la settimana).

\section{Esperienze didattiche dell'insegnamento della lingua tedesca con base informatica}

\subsection{Premesse}

\subsubsection{I contenuti didattici}

La ricerca Web è sempre legata ad un interesse di tipo contenutistico. Nel nostro caso si è trattato dell'insegnamento della lingua attraverso la riflessione sulla storia del mondo di lingua tedesca, in particolar modo della storia tedesca del secondo dopoguerra. Si potrebbe scegliere anche altri argomenti che focalizzano la cultura, le attualità, la mentalità, la costituzione e la società tedesca. Di fatto, però, nelle navigazioni in internet, i campi tematici si sovrappongono automaticamente e uno studio di accento storico comporta sempre anche di doversi confrontare con gli altri campi elencati qui sopra.

\subsubsection{Premesse metodiche per la navigazione autocontrollata: Il diario di lavoro}

I discenti mettono tutte le loro annotazioni e poi anche i loro risultati/elaborati sul loro personale blog accessibile da tutti i partecipanti del corso e dall'insegnante, e tutti possono immediatamente commentarlo. Durante la loro navigazione annotano continuamente una traccia del loro procedere nel proprio documento online. Molto utile per l'autocon- 
trollo del discente è un diario di lavoro, in cui prende nota di ogni passo percorso e di ogni pagina web utile che frequenta.

In funzione del diario crea in una finestra un documento di testo che rimane sempre aperto durante tutta la seduta di lavoro e dove il discente copia gli indirizzi web dei siti visitati e aggiunge commenti sull'utilità del sito in questione. In un secondo momento pubblica questi appunti sul proprio weblog o sulla piattaforma didattica. Sarà allora leggibile per tutti i partecipanti del corso e per l'insegnante, in maniera che sia gli altri partecipanti, sia l'insegnante possano intervenire attivamente al lavoro individuale in atto. Inoltre in questo modo l'insegnante ha uno strumento di supervisione. Le critiche avanzate da parte dei partecipanti riguardavano tra l'altro l'effetto rallentante di questo procedimento: La navigazione risulta meno spontanea perché ogni passo utile viene verbalizzato.

\subsection{Tipologie di ricerche web}

Le sperimentazioni di didattica con supporti informatici svolti proponevano diversi tipi di compiti: Ricerche libere in internet e ricerche più strutturate, in cui l'input dell'insegnante non consisteva solo in una domanda posta ma anche in un catalogo di criteri formali da prendere in considerazione. I compiti più strutturati in assoluto sono i webquests ${ }^{5}$, definiti in una serie di "templates" prestabiliti, mentre i viaggi virtuali rappresentano una via di mezzo tra questi e le ricerche libere online.

\subsubsection{Webquests}

I webquests sono ricerche complesse sul Web, durante le quali gli apprendenti devono assolvere un compito che gli viene dato dall'insegnante sotto forma di uno scenario fittizio nel quale assumere un certo ruolo. È simile a scenari teatrali immaginari. Gli scenari proposti devono essere stimolanti per suscitare l'interesse e la motivazione nei discenti. Per esempio potrebbe essere delineato un progetto per un viaggio di studi, in cui i partecipanti hanno ognuno un proprio ruolo e un compito preciso da gestire per preparare una visita guidata. I discenti scelgono uno o più partners e insieme si assegnano dei ruoli o compiti all'interno dello scenario proposto. Da quel momento lavorano sempre in gruppo. Devono, nel ruolo a loro assegnato, procurarsi informazioni su internet per poter assolvere il compito, che consiste poi di regola in una comunicazione orale pubblica davanti a tutta la classe. A questo scopo, l'insegnante mette a disposizione dei singoli gruppi pochi link scelti che servono come punto di partenza per la loro navigazione in internet. Gli studenti navigano autonomamente, prendendo nota dei loro movimenti tra i vari siti aggiungendo immediatamente le loro impressioni o risultati. Poi i 
gruppi formulano insieme il risultato delle loro ricerche per risolvere il problema posto o per assolvere il compito. Presentano poi i loro risultati di fronte a un pubblico (la classe ipotizzata come comitiva di viaggio), per esempio in forma di una presentazione powerpoint.

Nell'anno accademico 2006/07 ho proposto un webquest a studenti del biennio in Germanistica. A prescindere dalle pure conoscenze linguistiche, gli studenti del biennio mostrano mediamente una maggiore disponibilità a nuove esperienze di apprendimento. Hanno di per sé più iniziativa personale e si mettono volentieri in gioco.

Il tema era la nuova musica poprock in Germania e il compito consisteva nell'assumere il ruolo di un agente di musica e presentare all'istituto Goethe di Napoli un complesso musicale o un musicista o un cantante da invitare ad un festival a Napoli di musica tedesca per i giovani italiani.

Gli studenti hanno poi riferito di aver impegnato per questo webquest complessivamente circa 8 ore, di cui 2 o 3 ore (circa un terzo) impegnate con la navigazione in internet con lettura di siti web in tedesco. Hanno dovuto affrontare un lessico specializzato del mondo della musica. Due terzi del tempo invece sono stati dedicati alla preparazione del prodotto finale, nel nostro caso nella presentazione Powerpoint. Le difficoltà erano la formulazione breve e concisa delle informazioni sulle diapositive powerpoint, adoperando i nuovi termini conosciuti, e naturalmente le questioni più strettamente tecniche: Per la stragrande maggioranza della classe l'uso del Powerpoint era una novità. Tutti i partecipanti hanno lavorato molto seriamente inventando strategie retoriche per assolvere il compito dato, mantenendo la finzione del futuro festival a Napoli per tutta la loro presentazione e prendendo il loro pubblico seriamente come giuria da convincere.

Hanno sperimentato tutte le tecniche dei media, inserendo testi, musica, video, cercando di far scorrere il testo animato parallelamente alla musica dell'audio, creando effetti ottici. Tutto questo anche con coinvolgimento di esterni, familiari, amici: una vera esperienza pratica e sociale che trascendeva lo spazio dell'aula sotto tanti punti di vista. ${ }^{6}$

Nel dibattito finale i partecipanti hanno dichiarato di aver, dal punto di vista strettamente linguistico, conosciuto un campo lessicale nuovo. Hanno considerato un' esperienza chiave dover parlare davanti ad un pubblico in una veste non proprio accademica, recitando un ruolo che trascendeva l'impostazione classica della relazione in classe. L'utilizzo di powerpoint veniva considerato da loro di grande importanza per la futura vita professionale.

La collaborazione con gli altri discenti, il lavoro in gruppo, è stato considerato dalla maggioranza un ostacolo nel procedere. Erano del parere che il webquest come tipologia di compito metteva troppi limiti sia per quanto riguarda il contenuto delle loro ricerche, sia per il procedimento del lavoro in gruppo e per la forma della presentazione finale. 
Lo scenario prestabilito, il ruolo conferito e la forma di powerpoint per la presentazione finale limitavano secondo loro oltremodo la loro creatività personale. Avevano investito, a loro parere, troppo tempo per la preparazione grafica e multimediale della loro presentazione powerpoint, mentre erano più interessati alla navigazione (semi-pilotata) in internet.

Se allora da parte dei discenti esiste il desiderio di autonomia accresciuta, questo tipo di attività, il webquest per l'appunto, resta relativamente statico e limitativo, e forse più consono alle esigenze delle scuole superiori, mentre a livello universitario, specie nel biennio magistrale, l'autocoscienza e l'autonomia accresciuta dei discenti permette anche attività di più vasta autonomia.

\subsubsection{Viaggi virtuali}

Più apprezzato dai discenti del biennio di Germanistica è stato un altro progetto, che lasciava molta più libertà: I viaggi virtuali. Il compito consisteva nello scrivere un diario di un viaggio virtuale (da non confondere con il diario del percorso di apprendimento) in una regione tedesca o austriaca di loro scelta. L'insegnante ha messo a disposizione una serie di link con informazioni su regioni e città. I discenti, navigando liberamente dai siti indicati, dovevano decidere individualmente e autonomamente la meta e il percorso del viaggio.

La programmazione del viaggio e il suo svolgimento con precise localizzazioni nel tempo e nello spazio era totalmente nelle mani degli studenti: Hanno consultato gli orari di treni ed aerei, scelto un albergo, fatto ricerche sui tempi di viaggio e le dimensioni topografiche della loro meta, e hanno visitato (sui singoli siti) ristoranti, musei, monumenti o altri luoghi di loro interesse, partecipato virtualmente a manifestazioni che si svolgevano in quel periodo ecc. La ricerca è stata documentata nel diario di apprendimento (online).

In seguito hanno steso un diario del loro viaggio virtuale individuale, nello stile di un diario: Scritto in prima persona con considerazioni spontanee, mescolando aspetti e prospettive personali con informazioni esterne. Hanno aggiunto delle foto, grafici e citazioni scaricate per illustrare gli avvenimenti del viaggio, sviluppando un percorso molto personale che poi è stato documentato in un documento word, sul blog o come pagina html. Diversamente dal webquest, i discenti hanno avuto qui l'occasione di scrivere quanto volevano in tedesco, descrivendo il loro viaggio virtuale. C'è chi è andato ben oltre lo stile letterario del diario e ha formulato parti di un romanzo, che trascendevano il compito dato.

Mentre nel webquest la difficoltà era di formulare titoli brevi e concisi per le schede di powerpoint, nel Reisetagebuch (diario di viaggio) si trattava di stendere un testo secondo precisi e affermati criteri stilistici. La sfida di uno stile ricco e specializzato era alta. 
Oltre al fatto nettamente linguistico, il viaggio vituale ha una potenzialità particolare: Navigare per posti di proprio interesse risulta di tutt'altra intensità psico-mentale che leggere una guida turistica o un qualsiasi altro libro sull'argomento. Una studentessa l'ha formulato così: In un libro abbiamo sempre i pensieri e le scelte di un altro, cioè dell'autore. In un viaggio virtuale attraverso navigazioni in internet restiamo noi protagonisti delle scelte. È nostro questo viaggio, e come tale viene virtualmente vissuto e depositato nella nostra memoria. È un esperienza che sta a metà strada tra leggere un libro e fare un viaggio reale. L'impatto per l'apprendimento è immediato e intenso.

Il ruolo dell'insegnante in questi scenari cambia fondamentalmente: Essendo sia la scelta del tema da parte degli studenti che il prodotto finale di impronta decisamente personale, talvolta quasi intimo, non è più prevedibile l'esito del compito. Sia i discenti sia l'insegnante si mettono molto in gioco. Si crea senza dubbio una atmosfera autentica di accresciuta fiducia. Il corso diventa una esperienza collettiva di partecipanti e insegnante che come tale apre nuove possibilità di comunicazione aiutando ad accrescere le motivazioni di apprendimento.

Il successo riscontrato con questo tipo di lavoro derivava forse anche dal fatto che si trattava di lavori prettamente individuali, che non richiedevano particolari interazioni in classe. Sotto questo punto di vista, il diario di viaggio risultava senza dubbio più tradizionale di un webquest e più consono alle tradizionali esperienze di apprendimento dei studenti.

\subsubsection{Libere ricerche web con la piattaforma didattica}

Nell'anno accademico 2008/09 ho tenuto per la prima volta un corso di lingua tedesca basato sulla piattaforma didattica Moodle. Il tema del semestre è stato accordato con gli studenti nel primo incontro. Hanno scelto il campo tematico delle minoranze in Germania, le migrazioni verso la Germania, i tedeschi emigrati in altre parti del mondo e le implicazioni storiche e sociali di questi fenomeni di migrazione e mobilità. Lacquisizione di sapere e di competenze si svolgeva attraverso compiti elaborati individualmente e nel collettivo. E anche la presentazione dei risultati era previsto, in seguito ad una serie iniziale di elaborati individuali, in forma collettiva, con pubblicazioni nei vari forum e wiki e con discussioni online collocati nell'apposito forum e nella chat. Tutte queste forme di comunicazione sono state automaticamente tracciate ed archiviate nel database di moodle. Durante gli incontri settimanali in aula si svolgevano i dibattiti in tedesco sui contenuti scoperti durante le sessioni individuali o collettive della settimana, e anche chiarimenti di problemi tecnici e attitudinali emersi durante la elaborazione collettiva dei wiki o nelle discussioni nei forum. Senza dubbio era il progetto intrapreso più 
propriamente "comunicativo", che richiedeva dai discenti una enorme apertura anche nei confronti degli altri.

I testi trovati dai discenti in internet (partendo da dei link multimediali, messi dall'insegnante sulla piattaforma) - in gran parte complessi trattati di argomento storico, sociale, istituzionale - risultavano linguisticamente e contenutisticamente molto impegnativi e solo una comprensione almeno approssimativa apriva la possibilità di ulteriori ricerche online.

Era sorprendente poter osservare che di fronte a queste fonti, i partecipanti avessero cercato quasi immediatamente di riconoscere la tendenza del testo e di acquisire una opinione a favore o contrastante. Formando ed eventualmente pronunciando di continuo il proprio parere si sono responsabilizzati di fronte all'oggetto dei loro studi.

Per i partecipanti del corso di fatto si trattava di una esperienza nuova di lettura e studio: Spinta dall'interesse personale la navigazione in internet veniva vissuta dagli studenti come esperienza molto intensa di lettura e di acquisizione del sapere. Raramente, così sostenevano, avrebbero letto con una tale intensità. Esternavano ogni settimana di aver imparato molto, seguendo le proprie curiosità e gli interessi individuali. Prevaleva tra di loro l'impressione di avere approfondito intensamente un argomento. La libertà di delineare il proprio percorso delle ricerche non veniva percepita come una restrizione o un impoverimento tematico in mancanza di un input pilotato dalla figura dell'insegnante, di un eventuale nuovo orizzonte indicato da una figura autorevole. Anzi, confermavano ad unisono che qualsiasi imposizione del pensiero lascerebbe in generale meno tracce nel pensiero individuale di una ricerca che parta dai propri saperi, dalle proprie curiosità, perplessità ed interessi.

Riconoscevano in corso d'opera i loro limiti nella collaborazione di gruppo, nell'elaborazione di risultati collettivi e nello scambio di pareri e informazioni con gli altri studenti. La loro libertà di opinione, di solito confinata alla sfera prettamente privata, è stata attivata in un contesto di studio, cioè in una sfera pubblica di tipo accademico-professionale: un percorso comunque intrapreso all'inizio con grande difficoltà. La propria responsabilizzazione nella elaborazione di un compito era per loro un'esperienza del tutto nuova e come tale molto proficua. Il puro fatto di mettere qualsiasi loro pensiero o risultato sulla piattaforma consultabile da tutti i partecipanti del corso li ha costretti ad aprirsi e agire esclusivamente nell'interesse della materia, prendendo coscienza di se stessi e delle proprie capacità. In questo senso questa sperimentazione si può considerare riuscita nell'accrescimento di un senso critico per le proprie conoscenze, il proprio agire e le proprie prospettive. L'insegnante diventa automaticamente una figura che principalmente coopera tenendosi in disparte e che rende i partecipanti consapevoli delle risorse a loro disposizione e dei loro reali punti di forza. 
La sperimentazione continua. Le difficoltà che i discenti stanno incontrando nel dover lavorare insieme in un vero teamwork, ma anche le potenzialità evidenziate nei loro tentativi di superare i propri limiti sia linguistici sia comportamentali, delineano la via da intraprendere nei prossimi progetti didattici per l'accrescimento dell'autonomia. L'impegno dei discenti nell' imparare le tecniche per una pacifica e proficua collaborazione con gli altri studenti, la creazione di un' autocoscienza su come e che cosa voler imparare aiuterà ad accrescere sia la maturità individuale sia la loro competenza sociale - premesse indispensabili per un percorso formativo di successo.

\section{Sito e bibliografia (scelta)}

L'apprendimento lungo tutto l'arco della vita: un diritto per tutti, 2002, Commissione Europea, Lussemburgo, Ufficio delle pubblicazioni ufficiali delle Comunità europee.

Calvani, A. 2004, Rete, comunità e conoscenza, Costruire e gestire dinamiche collaborative, Trento 2004.

Calvani, A. e Rotta, M. 1999, Comunicazione e apprendimento in Internet. Didattica costruttivistica in rete, Trento.

European Commission: Education \& Training <http://ec.europa.eu/education/programmes/newprog/index_en.html> (06/09).

Foerster, H. von 1995, Das Konstruieren einer Wirklichkeit, in Watzlawick, P. (a cura di.). Die erfundene Wirklichkeit. Wie wissen wir, was wir zu wissen glauben (9. Aufl.). München, Piper: 39-60.

Glasersfeld, E. von 1995, Einführung in den radikalen Konstruktivismus, in Watzlawick, P. (a cura.), Die erfundene Wirklichkeit. Wie wissen wir, was wir zu wissen glauben (9. Aufl.). München, Piper: 16-38.

Lodrini, T. (a cura di) 2002, Didattica costruttivista e ipermedia, IRRE Lombardia, Franco Angeli.

Mosel. S. 2005, Praktiken selbstgesteuerten Lernens anhand der Nutzung von webbasierten Personal-Publishing-Systemen, Diplomarbeit, Justus Liebig Universität Gießen Fachbereich 03: Sozial- und Kulturwissenschaften, Institut für Erziehungswissenschaft, Professur für Erwachsenenbildung, Gießen. $<$ http://weblog.plasticthinking.org/item/3779> (06/09).

Motivazione didattica $<\mathrm{http}$ //www.platon.it/progetti/ephilosophy/motivazione.htm> (06/09).

Rubenson, K., Lifelong learning. Una valutazione critica del Progetto Politico, online: <http://host.uniroma3.it/docenti/alberici/traduzione1.pdf $>(06 / 09)$. Watzlawick, P. (a cura di) 1995, Die erfundene Wirklichkeit. Wie wissen wir, was wir zu wissen glauben (9. Aufl.). München, Piper.

Werner Stangls Arbeitsblätter $<$ http://arbeitsblaetter.stangl-taller.at/LERNEN/ LerntheorienKonstruktive.shtml> (06/09).

Wolff, D. 1997, Lernstrategien: Ein Weg zu mehr Lernerautonomie, <http://www. ualberta.ca/ german/idv/wolff1.htm> (06/09). 
Note

${ }^{1}$ Il termine "life long learning", introdotto negli anni 50 nel mondo anglofono, riflette la necessità di essere capaci di adattamento alla evoluzione economica, tecnologica e sociale. Nel contesto della cosiddetta "globalizzazione", verso la fine del secolo il termine venne ripreso a proposito del nuovo postulato di flessibilità ed efficienza. http:// ec.europa.eu/education/programmes/newprog/index_en.html

${ }^{2}$ Il 1996 venne dichiarato l' Anno europeo dell'istruzione e della formazione lungo tutto l'arco della vita. Nel 2001 uscì il Memorandum sull'istruzione e la formazione permanente dell'Unione Europea (steso nell'ottobre 2000) e fino al 2006 vennero sviluppate strategie nazionali per la formazione permanente. A dicembre del 2006 entrò in vigore il nuovo programma europeo di formazione lungo tutto l'arco della vita. Nel maggio 2007 si è svolto a Berlino la cerimonia di inaugurazione di questo programma al quale viene fornito dal 2007 al 2013 un Budget complessivo di quasi 7 miliardi di Euro, per favorire lo scambio europeo di insegnanti e discenti di tutte le fasce di età e la collaborazione delle istituzioni di formazione.

${ }^{3}$ Il Portfolio Europeo delle Lingue è stato sviluppato dal 1998 al 2000 dal Consiglio d'Europa (Language Policy Division) a Strasburgo ed è stato diffuso durante l'anno Europeo delle Lingue 2001 come strumento per lo sviluppo di plurilinguismo e pluriculturalismo.

${ }^{4}$ Secondo la dottrina di Piaget le conoscenze non possono essere semplicemente "trasmesse" o "convogliate già pronte" ad un'altra persona (transfer di informazioni). Persino quando ci sembra di trasmettere informazioni con successo comunicandole a voce, se si potessero vedere in atto i processi mentali dell'interlocutore, si constaterebbe che questi "ricostruisce" una versione personale delle informazioni. L'approccio erogativo o ricettivo rischia sempre di opprimere e scoraggiare il partecipante con un inefficace e dannoso overloading di contenuti. http://www.platon.it/progetti/ephilosophy/ motivazione.htm

${ }^{5}$ Webquests sono stati sviluppati da Bernie Dodge e Tom March nel 1995 alla State University of San Diego. Intendono l'apprendimento come scoperta, ricerca, svolgimento di compiti, "inquiry-oriented activity" (Bernie Dodge) con l'uso del pensiero critico. "Il Webquest presuppone un nuovo modo di concepire l'attività del docente, che è chiamato a progettare e realizzare ambienti di apprendimento in cui gli allievi sono attivi e costruiscono i contenuti del loro sapere, lavorando in un contesto tecnologico di tipo costruttivista."

${ }^{6}$ Un altro corso, più recente, aveva come tema la storia delle regioni della Germania (Bundesländer), senza però creare uno scenario fittizio: In questo caso la ricerca Web e la presentazione dei risultati da parte dei discenti corrispondeva di più alle tradizioni didattiche universitarie. Tema era la formazione delle nuove regioni tedesche e le implicazioni storiche delle regioni con una lunga storia come entità politica-amministrativa, delle regioni storicamente contese con i popoli confinanti, delle regioni create ex nuovo da diverse realtà storiche, delle città-regioni e delle regioni con un passato come parti integranti del regno prussiano. Compito era di trovare informazioni attendibili in internet, facendo un profondo studio critico nei siti ufficiali di enti statali e regionali, e di associazioni storici, prima di creare con le informazioni ritenute valide e di qualità una presentazione powerpoint. 



\title{
MARCOS DE REFERENCIA PARA EL APRENDIZAJE PERSONAL
}

\author{
Pedro Pablo Sánchez-Villalón, Manuel Ortega, Asunción Sánchez-Villalón \\ Universidad de Castilla La Mancha
}

\section{Introducción}

Existe una creciente utilización de las plataformas de Gestión de Aprendizaje (LMS, acrónimo del inglés Learning Management Systems) promovida por el afán de estandarización que parece ser la tendencia común a seguir en eLearning. Sin embargo, algunos profesores e investigadores han criticado decididamente la aplicación de estas plataformas LMS (Britain \& Liber, 2004; Downes, 2006) ya que no parecen cumplir con los objetivos del nuevo concepto del aprendizaje mejorado por la tecnología, donde el aprendizaje constructivista está ocupando el lugar que tradicionalmente ha ocupado la enseñanza formalista, basada en la transmisión de contenidos, la transferencia del conocimiento. Por otra parte, se reconoce que la aplicación de la Tecnologías de Información y Comunicación (TIC) ofrece nuevas herramientas para la organización del aprendizaje. El uso de las plataformas LMS implica un gran avance, siendo las principales ventajas la accesibilidad a los contenidos y objetos de aprendizaje, y el seguimiento de las acciones del alumno. Sin embargo, son necesarios unos fundamentos más pedagógicos para que el uso de la tecnología sea más eficaz y tenga el éxito que la investigación reciente en tecnologías de la educación asegura (véase Jonassen et al, 1999; Britain y Liber, 2004). Esto conlleva un cambio de enfoque que pasa de centrarse en la organización de la enseñanza, propugnada por las plataformas LMS, a la concepción más pedagógica del aprendizaje propuesto en los nuevos entornos tecnológicos de aprendizaje. Lo que es más, se están desarrollando nuevos Entornos Personales de Aprendizaje (PLE, acrónimo del inglés Personal Learning Environments: Liber, 2005; Wilson, 2005) con el fin de que los usuarios puedan orientar ellos mismos la caótica experiencia que pueden tener al acceder a los diversos materiales para el aprendizaje en la Web.

Con esta evolución del eLearning se pueden aprovechar mejor las características del aprendizaje en Web: la amplia gama de recursos de comunicación que ofrece la Web, su ubicuidad, la capacidad de conexión, la facilidad para compartir recursos, la interacción y la colaboración, la 
accesibilidad, la flexibilidad y la libertad que proporciona. Sin embargo, todo esto nos podría llevar a hacernos a la falsa idea de que será fácil llegar al auto-aprendizaje, pero la realidad es que es más fácil caer en el caos de la información proporcionada por la Web. Si se aplican algunos principios básicos del aprendizaje constructivista que parece ser el más propiciado por el uso de las nuevas tecnologías, como los de la teoría de Zona de Desarrollo Próximo (Vygotzky, 1978), del andamiaje o scaffolding, la mediación, y la interiorización (internalization en inglés), el aprendizaje en Web debe, de alguna forma, estar basado en la organización, al menos en algún tipo de estructuración. Así, el uso de patrones o marcos sería necesario si el control de aprendizaje se va a poner en manos del alumno. Con este fin, y también considerando el papel de profesores, legisladores, editores de materiales de aprendizaje, etc., están apareciendo una serie de marcos de referencia en los nuevos sistemas oficiales de enseñanza, muchos propiciados por la aplicación de estas nuevas tecnologías, que pueden hacer que el aprendizaje en la Web pueda superar los objetivos establecidos por las instituciones de enseñanza y los límites impuestos por el uso de plataformas LMS controladas institucionalmente hacia sistemas de aprendizaje más controlados por el usuario.

Así, el entorno de eLearning que proponemos (AIOLE: acrónimo de Un Entorno Interactivo de Aprendizaje Online en inglés:An Interactive Online Learning Environment) está basado en el diseño de un espacio de aprendizaje con todos los recursos característicos de un entorno de aprendizaje personal, aplicando los estándares establecidos por una serie de marcos de aprendizaje, desde diferentes perspectivas: de organización, tecnológica y pedagógica.

\section{Marcos de Referencia}

Teniendo en cuenta en principio el uso y la aplicación de las TIC en la enseñanza, Johnson et al. (2006) han establecido un marco de referencia como modelo para los entornos personales de aprendizaje (PLE) desde la perspectiva tecnológica. Los alumnos pueden tomar el control de su propio aprendizaje, estableciendo sus objetivos de aprendizaje, reflexionando sobre ellos y sobre su proceso de aprendizaje, diseñan su aprendizaje con algunas herramientas disponibles, que, organizadas en un conjunto de actividades o tareas, se convierten en servicios online para el logro de esos objetivos. Estas herramientas consisten a menudo en recursos como fuentes RSS (Real Simple Syndication o de Sindicación de Contenidos), un calendario, capacidad de gestión de archivos, herramientas de comunicación, la implantación de la capacidad de escritura, y otros recursos (herramientas de idioma, edición de imágenes, el acceso a motores de búsqueda, etc.) Con el fin de convertirse en un modelo de referencia o marco, estas herramientas siguen una serie de pautas para 
convertirse en servicios (Fig. 1): patrones de la red con las fuentes de sindicación RSS para ofrecer acceso a contenidos y recursos de aprendizaje, patrones temporales para gestionar programas, patrones de conversación coherente para la comunicación, patrones de flujo de trabajo y los recursos para cargar y descargar archivos, imágenes y materiales, herramientas y actividades de lenguaje y la integración de una herramienta más eficaz para el aprendizaje personal en un modelo social: el uso de portfolios.

Fig. 1: El marco PLE como base de AIOLE

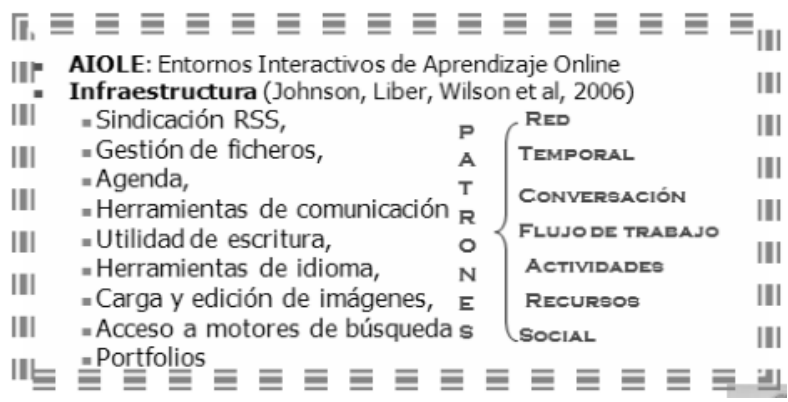

Otro marco puede establecerse a partir de las características de la Teoría de la Participación de Aprendizaje (Engagement Theory of Learning, Kearsley y Shneiderman, 1998) (Fig. 2), que puede ayudar en la modelización de este tipo de eLearning, con la participación de los alumnos en el co-diseño de actividades de aprendizaje con la ayuda o supervisión de la experiencia de un profesor.

Fig. 2: Engagement Theory of Learning (Kearsley and Shneiderman, 1998)

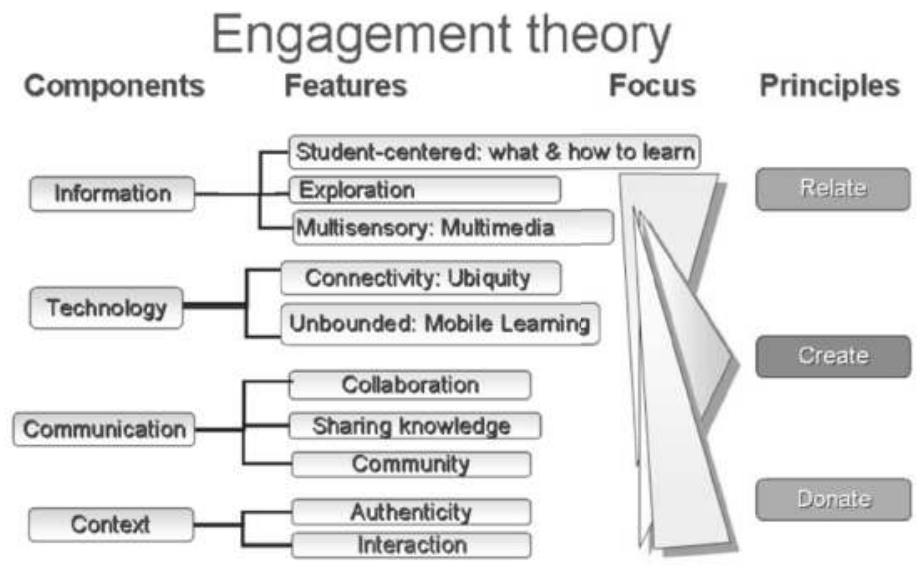

Relate (Relacionar información), Create (Crear utilizando la tecnología) Donate (Donar, publicar comunicándose) 
Anteriormente Shneiderman (1988) ya clasifica originalmente en tres los principios que rigen esta Teoría de la Participación: Relacionar, Crear, Donar. Para que el aprendizaje sea realmente motivador considera como punto de partida el trabajo en grupo de forma colaborativa en la etapa inicial de Relacionar para pasar a la de Crear proyectos ambiciosos (escribiendo informes, artículos, incluso poesía y música en grupo), lo que lleva a producir resultados realmente significativos fuera de la clase.

El término Relacionar puede verse como el aspecto cognitivo de la conexión en busca de información relacionada y de conocimiento compartido de cada usuario con el de los demás. Relacionar se puede ver como el elemento referencial de la información en el proceso del aprendizaje, en los tres sentidos: la que el usuario tiene, la que tienen los demás y la de las fuentes de información que se encuentran en el entorno de aprendizaje, ya sea el centro educativo físicamente hablando o el entorno online. Hace uso de los rasgos que identifican la información (centrándose en el alumno), la exploración de nueva información, y la cualidad multisensorial de la información; la condiciones tecnológicas y las utilidades de comunicación también influyen, pero un nivel secundario, en la creación de las condiciones para la colaboración.

En la etapa de Crear, intervienen en un grado mayor los componentes tecnológicos y algunas utilidades de comunicación (para colaborar y compartir el conocimiento). Aquí, además de los contenidos es importante enfatizar el conocimiento del idioma que se comparte. La capacidad de edición de un mensaje adecuado y correcto es lo que centra nuestro objetivo de aprendizaje de una lengua en este punto.

Por último, en la etapa de Donar, que consiste en la publicación o comunicación de ese conocimiento (generado por el intercambio de información con otras personas y de otras fuentes) lo que lo hace contextualmente auténtico, justificado y con una motivación extra para asimilar e interiorizar la información mediante la creación de un filtro de salida dirigido a los demás. En esta etapa final, tanto el ser parte de la comunidad de estudiantes (los destinatarios de la información), como la autenticidad de la información y la interacción serán las características más activas que influyen en el aprendizaje.

Así, con la participación en un entorno de aprendizaje online realmente motivador, podemos hacer un grupo que colabore en la búsqueda de información en la Web, en la edición de ideas compartidas y en escribir finalmente en la Web, siendo interesante para ello debatir con los demás, utilizando la fuente de información encontrada como motivación de entrada, y finalmente publicando el proyecto o el informe resultante en la Web. Este modelo se ha aplicado en este estudio para desarrollar recursos de aprendizaje online y crear entornos completos con características que posibiliten un aprendizaje realmente eficaz. 
Todo esto facilitará el diseño de tareas de aprendizaje si siguen un marco de un nivel superior, derivado de un marco de referencia más específico como punto de partida ya existente en ciertas disciplinas instrumentales, como es el aprendizaje de idiomas. Así, podemos establecer una forma práctica de aplicar los entornos personales (PLE) con estas nuevas características. Éste es el caso del Marco Común Europeo de Referencia para las Lenguas (CEFR, 2001), que establece la base pedagógica para el aprendizaje de idiomas bajo un enfoque orientado a la acción. Se compone de un conjunto de recursos (cognitivos, sociales y estratégicos) para adquirir el conocimiento de las competencias lingüísticas y comunicativas mediante la realización de una serie de actividades y estrategias en contextos o entornos de aprendizaje para manejar textos de forma tanto receptiva, como productiva y en definitiva interactiva, mediante el desarrollo de destrezas a practicar en tareas diseñadas pedagógicamente siguiendo un proceso de aprendizaje apropiado (Fig. 3).

En resumen, el CEFR (acrónimo del inglés Common European Framework of Reference) es un conjunto de descriptores y niveles que identifican las competencias lingüísticas y las destrezas que deben desarrollarse por medio de unas actividades y estrategias en una serie de tareas.

Fig. 3: El marco de referencia CEFR orientado a la acción

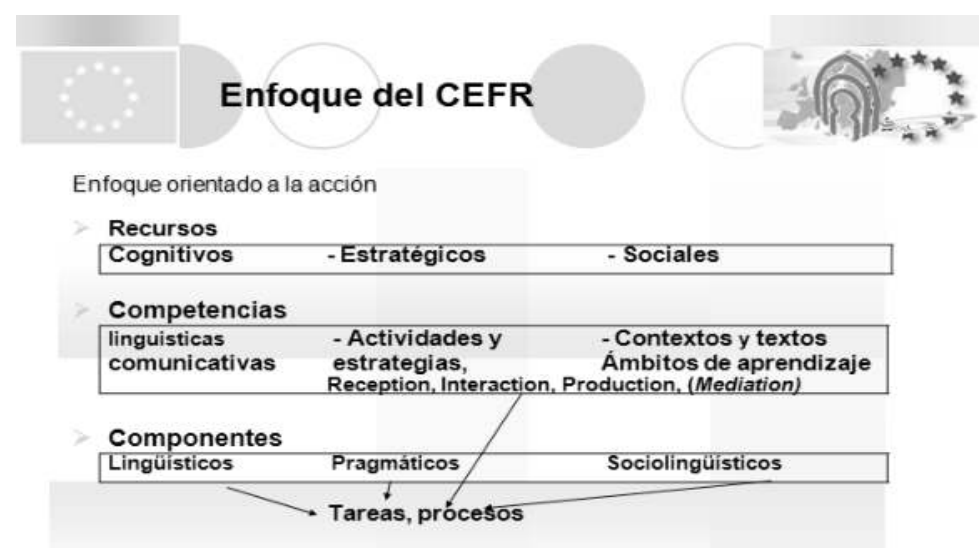

Los objetivos a alcanzar y los pasos a seguir para alcanzarlos se pueden encontrar de manera detallada en el Portfolio Europeo de las Lenguas (EPL, 2000), que tiene tres partes principales en su aplicación: el Pasaporte de Lenguas Europass, con una Tabla de Descriptores para la autoevaluación, la Biografía Lingüística, que promueve la reflexión sobre objetivos de aprendizaje específicos, y el Dossier, que muestra las actividades y acciones llevadas a cabo para alcanzarlos (Fig. 4). 
Fig. 4: Portfolio Europeo de las Lenguas

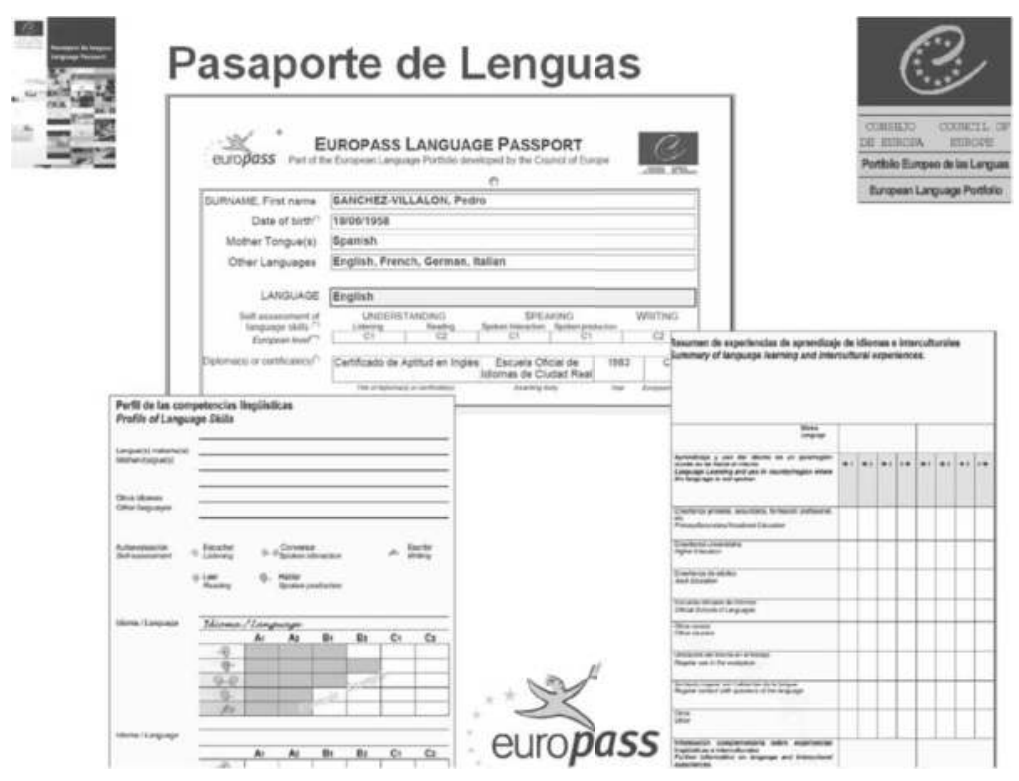

\section{AIOLE y el ePortfolio}

Dentro de estos marcos, la existencia de un entorno de aprendizaje online adecuado que reorganice todas las herramientas en servicios de aprendizaje facilitará la capacidad de ofrecer una plataforma de aprendizaje que se utilizará tanto como base formal como de forma personalizada e independiente para asegurar la realización de la educación permanente de manera fiable.

Si proporcionamos estas herramientas para ofrecer servicios de aprendizaje podemos aplicar con éxito los entornos de aprendizaje personalizado PLE, facilitando al alumno la posibilidad de diseñar su propio aprendizaje de las distintas destrezas, como la escritura a través de la Web, utilizando herramientas como los Blogs y los Wikis, la lectura, utilizando Webquests, y la escucha, con el uso de las Webcasts o Podcasts. Además, el acceso a la guía inicial de un tutor, a normas institucionales (del programa de la disciplina, del centro educativo, de entidades superiores) de forma escalonada - o scaffolding - y con sencillas técnicas de edición y de Copiar y Pegar ayudarán a diseñar y guiar su propio aprendizaje.

Hemos desarrollado el entorno de aprendizaje online AIOLE (Sánchez-Villalón y Ortega, 2007), como una solución intermedia que aspira a convertirse en un entorno PLE en el campo del aprendizaje de idiomas. Se trata de un conjunto de herramientas tecnológicas reunidas en el pla- 
Fig. 5: Edición del Europass (izquierda) y acceso al eDossier (derecha) con AIOLE

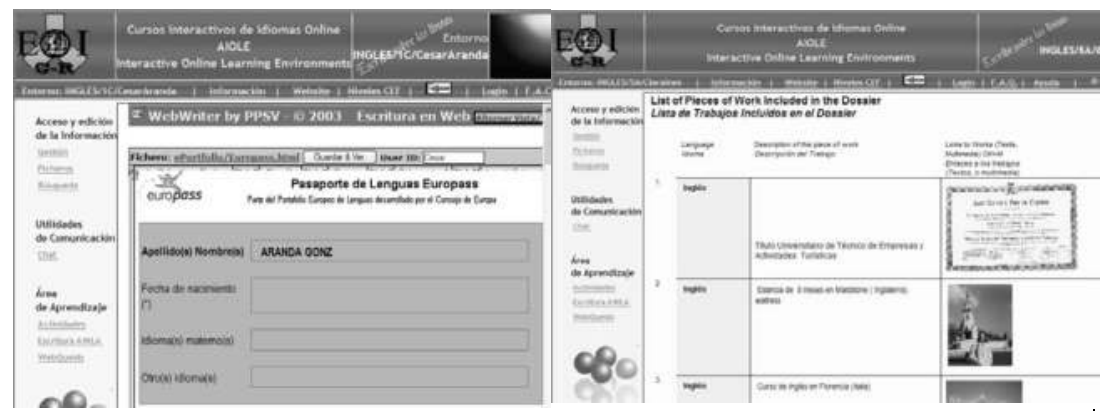

no pedagógico orientadas a servicios que el alumno puede utilizar para el aprendizaje independiente en la Web. Las herramientas ofrecen como principal innovación la capacidad para escribir en la Web - siguiendo la nueva tendencia de de la Web 2.0 de Escribir y Leer, la llamada Read / Write Web. Esto nos permite no sólo practicar la escritura, sino también diseñar actividades de aprendizaje en nuestro propio entorno. Con una fundamentación pedagógica, estas herramientas proporcionan acceso fácil a los descriptores y niveles CEFR en forma de servicios y, usando las plantillas oficiales del Europass, la edición fácil del ePortfolio. Las actividades son diseñadas aquí por los propios alumnos con la posibilidad de utilizar una serie de plantillas y aplicaciones basadas en tecnologías Web. AIOLE ofrece con estas herramientas y con el diseño de aprendizaje una solución intermedia, ya que los alumnos necesitan alguna orientación de un tutor en sus etapas iniciales para e-Learning. Más tarde, serán capaces de seguir aprendiendo en el mismo escenario, sin un tutor, en un entorno cada vez más independiente, de manera informal, lo que les llevará a la formación permanente.

AIOLE tiene algunas rasgos que son característicos de las plataformas LMS:

- Administración de archivos (con capacidad de acceso y carga de información en forma de ficheros),

- Recursos para la comunicación (como Chat / Foros),

- Organizadores (con una Agenda)

- La posibilidad de realizar el seguimiento de las acciones de los usuarios (sistema de seguimiento o tracking)

También ofrece algunas características de los entornos de aprendizaje online (OLE) y entornos de aprendizaje personalizados (PLE) para ayudar a los estudiantes a tomar el control de la gestión de su propio aprendizaje:

- Uso de WebWriter para la edición de tareas y actividades en ficheros $\mathrm{rtf} / \mathrm{html}$ en la Web, 
- AWLA para practicar la escritura, la comprensión oral o escucha y para la gestión multimedia,

- y la edición y gestión del ePortfolio (Fig. 5), donde el alumno puede diseñar la evolución de su propio aprendizaje, su CV o su pasaporte de idiomas, y su eDossier (Sanchez-Villalon et al, 2007), con acceso a los certificados e imágenes escaneadas, o archivos de audio y vídeo necesarios para mostrar la evidencia de su aprendizaje.

\section{Conclusiones}

El uso de las herramientas TIC ha implicado un cambio destacado partiendo de la enseñanza como transferencia de conocimientos controlada por la institución educativa y el profesor a la construcción del conocimiento más activa controlada por los alumnos. Existe la posible ventaja de establecer un proceso de aprendizaje guiado inicialmente por tecnología hacia una mayor independencia aplicando los recursos disponibles proporcionados por la Web. Las factores más relevantes que han hecho evolucionar el aprendizaje hacia el eLearning, como son la distribución de contenidos (cuyo intento de estandarización ha dado lugar a los llamados objetos de aprendizaje), la interacción (como culminación de la teoría de la Participación o Engagement) y el diseño del aprendizaje (el factor clave para alcanzar el objetivo último de aprendizaje a lo largo de toda la vida o Lifelong Learning), permiten establecer una clasificación de los entornos de aprendizaje online en Learning Management Systems (LMS), Online Learning Environments (OLE) y Personal Learning Environments (PLE).

También se ha producido un cambio en los fundamentos pedagógicos: proporcionar el acceso a nuevos tipos de información, a nuevos contenidos, nuevas disciplinas en la Web, a la capacidad de modificar el contenido y el diseño de aprendizaje con acceso a recursos de la Web 2.0, a nuevas modalidades de aprendizaje basadas en marcos, ayudando al alumno a diseñar su aprendizaje a través de las tareas de aprendizaje, con el acceso a entornos PLE regidos por las normas CEFR, en lo que al aprendizaje de idiomas se refiere.

Estos entornos interactivos permiten diseñar tareas o actividades de aprendizaje, realizar un seguimiento de las acciones del usuario, adaptar el escenario según la evolución del aprendizaje del alumno y ofrecer alternativas adicionales en su proceso de aprendizaje. Es la experiencia del aprendizaje lo que importa en estos nuevos entornos interactivos. Las actividades de aprendizaje quedan registradas en el entorno ayudando en el proceso de desarrollo el conocimiento que requiere una disciplina lingüística, a la vez que servir de experiencia para el uso de recursos disponibles para el aprendizaje permanente. Estas experiencias lingüís- 
ticas junto las realizadas en entornos clásicos y formales (académicos) y en entornos reales de aprendizaje informal las que conjuntamente proporcionan información y evidencia de las capacidades lingüísticas de los usuarios. El uso de marcos y modelos permite reflexionar sobre esas experiencias lingüísticas y colocar referencia a ellas como evidencia de sus capacidades lingüísticas por medio de un recurso digital para el Europass, la Biografía Lingüística y en última instancia el eDossier, los componentes del Portfolio en una versión digital.

\section{Referencias}

Britain, S. and Liber, O., (2004). A Framework for the Pedagogical Evaluation of eLearning Environments. Bolton Institute. <http://www.cetis.ac.uk/ members/pedagogy/files/4thMeet_framework/VLEfullReport>(03/09).

CEFR (2001). Common European Framework of Reference for Language Learning, Teaching and Assessment. Council of Europe <http://www.coe.int/t/dg4/ linguistic/CADRE_EN.asp>(03/09).

Downes, S: (2006). The Students Own Education, National Research Council Canada.

EPL, 2000. European Language Portfolio (Principles and guidelines, Guides) $<$ http://www.coe.int/portfolio>(03/09).

Johnson, M., Liber, O., Wilson, S., Sharples, P., Milligan, C., Beauvoir, Ph., (2006) "Mapping the future: The personal Learning environment reference model and emerging technology". In Whitelock, D. \& Wheeler, S. (Eds). The Next Generation. Research Proceedings of the 13th Association for Learning Technology Conference (ALT-C 2006). Heriot-Watt University: Edinburgh, UK.

Jonassen, D.H., K. L. Peck, Wilson, B. G. (1999). Learning with Technology. A Constructivist Perspective. New Jersey: Prentice Hall.

Kearsley, G., Shneiderman, B. (1998) "Engagement theory: A framework for technology-based teaching and learning", Educational Technology 38, 5 (September-October 1998), pp. 20-23.

Liber, O. (2005) Personal Learning Environments project, <http://www.elearning.ac.uk/subjects/PLE $>(03 / 09)$.

Sánchez-Villalón, P.P., Ortega, M. (2007) "AWLA and AIOLE for Personal Learning Environments". In International Journal of Continuing Engineering Education and Life-Long Learning (IJCEELL), InderScience Publishers Ltd.

Sánchez-Villalón, P.P., Ortega, M., Sánchez-Villalón, A. (2007) "AIOLE and AWLA application to e-Learning 2.0: an ePortfolio with an eDossier". In Micromedia and Corporate Learning Proceedings of the 3rd International Microlearning 2007 Conference, M. Lindner, P. A. Bruck (Eds.), Innsbruck University Press: Innsbruck, Austria.

Shneiderman, B. (1988), "Relate Create Donate: An educational philosophy for the cyber-generation", Computers \& Education 31, 1, 25-39 (DCL-ben). 
Vygotsky, L. S. (1978). Mind in Society: The Development of Higher Psychological Processes. (Translated by Cole, M., M. Lopez-Morillas, A. R Luria, J.Wertsch,. Original work published 1930). Cambridge, MA: Harvard University Press.

Wilson, S. (2005). The PLE debate begins. <http://www.cetis.ac.uk/members/ scott/blogview?entry=20051126183704>(03/09). 


\title{
EL AULA VIRTUAL DE ESPAÑOL (AVE): UN ENTORNO PARA EL APRENDIZAJE AUTÓNOMO
}

\author{
María del Pilar Hernández Mercedes \\ Instituto Cervantes de Nápoles
}

\section{Introducción}

El Instituto Cervantes, ente oficial español para la difusión de la lengua y la cultura española e hispanoamericana, creado en 1991, ha mostrado un gran interés desde sus albores, en plena revolución tecnológica, por las Tecnologías de la Información y la Comunicación (TIC) y la importancia que reviste su integración en la sociedad de hoy.

El Instituto Cervantes en su apuesta por integrar las TIC en la enseñanza/aprendizaje del español y con el deseo de cubrir así un espacio vacío, al no existir hasta ese momento ningún curso institucional de español en Internet, ha diseñado el AVE (Aula Virtual de Español), actualmente, uno de sus productos estrella.

Este recurso didáctico, basado en el principio de la accesibilidad, vio la luz en el año 2000 con el nivel inicial (compuesto por cuatro cursos), y se ha integrado progresivamente, quedando completado en octubre de 2005 con los cuatro cursos que componen el nivel superior, sumando un total de dieciséis cursos divididos en cuatro niveles (Inicial, Intermedio, Avanzado y Superior).

De esta manera, se ha visto cumplido el objetivo de diseñar un producto de calidad, tanto desde el punto de vista metodológico, como desde el punto de vista técnico, y a la vez, ofrecer un producto "abierto", cuya utilización no se restringe al uso exclusivo en el marco del Instituto Cervantes y de sus centros repartidos por todo el mundo, sino al servicio también de la demanda formativa de las más variadas instituciones.

\section{2. ¿Qué es exactamente el ave?}

Se trata de un entorno virtual autónomo de aprendizaje creado específicamente para la enseñanza del español (todos los elementos que incorpora, el diseño, la estructura, etc. han sido concebidos desde un punto de vista didáctico y metodológico específico). No estamos, por lo tanto, ni 
sólo ante un variado compendio de material didáctico, ni ante una "estructura" utilizable para el aprendizaje de otras materias, sino ante un entorno que consta de una plataforma con todos los espacios, materiales y herramientas necesarios para el aprendizaje del español y su gestión.

Pero, ¿qué se entiende por Entorno Virtual de Aprendizaje?1

Se trata de "una aplicación informática diseñada para facilitar la comunicación pedagógica entre los participantes en un proceso educativo, sea este completamente a distancia, presencial o de una naturaleza mixta que combine ambas modalidades en diversa proporciones" (Adell, Castellet y Gumbau, 2004).

Según estos mismos autores, sus cuatro principales usos son:

- Distribuir materiales educativos en formato digital.

- Poner en contacto a estudiantes y tutores organizando discusiones y debates en línea.

- Utilizar contenidos pertinentes de la red.

- Posibilitar la contribución de expertos a los debates y charlas.

A los que Abad Castelló (2006) viene a añadir el de seguimiento y evaluación del progreso de los estudiantes por parte del sistema.

Un EVA se caracteriza por contar con tres tipos fundamentales de herramientas: de comunicación (síncronas y/o asíncronas), de gestión de los materiales educativos y de gestión de los alumnos (también su seguimiento y evaluación).

El AVE, caracterizado por su flexibilidad y por estar en constante actualización (incorporando las novedades tecnológicas que surgen de manera incesante en el mercado, los cambios en la enseñanza en línea y en el mundo que se representa en las actividades), presenta un diseño en módulos independientes y una estructura fragmentada. Por esta razón, aunque la idea inicial era el uso como entorno autónomo de aprendizaje -con todos los recursos y herramientas necesarios para $\mathrm{ello}^{2}$ - su estructura permite integrar los materiales didácticos como un recurso de apoyo de cursos presenciales de cualquier institución.

De acuerdo con las principales líneas de explotación de los materiales en Internet, el AVE es un recurso para la enseñanza de E/LE que permite diferentes usos. Para ello, se han concebido diferentes modalidades de explotación (en cursos a distancia, en cursos semi-presenciales, como base de cursos presenciales, y como recurso de aprendizaje) que suponen un acceso más o menos restringido de los alumnos a los espacios del AVE, contando en determinadas ocasiones con un tutor a distancia y/o con un sistema de seguimiento.

Al tratarse de un sistema integrado (combinación de $\mathrm{MDM}^{3}$, herramientas de comunicación y de gestión de la actividad docente) de enorme flexibilidad, podemos hablar de distintas modalidades de uso y de explotación, que analizaremos más adelante. 
Antes de cerrar este apartado, insisto en una de las características esenciales del AVE: el haber sido diseñado exclusivamente para la enseñanza del español,y esto lo hace único al tratarse de un entorno propio.Todo está pensado y diseñado pensando en los cursos de español que a través de él se ofrecen.

\section{Estructura}

El material didáctico del AVE se divide, según el Plan Curricular del Instituto Cervantes, en 4 niveles (Inicial, Intermedio, Avanzado y Superior). El nivel inicial corresponde a los niveles A1 y A2 del Marco Común

de Referencia Europeo, el Intermedio al B1 del Marco, el Avanzado al B2 del Marco y el Superior al C1 del Marco.

Cada uno de los niveles del AVE, a su vez, se divide en cuatro cursos. Un curso corresponde a entre 40 y 60 horas de trabajo del alumno, y puede hacerse -a un ritmo medio- en unas 12 semanas (en el IC de Nápoles, las semanas por licencia son, en cambio, siempre 16, de manera que los alumnos cuenten con más tiempo a su disposición para poder realizar todos los ejercicios, mejorarlos, realizar los materiales complementarios que más les interesen, repasar, rehacer, etc.).

En cada tema, encontramos 9 sesiones de trabajo, una Tarea Final de tipo colaborativo (en la que se ponen en práctica los conocimientos adquiridos a lo largo de las sesiones), y una autoevaluación, llamada Aduana, de la que el alumno, a través del sistema, obtiene el porcentaje de aciertos pero no la solución. La finalidad de este test de evaluación automática es que el alumno, dado que es informado sobre su aprovechamiento en el tema, tome conciencia de los conocimientos adquiridos y de sus carencias y, si es necesario, repase o repita alguna de las sesiones. La historia gráfica interactiva o aventura gráfica que encontramos al final, diferente para cada nivel, sirve para trabajar los contenidos del tema de una forma más lúdica.

Todo lo anterior constituiría la secuencia didáctica principal en la que se trabajan los contenidos curriculares de forma secuenciada y atendiendo a las cuatro destrezas, a lo que se vendrían a sumar los materiales complementarios de realización libre (según deseo del alumno o designación del tutor) que sirven para repasar o practicar ulteriormente algunos contenidos o para trabajar de manera más específica con alguna de las destrezas.

Todos los materiales están organizados en una estructura sencilla y muy fácil de seguir.

\section{El entorno de aprendizaje}

El entorno de aprendizaje del AVE (http://ave.cvc.cervantes.es) es el espacio en el que se accede a los distintos ambientes del Aula Virtual 
(alumno, tutor y, aparte, administración), cada uno con las herramientas específicas necesarias para su buen funcionamiento.

\subsection{Entorno de aprendizaje del alumno}

La Sala de Estudio, espacio al que el alumno accede cuando introduce sus claves, es el lugar donde éste encuentra todo el material didáctico multimedia (es decir, todas las actividades y propuestas que configuran cada curso).

Además tiene el acceso a:

- sala de comunicaciones, donde se encuentran las herramientas de comunicación que utilizará para entrar en contacto con el tutor o los compañeros, eligiendo uno u otro según el tipo de comunicación necesaria - chat, foro, correo electrónico, calendario y tablón de anuncios

- seguimiento y evaluación, donde el alumno puede seguir sus progresos

- calendario

- banco de recursos, donde puede encontrar tanto actividades como enlaces propuestos por el tutor

- tablón de anuncios

El entorno, caracterizado por una gran flexibilidad, permite, tanto la total autonomía por parte de sus usuarios, como que estos formen parte de un grupo de trabajo colaborativo y cuenten con el apoyo de un tutor que les guíe en su aprendizaje, lo cual no excluye, es más alimenta, que se desarrollen estrategias de aprendizaje ("aprender a aprender") que den más autonomía al estudiante al hacerlo más consciente de su propio proceso de aprendizaje.

\subsection{Entorno de gestión docente}

Se accede a través de la misma dirección que el anterior con una clave de tutor. En este espacio, encontramos:

- los materiales de todos los niveles

- herramientas para comunicarse con los alumnos, ver su seguimiento y evaluarlos

- espacios de comunicación con los otros tutores, con los que compartir experiencias y materiales a través del Banco de recursos, los foros, el correo electrónico, etc

- curso de formación de tutores, recurso siempre accesible, que sirve de guía para el uso y explotación del AVE. En él, el profesor además de poder formarse como tutor a distancia, aprenderá a integrar este nuevo recurso en sus clases regulares 


\subsection{Entorno de gestión administrativa}

En la dirección http://admin.ave.cervantes.es, de acceso restringido (destinado a Jefes de estudios y administrativos de los centros), encontramos el espacio propio para realizar todas las tareas administrativas del AVE (dar altas, bajas a tutores y alumnos, crear grupos, activarlos, cerrarlos, llevar históricos, etc.).

\subsection{Portal informativo: http://ave.cervantes.es}

A todos estos entornos se les viene a sumar el portal informativo del AVE, de acceso libre y en cinco lenguas, cuyo objetivo es difundir el AVE a través de la red, proporcionar a los interesados información general (metodología, modalidades, tutores, alumnado, requisitos técnicos del ordenador, etc.). Cuenta además con una demo de los cursos y con una prueba de nivel orientativa (para comprobar el nivel de español de forma automática). Se incluye también información sobre todos los centros que ofrecen el AVE con los contactos correspondientes y el acceso a un servicio informativo (infoave@cervantes.es) donde dirigirse para resolver todas las dudas y hallar respuesta preguntas que hayan ido planteando.

En la página institucional del Instituto Cervantes y en la del Centro Virtual Cervantes hay también enlaces al portal del AVE. De esta forma, todas las instituciones que ofrecen el AVE cuentan con el apoyo de difusión que suponen unas páginas web visitadas por miles de personas cada día.

Una de las últimas novedades dentro del "Aula de español" del CVC, es la AVETECA, espacio en el que se ofrece semanalmente una actividad del AVE, constituida normalmente por varias pantallas y una ficha explicativa para el profesor y otra para los alumnos. Cada actividad, tras una semana, pasa a formar parte del archivo de la AVETECA, que es muy fácilmente consultable a través de un listado de funciones. Hasta el momento encontramos numerosas actividades del nivel inicial.

En esta sección, al ofrecerse materiales de libre acceso para docentes y discentes, queda patente la finalidad por parte del Instituto Cervantes de dar a conocer y de difundir en lo posible el AVE.

\section{Materiales didácticos}

Tras haber analizado, si bien someramente, los diferentes entornos y espacios del y para el AVE, pasemos ahora a ocuparnos del material didáctico y, más concretamente, del material didáctico multimedia (MDM), componente rey de los cursos de español. 
Es sabido que, en la formación a distancia, los materiales adquieren una especial relevancia, puesto que en este ámbito el foco está completamente dirigido al alumno y el papel del tutor pasa a ser fundamentalmente de guía y de facilitador del proceso de aprendizaje. Por ello, los materiales tienen que reunir una serie de características para permitir y facilitar el trabajo autónomo sin que sea necesario un apoyo constante, con ayudas ofrecidas por el sistema para resolver las posibles dudas que pudieran surgirles a los alumnos. Tanto el contenido como el diseño tienen que estar orientados a dar respuesta a los diferentes estilos y ritmos de aprendizaje y el alumno ha de contar con los medios adecuados para poder tener una experiencia de aprendizaje positiva.

Los materiales didácticos del AVE cuentan con un enorme componente multimedia (vídeo, imágenes, animaciones, textos, hipertextos, etc.), cuyas ventajas más inmediatas -la multiplicidad de medios, la opcionalidad, la flexibilidad y la interactividad- son bien aprovechadas. Además, y gracias al empleo de distintos medios (vídeo, audio, imagen, animación...) activan diferentes tipos de mecanismos cognitivos.

Los materiales se dividen en dos grandes grupos, con una estructura muy fácil de seguir: la SECUENCIA DIDÁCTICA PRINCIPAL, de la que ya nos hemos ocupado en la página 5 y los MATERIALES COMPLEMENTARIOS, estructurados en:

- Talleres, para trabajar los contenidos lingüísticos.

- Sonidos y letras, para la fonética y la ortografía.

- Otras rutas, para trabajar estrategias de aprendizaje con el fin de proporcionar al alumno de mayor autonomía en su proceso de aprendizaje.

- Excursiones, en que se sugieren al alumno visitas guiadas a determinadas páginas web en español, reales, para buscar información concreta con la que luego se trabaja; En este apartado se pone de relieve el uso de la red como fuente de materiales auténticos al proponérseles direcciones de Internet para profundizar en el área temática que estén tratando.

- Fichas, donde se recogen de forma sistemática los contenidos lingüísticos trabajados (funcionales, gramaticales, etc..).

El alumno cuenta también con un Glosario con definiciones, ilustraciones y ejemplos de uso de las palabras del curso.

Estos materiales, que sirven para repasar, profundizar o practicar más algunos de los contenidos o trabajar destrezas concretas, serán realizados por los alumnos que lo consideren oportuno o por indicación del tutor, pudiendo trazarse itinerarios completamente personalizados. Revisten, por lo tanto, gran relevancia a la hora de atender necesidades especiales y diferentes ritmos y estilos de aprendizaje.

Las actividades del AVE se basan en la activación de los conocimientos previos del alumno y dan respuesta a los distintos estilos de 
aprendizaje. El aprendizaje en el AVE siempre es significativo (el énfasis recae en el significado más que en la forma), como corresponde a un enfoque comunicativo, estableciéndose como objetivo la comunicación en la lengua meta sin descuidar ninguno de los aspectos que la hacen posible.

Estructuralmente, la organización responde al enfoque por tareas. A través de las diferentes sesiones, el alumno adquiere conocimientos (a todos lo niveles) necesarios para realizar una tarea final de carácter colaborativo (que implica comunicarse con otros compañeros).

\section{Modalidades de explotación}

Como podemos leer en el folleto informativo institucional del AVE y en el portal dedicado a éste (http://ave.cervantes.es), "en función de las necesidades y el perfil de los alumnos, se pueden emplear distintos modos de explotación:

1. Presencial: como base del programa del curso o material de apoyo.

2. Semipresencial: con clases presenciales en número y frecuencia variables.

3. A distancia: con un grupo de aprendizaje y un tutor, o de forma autónoma.

\section{Presencial}

Los alumnos trabajan en el aula multimedia, en el laboratorio o en el aula presencial con ordenador y proyector. Se puede elegir el itinerario marcado por el propio AVE, constituyéndose este así en base de la programación, o utilizar diferentes actividades para integrar el propio programa según las necesidades que se vayan planteando o según un plan preestablecido por el docente o por el centro. El uso del AVE como material de apoyo, refuerzo y consolidación se está difundiendo mucho en instituciones educativas ajenas al Instituto Cervantes, ya sea con licencias de tipo AULA, que permiten el acceso a todos los cursos de todos los niveles, ya sea con licencias regulares de acceso a un curso concreto.

\section{Semipresencial}

La estructura y características del AVE se revelan idóneos para una enseñanza combinada. Como indican los especialistas del AVE del departamento de Tecnología y Proyectos lingüísticos del Instituto Cervantes: (http://www.formatex.org/micte2005/148.pdf, página: 3): "la programación mixta de un curso semipresencial aprovecha y potencia todo el trabajo que el estudiante necesita hacer de forma autónoma para que se produz- 
ca la adquisición con el aprovechamiento de las horas de asistencia a clase para dedicarse a la producción e interacción oral y al esclarecimiento de otros aspectos de la secuencia de aprendizaje que necesitan de la intervención del autor". La institución establecerá cuántas y de qué manera distribuir las horas presenciales asignadas a cada curso. Estas últimas normalmente se reservan sobre todo para el desarrollo de la destreza oral, la puesta en común de opiniones y la resolución de dudas de manera más directa y aprovechando las ventajas de hallarse en un grupo.

\section{A distancia}

Encontramos diferentes posibilidades:

A distancia con tutor (matriculado)

El estudiante, en este caso miembro de un grupo, realiza el itinerario trazado por el AVE con la ayuda y apoyo constante de un tutor que le guiará, se ocupará de la organización de las actividades cooperativas, gestionará las herramientas de comunicación para obtener de ellas los mayores beneficios en términos de aprendizaje efectivo de la lengua, entre otras funciones.

Nada resta libertad al estudiante, todo en el entorno le llevará a ir adquiriendo una mayor autonomía y toma de conciencia del propio proceso de aprendizaje.

A distancia con tutor (registrado)

El estudiante obtiene unas claves de acceso para entrar libremente y de manera completamente autónoma (no forma parte de un grupo, ni cuenta con el apoyo de un tutor) en el curso AVE en el que se haya matriculado durante un tiempo estipulado (entre 12 y 16 semanas, dependiendo de cada centro). Podrá seguir el itinerario que marca el propio AVE y realizar el número de actividades que desee o trazar su propio recorrido según sus necesidades específicas.

Su trabajo no será sometido a ningún control "humano". Sólo contará con el seguimiento realizado por el sistema para orientarse sobre cómo está realizando las diferentes actividades. A la máxima libertad que supone esta modalidad, se le opone la facilidad de que el estudiante "se pierda", es decir, se desmotive (si no es muy fuerte la motivación que le ha llevado a elegir esta modalidad) puesto que no es fácil realizar un itinerario de aprendizaje en total autonomía y sin contar más que con apoyos "del sistema".

A las modalidades de explotación clásicas del AVE, con las variantes que puedan registrarse en su uso en las diferentes instituciones educativas y en el propio Instituto Cervantes, viene a sumarse, dentro de los cursos $A$ distancia sin tutor (registrado), el apenas creado Supervisado ${ }^{4}$. 
En este caso, el alumno gestiona de manera autónoma el tiempo (16 semanas) y los materiales según su ritmo de aprendizaje, necesidades concretas y posibilidades de acceso, pudiendo realizar un itinerario totalmente personalizado, trazado por el propio usuario.

Para los materiales de la secuencia básica no hay opcionalidad, puesto que los deberá realizar en su totalidad (a excepción de las actividades colaborativas y las tareas finales, pues estos alumnos no forman parte de un grupo y no tienen modo de interactuar con otros estudiantes para poder realizar esta tipología de propuestas). Para poder aspirar a la obtención de un certificado al finalizar el curso, la opcionalidad queda reservada para los materiales complementarios y la aventura gráfica. El alumno, y como características específicas de esta modalidad, obtendrá la supervisión, el control final y la elaboración de un informe, por parte de un "supervisor", a la luz de los resultados que ofrece el apartado seguimiento y evaluación sobre el alumno. Recibirá, además, un certificado en caso de resultado positivo. ${ }^{5}$

Por lo tanto, este nuevo recurso didáctico puede emplearse como herramienta autónoma para el aprendizaje por Internet (enseñanza a distancia) o integrarse en propuestas docentes ya consolidadas (enseñanza presencial y semipresencial) con total flexibilidad.

Esta misma característica permite, a su vez, que frente al recorrido linear que el estudiante puede seguir con o sin la ayuda de un profesor o tutor, exista la posibilidad de crear y aplicar rutas no lineares trazadas por el profesor o por los propios estudiantes (aunque generalmente manifiestan preferencia por hacer trayectorias establecidas) según sus necesidades específicas (es decir, se pueden "diseñar" recorridos personalizados).

Para esto se revelan de inestimable ayuda las programaciones que, como material complementario, acompañan al AVE, ya que se podría establecer la ruta eligiendo materiales del "cuerpo central" y/o fundiéndolos los materiales complementarios dispuestos en desplegables de fácil acceso y consulta.

\section{Autonomía de aprendizaje y Aprendizaje autónomo}

Como hemos ido viendo, todo en el AVE está diseñado para fomentar un aprendizaje personalizado y autónomo y para desarrollar, independientemente de la modalidad de explotación que se elija, la autonomía de aprendizaje.

Vamos a detenerlos ahora en algunos de los principios y características (finalizados a la consecución de la autonomía) que podemos detectar en el AVE. 


\subsection{Promover y desarrollar estrategias de aprendizaje}

Los diferentes tipos de actividades ponen en marcha la activación de las más variadas estrategias: inferir significados, deducir información, comprensión global, comprensión específica, lectura selectiva, etc. Basta pasar revista a los materiales que ofrece el AVE para obtener ejemplos de todas las tipologías.

La formulación de las indicaciones explicita bastante qué estrategias de aprendizaje se van a desarrollar en cada una de las actividades, facilitando que el estudiante sea consciente de ello.

7.2 Ofrecer ayuda y retroalimentación al estudiante de modo que adquiera independencia de la figura del profesor o tutor

El alumno, además de poderse servir de manera autónoma de toda una red de ayudas ${ }^{6}$, podrá obtener la información también de los propios compañeros (a través de herramientas como la chat, los foros o incluso el correo electrónico).

El AVE cuenta con un completo sistema de seguimiento y evaluación al que acceden no sólo los tutores, sino también los alumnos que así sabrán con exactitud cómo están aprendiendo y podrán actuar en consecuencia.

\subsection{Variedad en la tipología de las actividades propuestas}

De esta manera el estudiante puede potenciar sus propios intereses, aumentando así su motivación (tanto cuando realiza las actividades de forma linear y todas, como cuando elige en qué propuestas centrarse). En los materiales del AVE hay, además, una enorme diversidad de formas de interacción con el sistema: arrastrar, pulsar, relacionar, grabar, rellenar cajas de texto, diversos juegos, etc. Esta variedad, que atiende a diferentes estilos de aprendizaje, es un elemento más para lograr la motivación del estudiante.

Como ya dijimos al tratar los MDM, con el AVE "se aprovechan las ventajas más inmediatas de los MDM (materiales didácticos multimedia) como son la multiplicidad de medios, la opcionalidad y flexibilidad, y la interactividad, y se permite la puesta en marcha de diferentes tipos de mecanismos cognitivos gracias al empleo de distintos medios, como son el vídeo, audio, sonido, imagen...; Todo ello, posibilita la elección de ritmos de aprendizaje y de actividades que mejor se ajusten a los estilos de aprendizaje de los alumnos".

Además, no sólo la variedad en las propuestas permiten satisfacer necesidades específicas de los estudiantes y ofrecer una enseñanza más personalizada, sino también la cantidad de actividades ofrecidas (más de 1.500 pantallas por curso). 
7.4 Presentar tareas lingüísticas relevantes para los estudiantes y estimular la motivación del estudiante presentando la lengua en un marco cultural

En el AVE encontramos lengua real en contexto real, riqueza de documentos sonoros y escritos (textos y audios diferentes con ideas y puntos de vista diferentes) información cultural, materiales auténticos y actualizados por lo que se produce una experiencia de aprendizaje "real" sin permitir en ningún momento el uso caótico de los recursos o el desaprovechamiento de los mismos. Se puede focalizar de esta manera la atención en el desarrollo de de estrategias de aprendizaje y también de autocontrol.

En el AVE, asistimos a la búsqueda constante de un aprendizaje siempre significativo y no puramente formal. Por otro lado, se aprovecha positivamente también, la variedad de formatos y estímulos visuales de la red.

\subsection{Ofrecer un diseño que implique al estudiante y que le dé seguridad.}

Navegación intuitiva, instrucciones claras, tono adecuado, calidad en la gráfica y el formato... Los objetivos que se persiguen en cada ocasión son siempre muy claros, así como las instrucciones.

Además, como ya hemos visto, el alumno siempre puede acceder a los diferentes tipos de ayuda, y si fuese necesario, cuenta también con el acceso a un glosario. La idea es que el alumno se sienta libre y, a la vez, "amparado" y pueda contar con algún tipo de "anclaje afectivo", sea cual sea su modalidad, tenga tutor o no lo tenga.

7.6 Fomentar la construcción del conocimiento con la integración de los conocimientos adquiridos con los que ya posee y su propia experiencia

Planteamiento de situaciones que inducen a pensar y a comparar con la propia realidad y experiencia personal. De esta manera, la experiencia particular de cada estudiante cobra relevancia (especialmente en la realización de las tareas finales).

Según los principios de la teoría constructivista, las actividades del AVE se basan en la activación de los conocimientos previos del alumno y dan respuesta a los distintos estilos de aprendizaje.

\subsection{Fomentar la utilización de herramientas de aprendizaje}

Como es el caso del foro, la chat y el correo. Supone, por lo tanto, también el desarrollo por parte del alumno de la capacidad de usar las TIC para su formación, el desarrollo de su sentido crítico y de su sentido de la responsabilidad. 
Bien es verdad que no hemos analizado aquí todos los elementos que promueven la autonomía (que serían motivo de una larga publicación) sino los que, en este momento, eran de mayor relevancia y pertinencia.

\section{El AVE y las instituciones}

El AVE se revela de inestimable ayuda tanto para la organización de cualquier tipo de curso de lengua española como para la preparación, tanto en el aula, con o sin ayuda de tutor, como en total autonomía por parte del alumno de los exámenes DELE (Diploma de Español como Lengua Extranjera: http://diplomas.cervantes.es/index.jsp) o de cualquier prueba dirigida a la certificación de un nivel de lengua española siguiendo los niveles marcados por el MCER.

En el siguiente cuadro, podemos observar las diferentes correspondencias entre los cursos del AVE, los diplomas del DELE y el MCER.

\begin{tabular}{|c|c|c|c|}
\hline $\begin{array}{l}\text { Marco Común } \\
\text { Europeo de } \\
\text { Referencia }\end{array}$ & $\begin{array}{l}\text { Asociación Europea } \\
\text { di Expertos de } \\
\text { "Testing” Lingüístico } \\
\text { (A.L.T.E.) }\end{array}$ & $\begin{array}{l}\text { Diploma de } \\
\text { Español como } \\
\text { Lengua Extranjera } \\
\text { (D.E.L.E.) }\end{array}$ & $\begin{array}{l}\text { Plan Curricular } \\
\text { Instituto } \\
\text { Cervantes } \\
\text { (A.V.E) } \\
\end{array}$ \\
\hline $\mathrm{C} 2$ & 5 (Good User) & $\begin{array}{l}\text { Diploma Superior } \\
\text { de Español }\end{array}$ & - \\
\hline $\mathrm{C} 1$ & 4 (Competent User) & - & Nivel Superior \\
\hline B2 & 3 (Independent User) & $\begin{array}{l}\text { Diploma Básico de } \\
\text { Español }\end{array}$ & Nivel Avanzado \\
\hline B1 & 2 (Threshold User) & $\begin{array}{l}\text { Certificado Inicial } \\
\text { de Español }\end{array}$ & Nivel Intermedio \\
\hline A 2 & 1 (Waystage User) & - & Nivel Inicial \\
\hline
\end{tabular}

Con el objetivo de difundir el español y de ofrecer un producto a la vanguardia en calidad técnica y contenidos, el Instituto Cervantes propone el AVE a las universidades y a los centros de enseñanza de cualquier nivel interesados en la integración de las TIC en sus propias aulas.

Son muchos los centros del Instituto Cervantes que ofertan el AVE tanto en sus propios programas, como para particulares y, con condiciones siempre favorables a instituciones, en especial, centros de enseñanza reglada del español a cualquier nivel (enseñanza primaria, secundaria y, sobre todo, universitaria) o no reglada (centros privados de idiomas, etc.).

En el caso concreto del I. Cervantes de Nápoles, contamos con una oferta flexible para las instituciones y centros de enseñanza que abarca desde la organización completa de cursos (licencias y seguimiento por parte del Cervantes) o licencias para los niveles que interesen a cada centro (oferta que incluye la formación de los futuros tutores de la ins- 
titución), a oferta de licencias AULA (licencias que dan acceso a todos los cursos de todos los niveles), ideales para ofrecer material de apoyo a los estudiantes en los centros lingüísticos o laboratorios de idiomas de los diferentes ateneos. Y entre las propuestas aquí expuestas, se hallarían todas las posibles variantes que respondiesen a las necesidades concretas de los centros, necesidades que siempre estamos dispuestos a satisfacer. ${ }^{7}$

\section{Conclusiones}

En un momento en el que el aprendizaje en Internet ofrece completa confianza, el Instituto Cervantes se ha planteado y aceptado el complejo reto de llevar a cabo una auténtica y plena integración de las TIC en la enseñanza del español.

Las dificultades no son pocas, puesto que es muy complejo definir un modelo válido y generalizado y, sin duda, llegar a una normalización no será fácil, pero lo cierto es que nos hallamos en uno de los ámbitos con mayor margen para la evolución y la investigación.

Estamos ante un modelo de integración didáctica de las TIC en el aprendizaje del español basado en un recurso concreto: el AVE en el que objetivos y contenidos del currículo se reparten en función de las necesidades y disponibilidad de los destinatarios, permitiendo dar respuesta a la demanda creciente de alumnos.

Ya se opte por un itinerario cerrado, marcado por el propio sistema, con o sin tutor, ya por un recorrido más libre, se trata de un dispositivo informático que ayuda al estudiante a adquirir una mayor autonomía, respetando ritmos, estilos y estrategias de aprendizaje. Se busca que tanto profesores como alumnos vean facilitado su trabajo y que este sea más atractivo, motivador, eficaz y personalizado.

Hemos hablado de cómo gracias a una serie de factores y elementos los estudiantes "aprenden" a ser cada vez más autónomos y conscientes de su propio aprendizaje y no querría acabar sin decir que también se fomentan estos principios en los propios tutores ${ }^{8}$, entre los que yo me cuento. Estos, a través del contacto e intercambio de opiniones con otros iguales (y las herramientas para ello las proporciona el mismo $\mathrm{AVE}^{9}$ ) y a la reflexión a la que les lleva la utilización de las herramientas, materiales y espacios a su disposición, así como el curso de formación en línea, diseñado específicamente para ellos (de acceso libre) y ubicado también en el AVE, pueden convertirse, a su vez, en aprendientes autónomos.

Por último, sólo añadir que el AVE es también un proyecto de investigación en marcha, avalado por no sólo por el trabajo de un equipo de profesionales sino también por las experiencias concretas con alumnos en diferentes contextos, y caracterizado por un gran dinamismo orientado 
a la búsqueda de soluciones, respuestas para un número cada vez mayor de usuarios y mejoras, además de un deseo de ir con los tiempos. Todo ello se traduce en las revisiones y actualizaciones a las que es sometido constantemente.

En concreto en este momento y sin descuidar un ápice lo ya existente, los esfuerzos se están plasmando, entre otros proyectos, en una nueva plataforma, ya operativa con parte de los cursos, conocida entre los usuarios como AVE 2 y en la creación de un curso on-line para niños.

\section{Bibliografía básica y Webliografía}

Abad Castello, M. "Las herramientas de comunicación en el AVE. Las herramientas de comunicación de Internet en un entorno virtual de aprendizaje-enseñanza utilizado como aprendizaje autónomo", Biblioteca Virtual REDELE (Ministerio de Educación y Ciencia), número 7, Primer semestre. <http://www.mec.es/redele/Biblioteca2007/MalenaAbad.sht$\mathrm{ml}>[09 / 2007]$

Adell, J.et al. 2004. "Selección de un entorno virtual de enseñanza/aprendizaje de código abierto para la Universidad Jaime I" (Informe de la Universidad Jaume I) <http://cent.ui.es/dod/eveauji_es.pdf> [09/2007]

De Basterrechea, JP. 2005. El Español en el Mundo: Anuario del Instituto Cervantes 2005, Centro Virtual Cervantes.

$<$ http://cvc.cervantes.es/obref/anuario/anuario_05/basterrechea> [09/2007]

De Basterrechea, J.P. 2004. "Integración de los recursos didácticos digitales en la enseñanza del español. Hacia un nuevo paradigm”a, en: Identidad lingüística y globalización, III Congreso Internacional de la Lengua Española (CILE), 2004. Rosario, Argentina. Real Academia Española e Instituto Cervantes. <http://cvc.cervantes.es/obref/congresos/rosario/ponencias/ internacional/basterrechea_j.htm> [09/2007]

De Basterrechea, JP. y Juan Lázaro, O. 2006. "La integración de la NTIC en la actividad docente: el Aula virtual de españo"l. Cuadernos de Tiempo Libre, Colección Expolingua.

De Basterrechea, J.P. y Juan Lázaro, O. 2004. "Las TIC en la enseñanza de idiomas: El Aula Virtual de Español”. En: CIVE 2004, IV Congreso Internacional Virtual de Educación. Formato CD-ROM. Universidad de las Islas Baleares y Cibereduca.com

Departamento de Tecnología y Proyectos Lingüísticos del Instituto Cervantes. 2005. "El Aula Virtual de Español (AVE): un modelo de integración de las TIC en la enseñanza de E/LE”.En: m-ICTE, 2005 ( 3rd International Conference on Multimedia and Information and Communication Technologies in Education. <http://www.formatex.org/micte2005/148. pdf $>$ [09/2007]

Instituto Cervantes. 2005. "Aula Virtual de Español. Los cursos de español en Internet del Instituto Cervantes". Revista del Instituto Cervantes, n. ${ }^{\circ} 4$, 
mayo-junio de 2005. <http://www.cervantes.es/seg_nivel/institucion/ Revista_Cervantes/revista_pdf_04/rc_separata_04.pdf $>$ [09/2007]

Juan Lázaro, Olga. 2006. “¿Enseñanza de español “sin” Internet? Enciclopedia del Español en el mundo”. En: Anuario del Instituto Cervantes 2006-2007, Instituto Cervantes, Random House Mondadori y Círculo de Lectores, pp. 359-363.

Juan Lázaro, O. y De Basterrechea, JP. 2005. Influencia de los recursos digitales y los sistemas de comunicación en el modelo de enseñanza de E/LE. <http://www. mec.es/redele/biblioteca2005/fiape/juan_basterrenechea.pdf $>$ [09/2007]

En: Izquierdo, J. M.; Juan Lázaro, O.; De Basterrechea, J. P.; Alonso, M.; Prieto, R.; Mochón, A.; Lim, H.; Altmann, W. (Eds. 2005): Actas del Primer Congreso Internacional de FIAPE (Federación Internacional de Profesores de Español), Toledo, 2005. <http://www.mec.es/redele/biblioteca2005/ fiape.shtml> [09/2007]

Juan Lázaro, O. Y Olalde, S. 2001. "Cursos de español a distancia: Sistema de seguimiento y evaluación. Información para el estudiante". En: Actas del Congreso Internacional de Informática Educativa, Madrid, UNED.

Juan Lázaro, O.; Duque, A. y Gil, M. 2002. "Actividades cooperativas a través de Internet: Cursos de Español a distancia del Instituto Cervantes", En: Online Educa Barcelona, 2. ${ }^{a}$ Conferencia Internacional de la Educación $y$ de la Formación basada en las Tecnologías, Barcelona, ICWE y Universitat Oberta de Catalunya.

Hernández Mercedes, P. (2007): “El Aula Virtual de Español (AVE). Una nueva modalidad de explotación”. En: II Congreso Virtual de Enseñanza del Español como LE. 7-20 de mayo 2007. (en edición) <http://congresoele.net/> [09/2007]

Manrique Villavicencio, L. "El aprendizaje Autónomo en la educación a distancia" <http://www.ateneonline.net/datos/55_03_Manrique_Lileya. pdf $>$ [09/2007]

Mezzadri, M. I ferri del mestiere (auto)formazione dell'insegnante di lingue, Guerra Edizioni, Perugia, 2003. <http://www.ameritalia.id.usb.ve/Amerialia.002. mezzadri1.htm $>$ [09/2007]

Olalde Vegas, S.M. ${ }^{a}$ y Juan Lázaro, O. 2004. "Distance-learning Spanish courses: a follow-up and assessment system", Studies in Language Testing 18, European Language Testing in a Global Context, Cambridge University Press, pp. 221-236.

Pérez Torres I. 2006. Diseño de Webquests para la Enseñanza/Aprendizaje del Inglés como Lengua Extranjera: Aplicaciones en la Adquisición de Vocabulario y la Destreza Lectora, Granada, Editorial Universidad de Granada. <http:// adrastea.ugr.es/search ${ }^{\star}$ spi $\sim S 1 />$ [09/2007]

(Búsqueda: Pérez Torres, María Isabel)

Ruiz de Zarobe, Y. 1997. Aprendizaje autónomo en la enseñanza de segundas lengua: una experiencia en la universidad. Didáctica 9, Madrid, Servicio de publicaciones UCM, 1997, pp.183-193.

Úriz, N. El aprendizaje cooperativo. Unidad técnica de diseño y desarrollo curricular, Pamplona, Gobierno de Navarra, 1999. 


\section{Notas}

${ }^{1}$ Conocido por las siglas EVA o EVE-A, dependiendo de los autores, aunque la más frecuente es la primera.

${ }^{2}$ Entorno de administración, del tutor, del alumno, salas de comunicación y de trabajo autónomo, sistema de seguimiento y evaluación, materiales didácticos complementarios para atender a las distintas necesidades y estilos de aprendizaje, trabajo de las estrategias de aprendizaje, etc.

${ }^{3}$ MDM: materiales didácticos multimedia.

${ }^{4}$ Fue en octubre de 2006 cuando informé a la sede central sobre esta variante que podría colmar un vacío importante en la oferta AVE, al menos, en el centro en el que trabajo. Tras ser aceptada la propuesta e incluida en nuestra oferta a lo largo del curso 2006-07, podemos concluir que efectivamente ha dado una opción de estudio a posibles alumnos (personas que, a través de los diferentes canales posibles, nos pedían información acerca de nuestra oferta de cursos on-line) que, por diferentes motivos -no alcanzarse los mínimos para formar grupos con tutor, falta de interés por la propuesta de "SIN TUTOR-REGISTRADO", etc.- se hubieran "perdido" como tales (damos fe de ello por lo sucedido en años académicos anteriores). El número de alumnos que, a lo largo del curso 2006-07 han optado por esta modalidad ha sido de 26, lo que para nosotros, constituye un buen primer paso.

${ }^{5}$ Se nos ofrece cumplida cuenta de esta modalidad, en general, y de cómo está siendo aplicada en el IC de Nápoles, en particular, en HERNANDEZ MERCEDES, Pilar (2007): «El Aula Virtual de Español (AVE). Una nueva modalidad de explotación», II Congreso Virtual de Enseñanza del Español como LE. 7-20 de mayo 2007. (en edición) [en línea] http://congresoele.net/ Si algún lector desea recibir el artículo antes de la aparición de las actas del citado congreso, no tiene más que ponerse en contacto con la autora (pilarh@cervantes.es)

${ }^{6}$ Antes de empezar a trabajar o para consultar en cualquier momento que lo desee, el alumno cuenta con una Guía a la navegación específica para su tipología de licencia.

Cuenta también con:

- Botón de navegación, en todas las pantallas, que permite que el alumno se dirija al punto de los cursos que desee o que siga la secuencia recomendada.

- Enlaces a los materiales complementarios recomendados para practicar los contenidos relacionados con la actividad de la pantalla.

- Botón para registrar la actividad de forma que quede grabada en el sistema de seguimiento.

- Botón ayuda de funcionamiento, en cada pantalla, para que el alumno siempre sepa que tiene que hacer para realizar una actividad.

- Botón de acceso a la traducción de los enunciados a la lengua que escogió al acceder al AVE (en los niveles iniciales).

${ }^{7}$ Si se desea recibir la oferta a instituciones del IC de Nápoles, basta con enviar la petición a pilarh@cervantes.es. Las ofertas restantes, es decir, la de la misma programación del centro y la ofrecida a particulares se pueden consultar en la página del centro, http//:napoles.cervantes.es

${ }^{8}$ Los tutores cuentan con un entorno propio de acceso personal y restringido donde se encuentran todas las herramientas y espacios que posibilitan y facilitan su trabajo.

- El escritorio con:

- un horario para colocar las horas de tutoría;

- una ficha de cada grupo que tutorice;

- una ficha individual con los datos de cada alumno;

- un botón de acceso a todos los materiales didácticos dela plataforma.

- un enlace al Curso de formación de tutores

el acceso a la ayuda

- Banco de recursos: materiales aportados por los propios tutores. 
- Espacios de comunicación diferenciados para dirigirse a cada alumno, al grupo o para comunicarse con otros tutores.

El tutor, obviamente, cuenta con herramientas para hacer un seguimiento del trabajo de los alumnos, para asesorarles y evaluarles en un espacio propio.

${ }^{9}$ Con respecto al colectivo de tutores, las herramientas de comunicación de que disponen en su entorno les permiten:

- ponerse en contacto con otros tutores para intercambiar experiencias y consultar dudas;

- publicar en el entorno actividades adecuadas para el AVE para que otros tutores las puedan utilizar y acceder a lo publicado por otros compañeros. 



\title{
IL TUTORATO PER L'AUTOAPPRENDIMENTO RISERVATO AI PRINCIPIANTI ASSOLUTI DI LINGUA TEDESCA. VALUTAZIONE DI UN'OFFERTA DIDATTICA AGGIUNTIVA
}

\author{
Gudrun Bukies \\ Università di Cagliari
}

\section{Introduzione}

Com'è noto, l'insegnamento in tutte le sue forme e dimensioni ha tra gli obiettivi principali anche la questione di come migliorare la didattica nella speranza di renderla più efficace, a beneficio del sapere $\mathrm{e}$ delle competenze trasmesse e certamente anche dei risultati da ottenere in un determinato periodo di tempo. L'insegnamento delle lingue straniere all'università presenta, oltre alle caratteristiche e ai problemi comuni legati alla trasmissione di conoscenze, in particolare al primo anno, una serie di fattori come per esempio l'alto numero dei gruppi di apprendenti, la composizione eterogenea, e la fluttuazione ai corsi, che possono ostacolare il processo di apprendimento e rendere la didattica difficile da programmare, in considerazione degli obiettivi da raggiungere.

Con i colleghi della sezione di tedesco dell'Università di Cagliari abbiamo riflettuto su cosa si intenda per 'buon insegnamento' e nel tentativo di fissare alcune caratteristiche delle componenti coinvolte in tale processo abbiamo elaborato il seguente modello, considerando quattro aspetti fondamentali tra cui i due protagonisti: lo studente e l'insegnante. Le considerazioni teoriche e i concetti a cui si ispira il modello sono quelle descritte da diversi autori, cfr. (Holec 1980, 1988), (Little 1991), (Nodari 1996) e (Tönshoff 1997), che si esprimono a favore di una metodologia sistematica nell'insegnamento delle lingue per sviluppare negli studenti delle competenze strategiche di apprendimento. Tali strategie sono considerate fondamentali per poter gestire sia l'elaborazione delle informazioni che il processo di apprendimento in modo attivo e autonomo.

In questo quadro abbiamo evidenziato quali sono, secondo il nostro avviso, le caratteristiche delle quattro componenti coinvolte: lo stimolo allo studio e il fatto di sentirsi accettati contribuiscono ad una migliore prestazione degli studenti; la responsabilità degli insegnanti verso il proprio lavoro significa saper coinvolgere gli studenti attivamente sia nello 
Fig. 1: caratteristiche di un buon insegnamento

\begin{tabular}{ll}
\hline studenti & ognuno di loro si sente accettato, stimolato allo studio e aiutato \\
& quando sente il bisogno; \\
sono in grado di realizzare delle prestazioni ottime. & si identificano con il tipo di insegnamento; \\
accompagnano gli studenti durante il processo di apprendimento \\
complesso e individuale e li aiutano a diventare più autonomi; \\
si sentono responsabili del proprio lavoro e non si limitano ad in- \\
segnare dei concetti con una progressione lineare. \\
programma programma è inerente alla futura qualifica professionale con i \\
fondamenti teorici e l'applicazione pratica dei concetti; \\
gli apprendenti sono in grado di studiare ed approfondire gli argo- \\
menti in modo autonomo; \\
ci sono degli approcci mirati sia per gli apprendenti forti che \\
per quelli più deboli. \\
si insegnano delle competenze nella materia e delle competenze \\
di apprendimento.
\end{tabular}

studio dei contenuti che nell'acquisizione di strategie e di strumenti di apprendimento; nel programma si tiene conto della futura professione degli apprendenti e dei loro interessi personali e attraverso il metodo si intende infine costruire un'ampia gamma di strumenti che soddisfino le esigenze dei singoli apprendenti.

\section{Linsegnamento delle lingue alla Facoltà di Lingue e Letterature Straniere} dell'Università di Cagliari

Se si paragona la situazione ideale a quella con cui ci si confronta nella didattica universitaria delle lingue ogni giorno, bisogna ammettere qualche difformità. Nel seguente prospetto ho cercato di raccogliere alcune delle sue caratteristiche.

Dai due modelli risulta chiaramente che la realtà universitaria si scontra con quella di un insegnamento 'ideale'. Va comunque detto che tali difformità sono insite nel sistema accademico. A tali difficoltà, di cui soffrono un po' tutti gli insegnamenti delle lingue straniere all'Università se ne aggiungono per il tedesco delle altre, tra cui in generale:

- i principianti assoluti raggiungono una competenza paragonabile al livello B1 del quadro di riferimento europeo, solo dopo due anni, in particolare riguardo alle abilità attive come l'abilità orale e scritta;

- un'alta percentuale degli iscritti sono principianti assoluti, (o perché solo in alcune scuole si studia il tedesco, o perché un buon numero di studenti scopre l'utilità del tedesco solo dopo aver finito la scuola); 
Fig 2a: caratteristiche dell'insegnamento delle lingue all'Università

\begin{tabular}{ll}
\hline studenti & gruppi grandi; \\
& fluttuazione nella frequenza ai corsi; \\
& alto numero di principianti assoluti; \\
& alto numero di studenti che cominciano lo studio di una delle lingue \\
& in modo orientativo (inizialmente come $2^{\text {a }}$, se i risultati non ci sono la \\
& cambiano sovente in $3^{\mathrm{a}}$ lingua); \\
& studenti iscritti a corsi di laurea diversi e frequentanti un unico cor- \\
& so di lingua; \\
& in gran parte le aspettative nei confronti dei docenti e dell'insegnamen- \\
& to si basano ancora sulle esperienze fatte nella scuola. \\
& è impossibile seguire ogni singolo studente con un approccio \\
& individuale; \\
& difficilmente si è disponibili al di fuori degli orari previsti; \\
insegnanti & la priorità di raggiungere gli obiettivi prefissati e andare avanti con \\
& gli studenti che supereranno gli esami.
\end{tabular}

* p.esempio seguire le attività di scrittura individuale degli studenti (come diari, annotazioni); fare regolarmente dei test in itinere e discuterne insieme, proporre una vasta gamma di esercizi e attività diverse per esercitare tutte le abilità comunicative.

Fig. 2b: caratteristiche dell'insegnamento delle lingue all'Università

\begin{tabular}{ll}
\hline programma & $\begin{array}{l}\text { poche ore settimanali, distribuite in due semestri; } \\
\text { suddivisione in due semestri (à } 10 \text { settimane) con relativa pausa inter- } \\
\text { media relativamente lunga; } \\
\text { obiettivi didattici ambiziosi; } \\
\text { una progressione veloce per arrivare ai livelli di competenza desiderati; } \\
\text { utilizzo per lo più di libri di testo con una progressione prefissata non } \\
\text { sempre idonea riguardo agli obiettivi di una formazione accademica; } \\
\text { è difficile usare metodi o approcci differenziati o mirati per gli stu- } \\
\text { metodo } \\
\text { denti, in generale si tende ad andare avanti con i migliori (v. i motivi } \\
\text { sopramenzionati) }\end{array}$ \\
\hline
\end{tabular}

- il metodo adoperato a scuola in genere non approfondisce le attività di studio autonomo e di riflessione metalinguistica e nemmeno quella competenza linguistica richiesta nell'ambito universitario, così che anche gli studenti con tre o cinque anni di formazione scolastica devono essere 'reimpostati' ai fini degli obiettivi accademici e di uno studio più autoresponsabile;

- dopo il primo anno, molti studenti che hanno scelto il tedesco, lo cambiano in terza lingua in considerazione delle difficoltà nel superare gli esami;

- nei centri linguistici universitari i corsi di lingua non sono sempre specifici per gli studenti delle facoltà di lingue;

- prevale tra gli studenti la convinzione che il tedesco sia una lingua molto DIFFICILE da studiare; tale atteggiamento è doppiamente controproducente: 1 . giustifica le prestazioni negative; 2 . non stimola lo studio autonomo e le iniziative individuali mirate allo studio della lingua; ${ }^{1}$ 
- sarebbe particolarmente auspicabile integrare lo studio del tedesco, oltre alla frequenza dei corsi di lettorato e delle lezioni frontali, con delle attività mirate a sviluppare negli studenti lo studio autonomo e autoresponsabile. Di tale apprendimento riflessivo e cosciente gli studenti approfitterebbero anche nelle altre materie e per gli anni seguenti.

In considerazione dei fattori citati e delle difficoltà da affrontare abbiamo accolto con particolare interesse un'offerta didattica aggiuntiva proposta in via sperimentale dalla nostra Facoltà, il tutorato per l'autoapprendimento riservato ai principianti assoluti di tedesco.

3. Il tutorato per l' autoapprendimento riservato ai principianti assoluti di lingua tedesca

\subsection{L'ipotesi di ricerca}

Gli studenti principianti assoluti di tedesco costituiscono un gruppo numeroso e nel contempo delicato, e i loro fabbisogni formativi vanno tenuti adeguatamente in considerazione nel primo anno di studio all'Università. Attraverso il tutorato per l'autoapprendimento si intende promuovere le attività di studio autonomo e assistere coloro che ne sentono il bisogno, in modo da prepararli ad affrontare con successo gli esami e poter proseguire gli anni e gli studi futuri con autoresponsabilità. Nel tutorato vengono presentate strategie di apprendimento diversificate (per imparare ad imparare) e un'ampia offerta di strumenti e materiali didattici in lingua - anche interattivi e multimediali - che hanno il vantaggio di essere stati precedentemente collaudati e introdotti secondo le loro funzioni e tipologie da un tutor.

Il tutorato non intende sostituire le altre offerte didattiche dell'Università ma si dovrebbe integrare nell'insegnamento canonico rendendo in questo modo l'apprendimento della lingua straniera più intensiva, più consapevole e anche più personalizzata. L'ipotesi da cui si è partiti prevedeva che i risultati di apprendimento degli studenti frequentanti per la prima volta il tutorato per l'autoapprendimento all'Università di Cagliari sarebbero stati positivi.

\subsection{Definizioni}

Prima di spiegare in dettaglio come si è svolto il tutorato di tedesco mettiamo a fuoco i due concetti che sono alla base dell' l'autoapprendimento.

\subsubsection{L'autoapprendimento (considerazioni generali)}

- Il termine autoapprendimento o autonomia dell'apprendimento non è legato all'apprendimento delle lingue ma è un concetto pedagogi- 
co generale; tra i principi fondamentali troviamo quello che intende iniziare l'apprendente ai metodi di studio e alle tecniche di apprendimento per renderlo progressivamente capace di imparare da solo (Bimmel e Rampillon 2000);

- anche l'insegnante ha un ruolo diverso rispetto a quello tradizionale e va visto come 'stimolatore' e consulente anziché come istruttore e controllore che persegue degli obiettivi prefissati in modo lineare (Tönshoff 1997);

- Holec (Holec 1980) inserisce il concetto di autoapprendimento tra gli obiettivi formativi generali di ogni insegnamento istituzionalizzato e definisce «apprendente autonomo ideale» colui che è in grado di gestire il processo di apprendimento prendendo ogni decisione riguardo allo studio;

- l'autonomia si riferisce pertanto a un obiettivo formativo, a un concetto didattico e metodologico, a una capacità del discente e a un processo graduale guidato in primo luogo dallo studente ma seguito, incentivato e accompagnato dall'insegnante.

\subsubsection{L'autoapprendimento o autonomia di apprendimento (in ambito universitario)}

- si riferisce all'autonomia dell'apprendente, in questo caso lo studente universitario, che ha scelto di studiare una lingua straniera e alla sua capacità di studiare in modo autonomo e autoresponsabile;

- prevede forme di apprendimento in cui l'insegnante s'impegna a coinvolgere l'apprendente nelle diverse sequenze acquisizionali conferendo una graduale e maggiore responsabilità per lo studio individuale. L'offerta di materiali didattici è ricca e varia in modo che gli studenti abbiano la possibilità di creare delle connessioni tra i concetti, i saperi e i contenuti nuovi.

Oltre al termine autonomia nell'apprendimento (in tedesco Lernerautonomie, in inglese autonomy) troviamo anche l'espressione autodeterminazione nell'apprendimento - o apprendimento autoguidato (in tedesco Selbstgesteuertes Lernen e in inglese self-instruction), che non è un sinonimo di apprendimento autonomo.

\subsubsection{L'autodeterminazione dell'apprendimento}

L'espressione autodeterminazione dell'apprendimento ${ }^{2}$ è caratterizzata soprattutto dalla scelta del materiale didattico. Il materiale didattico è stato concepito ed elaborato in modo tale da permettere allo studente di gestirlo senza l'intervento o l'aiuto dell'insegnante; ciò significa che tale materiale contiene in modo esplicito o implicito tutte le istruzioni necessarie per far decidere l'apprendente da solo su come lavorarci. ${ }^{3}$ 
L'autodeterminazione è limitata quindi ai momenti in cui lo studente decide quando usare tali materiali.

Si possono riassumere i due concetti nel modo seguente: l'autonomia dell'apprendimento rappresenta una sorta di base teorica, mentre l'autodeterminazione rappresenta una tecnica all'interno di questo stile formativo.

\subsection{Una nuova forma di autoapprendimento}

\subsubsection{Il tutorato}

Come in altri ambiti legati all'istruzione, nel tutorato si tenta di mettere ulteriormente in pratica la teorie e le spiegazioni introdotte da professori e lettori tenendo in considerazione gli obiettivi didattici generali e applicando lo stile formativo sopradescritto (cfr. 3.2.1 e 3.2.2.). Il tutorato si svolge in modo interattivo, nel laboratorio linguistico della $\mathrm{Fa}-$ coltà con diversi materiali in lingua.

La durata complessiva è stata di 60 ore, distribuite in due sessioni. La prima, di tre mesi, prevedeva 6 ore settimanali concludendosi in prossimità degli esami; la seconda iniziava in Settembre per un restante numero di 12 ore.

\subsubsection{I frequentanti}

La frequenza al tutorato era consentita a un gruppo massimo di 20 persone, ovvero al numero delle postazioni col computer. Dei $67 \mathrm{stu}-$ denti, iscritti al primo anno nei tre corsi di laurea, che avevano scelto il tedesco, oltre un terzo erano dei principianti assoluti, ovvero un numero che potenzialmente avrebbe potuto seguire il tutorato

A metà tra i due semestri (gennaio 2007) gli studenti avevano già svolto una parte delle esercitazioni con i lettori ed anche il programma delle lezioni frontali con il docente. In questa fase essi sono particolarmente interessati ad approfondire degli argomenti inerenti al lessico e alla struttura della lingua, ma molto spesso non sanno bene come trovare delle risposte alle loro domande. Non hanno ancora la familiarità necessaria per andare, durante l'orario di ricevimento, a parlarne con l'insegnante. Non si sono ancora abituati al 'sistema Università', ai docenti, ai grandi gruppi nei corsi, all'anonimato. Essendo un gruppo più piccolo, nel tutorato si sentono più protetti e nella sala multimediale entrano poco a poco più facilmente in confidenza con il tutor per parlare delle proprie esigenze.

Nella prima tranche di tutorato il numero dei frequentanti variava in media tra i 5 e i 10 studenti. È un numero basso, si può sperare che col tempo e con una maggiore pubblicità interna, il numero dei frequentanti possa aumentare. 


\subsubsection{Il tutor}

Per gli studenti, in particolare quelli del primo anno, il tutor rappresenta una figura intermedia tra il lettore madrelingua e il docente che al primo anno tiene le sue lezioni in lingua italiana. Nel caso specifico la tutor è una ex-studentessa di tedesco laureata all’Università di Cagliari, anche lei a suo tempo principiante assoluto di tedesco, con esperienze di attività tutoriale precedenti sia all'Università che altrove. Il tipo di contratto del tutor per l'autoapprendimento è un co.co.co e i requisiti li abbiamo stabiliti noi della sezione di tedesco, individuando la persona tra i migliori laureati (nel nostro caso si tratta di una persona laureata con il vecchio ordinamento). È una figura diversa dal tutor d'aula di sostegno alla didattica, che prevede la presenza in aula anche del docente responsabile (sono probabilmente i tutor più conosciuti nei diversi atenei). Al momento non è prevista la stabilizzazione, né l'inquadramento, si tratta insomma di una figura nuova ancora in fase di sperimentazione.

\subsubsection{Il programma}

Il programma del tutorato riproponeva i contenuti trattati nei primi mesi nelle ore di lettorato e dal docente durante le lezioni frontali, tenendo anche in considerazione le domande e le proposte di argomenti da parte degli studenti. Si tratta pertanto di un programma aperto. Nella seconda parte sono stati proposti inoltre dei test, delle simulazioni d'esame, dei questionari di autovalutazione con l'invito a compilare regolarmente una specie di diario in cui annotare e descrivere le proprie esperienze e il rispettivo rendimento, i progressi, la valutazione delle esercitazioni, gli obiettivi da raggiungere e i problemi emersi.

Il programma del tutorato si basa quindi sui contenuti dei corsi di lingua e tiene conto degli obiettivi didattici ma la sua struttura permette ai partecipanti anche di tornare su argomenti e capitoli pregressi e di affrontare le difficoltà individuali; il momento di offrire il tutorato, a cavallo tra $i$ due semestri, si è rivelato particolarmente utile sia riguardo allo studio e al ripasso di argomenti pregressi che riguardo alla preparazione dei contenuti futuri (attraverso lo sviluppo delle strategie di apprendimento individuali).

\subsubsection{Il metodo}

Il metodo applicato nel tutorato consiste principalmente nell'offrire delle esperienze formative per incrementare la consapevolezza riguardo all'apprendimento della lingua e di creare la fiducia individuale su due livelli: 1) nel cercare di creare nello studente fiducia nelle proprie capacità cognitive tramite il possesso di determinate abilità e 2) nell'insegnargli 
le possibilità di controllare $\mathrm{i}$ fattori interni ed esterni dell'apprendimento. Lo studente deve imparare a raggiungere delle mete e a considerarsi 'autore' delle proprie esperienze. La concezione formativa è quindi orientata alla promozione dell'autodeterminazione e del senso di responsabilità.

$\mathrm{Nel}$ percorso seguito sono riscontrabili due fasi ${ }^{4}$.

Nella prima (da febbraio fino al 15 Marzo 2007) la tutor ha cercato di dedicare la prima delle due ore all'approfondimento della grammati$\mathrm{ca}$, al ripasso delle regole, che in molti casi risultavano del tutto nuove agli studenti, facendo svolgere loro dei diversi tipi di esercizi (program$\mathrm{mi} \mathrm{CD}$ e on-line). In questa fase, il ripasso delle regole spiegate già nei corsi di lingua e lo studio della lingua con metodi e in contesti diversi risultava l'attività principale (per es. tipologie sintattiche, i verbi regolari e irregolari, i verbi separabili e non separabili, la morfologia dei verbi, la memorizzazione del lessico).

Gli studenti sentivano in particolare l'esigenza di rielaborare delle regole grammaticali con del materiale diverso e di familiarizzare con dei programmi che consentissero loro una maggiore autonomia nello studio (per esempio dei programmi on-line con la correzione immediata e la spiegazione degli errori da parte della tutor). La scoperta di siti internet in lingua, di materiali on-line o degli esercizi di fonetica, collaudati e spiegati dalla tutor, erano molto apprezzati dagli studenti. Anche la spiegazione di tecniche di apprendimento e di memorizzazione e la successiva riflessione sugli obiettivi e i risultati da raggiungere hanno contribuito ad aumentare la fiducia nelle proprie capacità di studio.

La seconda ora era dedicata all'ascolto dei cd audio per esercitare la comprensione e la fonetica (per esempio degli esercizi sui numeri in cui gli studenti dovevano ripetere le cifre e in cui dovevano riconoscere le età delle persone). Poi, degli esercizi d'intonazione e accento e molti sui suoni 'particolari' del tedesco (per esempio vocali con l'Umlaut, le combinazioni tra lettere insolite per un italiano e il suono consonantico R).

In una seconda fase, notando la loro preoccupazione in vista dell'esame, la tutor ha abbandonato gli esercizi audio per concentrarsi sul programma di grammatica anche in prospettiva della simulazione d'esame che alcuni studenti avevano richiesto. Soltanto nelle ultime tre lezioni dopo la simulazione, sono stati ripresi alcuni esercizi audio (come per esempio i dittonghi e i suoni consonantici $s$ e $z$ ).

\subsubsection{Difficoltà}

All'avvio del tutorato si sono presentati veramente pochi studenti ed è stato molto difficile all'inizio convincerli a fare alcuni esercizi a casa. Alcuni per due o tre volte non li hanno mai fatti, poi quando vedevano che si correggevano e se ne parlava in classe senza problemi, li hanno fat- 
ti. Non erano abituati a riflettere sui propri stili di apprendimento, sugli obiettivi da raggiungere e sulle difficoltà e preferivano all'inizio un insegnamento di tipo frontale.

Verso metà marzo si sono formati due gruppi distinti, uno per il martedì e un altro per il giovedì. Il venerdì i due gruppi erano presenti insieme e si affrontava un nuovo argomento. Inoltre era presente una studentessa non udente e si dovevano far fare solo a lei degli esercizi on-line in particolare degli esercizi di comprensione. Nel mentre la sua accompagnatrice prendeva appunti sugli argomenti di cui si trattava al momento.

\section{Il questionario - La valutazione del tutorato da parte dei frequentanti}

Dalla valutazione tramite questionario, distribuito per comprendere meglio l'accettabilità dell'offerta formativa aggiuntiva, emergono i punti di vista dei partecipanti :

- «non ci sentiamo rappresentati nei corsi del lettorato» (forse perché purtroppo sono presenti sempre dei falsi principianti assoluti);

- «abbiamo timore di intervenire, non per gli insegnanti ma perché ci vergogniamo»;

- «vorremmo fare più test e valutazioni per avere delle indicazioni sul nostro stato di apprendimento e per vedere come si svolgeranno gli esami»;

- «nei corsi del lettorato i gruppi sono grandi, non c'è la possibilità di ritornare su determinati argomenti, vorremmo conoscere altri strumenti e programmi, per esempio degli altri testi in lingua, dei CD o dei siti internet e degli esercizi di altri libri destinati allo studio della lingua e in lingua»;

- oltre a queste osservazioni degli studenti va detto anche che la progressione nei corsi è veloce e le ore sono poche e dilazionate nell'arco di un anno accademico.

\section{L'esame di Lingua Tedesca I: alcuni dati della sessione estiva 2007}

Nell'esame di tedesco del primo anno (sessione estiva del 24 maggio 2007) i risultati degli iscritti di tedesco sono riportati nella figura 3.

Nell'anno accademico 2006/2007 si erano iscritti complessivamente per il tedesco 67 studenti, distribuiti tra i tre corsi di Laurea della Facoltà di Lingue e Letterature Straniere nel modo seguente:

- in Lingue e Culture Europee ed Extraeuropee: 27 studenti;

- in Lingue per la Mediazione Linguistica: 6 studenti e

- in Lingue e Comunicazione: 34 studenti. 
Fig. 3: risultati dell'esame di tedesco del primo anno (sessione estiva 2007)

\begin{tabular}{lc}
\hline studenti di tedesco & $\begin{array}{c}\text { numero } \\
\text { complessivo }\end{array}$ \\
\hline studenti di tedesco iscritti al I anno (a.a. 2006/2007) & 67 \\
studenti presenti all'appello di Lingua Tedesca I, maggio 2007 & 61 \\
di cui hanno superato l'esame di Lingua Tedesca I & 36 \\
studenti iscritti al I anno presenti all'appello di maggio 2007 & 30 \\
di cui principianti assoluti & 23 \\
studenti iscritti al I anno che hanno superato l'esame di Lingua Tedesca I & 15 \\
di cui principianti assoluti & 7 \\
\hline
\end{tabular}

Tra questi 67 studenti iscritti al primo anno (anno accademico 2006/2007) solo 30 si sono presentati al primo appello di Lingua Tedesca I (quello di maggio) di cui la metà (15) l'ha superato. In totale si sono presentati 61 studenti all'appello di maggio, ciò significa che 31 studenti si sono iscritti negli anni precedenti, ma sono tuttora in debito di quest'esame. Dei 61 studenti presenti all'appello di maggio, 36 studenti l'hanno superato (di cui 15 iscritti al primo anno, e 7 considerati dei principianti assoluti iscritti al primo anno. ${ }^{5}$ )

L'indice degli studenti di tedesco che superano l'esame di Lingua Tedesca I dopo un anno di iscrizione è quindi basso (e ancora più basso è il numero dei principianti assoluti che passano tale esame dopo un anno all'università).

Se gli studenti non frequentano regolarmente il lettorato (cfr. le motivazioni al punto 3) e le lezioni frontali, essi dovrebbero compensare o integrare lo studio in un modo o nell'altro, con delle attività autonome. Vediamo ora il quadro di coloro che hanno frequentato il tutorato e $\mathrm{i}$ loro voti all'esame di Lingua Tedesca I di maggio:

Fig. 4: Studenti che hanno superato l'esame di Lingua Tedesca I a maggio 2007:

\begin{tabular}{|c|c|c|c|c|c|c|}
\hline & $\begin{array}{l}\text { numero di } \\
\text { studenti }\end{array}$ & $\begin{array}{l}\text { voto più } \\
\text { basso } \\
\text { ottenuto }\end{array}$ & $\begin{array}{l}\text { voto più alto } \\
\text { ottenuto }\end{array}$ & voto medio & \multicolumn{2}{|c|}{$\begin{array}{l}\text { frequentanti del } \\
\text { tutorato } \\
\text { con risp. voti }\end{array}$} \\
\hline $\begin{array}{l}\text { superato Lingua } \\
\text { Tedesca I }\end{array}$ & 36 & 18 & 30 & 22,7 & & 7 \\
\hline $\begin{array}{l}\text { principianti } \\
\text { assoluti al primo } \\
\text { tentativo }\end{array}$ & 7 & 18 & 28 & 22 & 2 & $\begin{array}{l}18 \\
28\end{array}$ \\
\hline $\begin{array}{l}\text { con } 1 \text { anno } \\
\text { di studio del } \\
\text { tedesco }\end{array}$ & 1 & 22 & 23 & 22 & 1 & 22 \\
\hline con 2 anni & 1 & & & 18 & & \\
\hline con 3 anni & 19 & 19 & 28 & 24 & 1 & 26 \\
\hline
\end{tabular}




\begin{tabular}{lllllll} 
con 5 anni & 4 & 20 & 27 & 22,5 & & $21 ;$ \\
con $>5$ anni & 3 & 18 & 28 & 22,3 & 2 & 22 \\
$\begin{array}{l}\text { nessuna } \\
\text { indicazione } \\
\text { riguardo alle } \\
\begin{array}{l}\text { conoscenze di } \\
\text { tedesco }\end{array}\end{array}$ & 1 & & & 24 & 1 & 24 \\
\hline
\end{tabular}

Il quadro dei voti e della frequenza al tutorato dimostra che

- i risultati migliori dell'esame di Lingua Tedesca I sono stati ottenuti dagli studenti con 3 anni di formazione scolastica;

- gli studenti principianti assoluti che si preparano tramite attività di studio autonomo possono ottenere dei risultati paragonabili a quelli di studenti con esperienze formative pregresse;

- per non precludere lo studio delle lingue all'università ai principianti assoluti, l'obiettivo formativo dell'Università dovrebbe orientarsi ed offrire delle attività di apprendimento e di studio in modo da poter raggiungere dei risultati di apprendimento positivi in un anno di corso universitario;

- il tutorato per l'autoapprendimento, come quello offerto per la prima volta in modo sperimentale durante l'anno accademico 2006/2007 mostra già dei buoni risultati tra gli studenti che lo hanno frequentato. Tale attività contribuisce in modo fondamentale alla realizzazione degli obiettivi formativi. Si è deciso di allargare la partecipazione a tutti coloro del primo anno che fossero interessati, poiché accade che gli studenti che abbiano già frequentato un lettorato senza superare l'esame, esitano di fronte alla prospettiva di doverlo frequentare di nuovo con lo stesso libro, gli stessi esercizi ecc. Il tutorato può risultare idoneo proprio per loro;

- si spera di offrirlo anche in futuro, migliorando ulteriormente determinati aspetti ed intensificando la collaborazione con le altre attività didattiche, lettorato e lezioni frontali, e di allargare l'offerta ad un numero di studenti più consistente.

\section{Conclusioni}

Dai risultati ottenuti agli esami scritti della sessione estiva possiamo affermare che l'offerta del tutorato riservato ai principianti assoluti viene accolta anche da altri studenti con esperienze pregresse nella formazione del tedesco.

Se inseriamo ora il modello 3 (cfr. fig. 5) tra il modello 1 presentato all'inizio riguardo all'insegnamento ideale e il modello 2 , e cioè l'insegnamento delle lingue straniere all'università, vediamo che il modello 
del tutorato si inserisce molto bene tra i due modelli, anzi chiude il cerchio perché è un'integrazione e perché va ad arricchire l'insegnamento della lingua straniera in un contesto universitario.

Fig. 5: caratteristiche del tutorato per l'autoapprendimento

\begin{tabular}{ll}
\hline studenti & ognuno di loro si sente accettato, stimolato allo studio e aiutato \\
& quando ne sente l'esigenza; \\
& sono in grado di realizzare delle prestazioni eccellenti. \\
il tutore & si identifica con il tipo di insegnamento; \\
& accompagna gli studenti durante il processo di apprendimento com- \\
& plesso e individuale e gli aiutano a diventare più autonomi; \\
& è disposto a impegnarsi anche al di fuori degli impegni prescritti \\
& (p.esempio con contatti permanenti via email); \\
& si sente responsabile del proprio lavoro e non solo un semplice esecu-
\end{tabular}

programma offerta intensiva (6 ore settimanali) a partire dal periodo in cui non ci sono lezioni tra i due semestri con compiti da svolgere a casa che saranno corretti;

il programma è aperto in base alle esigenze dei partecipanti;

si basa sui programmi del lettorato e delle lezioni frontali;

è ricco e inerente alla futura qualifica professionale;

gli apprendenti sono in grado di studiare ed approfondire gli argomenti in modo autonomo.

metodo ci sono degli approcci mirati sia per gli apprendenti forti che per quelli più deboli.

Il tutorato non sostituisce nessuna delle altre tipologie d'insegnamento, ma per i principianti assoluti del primo anno è un'offerta aggiuntiva. Non dimentichiamo che ci sono delle persone più o meno autonome, in particolare al primo anno, d'altra parte i corsi del lettorato vanno bene anche nella loro composizione eterogenea, né tanto meno gli obiettivi didattici della formazione accademica possono essere equiparati a quelli della scuola (infatti gli obiettivi accademici implicano che si studi da soli, si rispetti un piano di lavoro, i tempi, si superino gli esami, si prendano iniziative).

Tramite il tutorato di autoapprendimento non si tratta di aver trovato IL METODO per insegnare il tedesco in un contesto universitario e tanto meno sappiamo, e non lo sapremo mai, quali esiti avrebbero conseguito gli studenti del $1^{\circ}$ tutorato, se non l'avessero frequentato e viceversa, ossia è impossibile sapere se l'esito all'esame dei non partecipanti sarebbe stato diverso se vi avessero preso parte. Resta il fatto, però, che in base ai (pur pochi) risultati della valutazione, il tutorato, così come è stato strutturato e svolto nell'anno accademico 2006/2007 è da considerare un'offerta didattica valida ai vari fini dell'insegnamento universitario. Le numerosi variabili inerenti all'apprendimento e all'insegnamento delle lingue, come anche la preparazione e la motivazione individuale degli studenti, l'impegno da parte del tutor nello svolgere la sua attività, resteranno sicuramente tali, ma dai pochi risultati ottenuti si è potuto 
dimostrare che rispettando alcune caratteristiche, il tutorato per l'autoapprendimento del tedesco per principianti assoluti allargato agli studenti del primo anno sia un'esperienza positiva. È auspicabile che possa rientrare regolarmente tra le attività formative offerte dall'Ateneo.

\section{Riferimenti bibliografici}

Bimmel P. e Rampillon U. 2001, Lernerautonomie und Lernstrategien, Langenscheidt, Kassel, München.

Edmondson W. e House J. 2000, Einführung in die Sprachlehrforschung, Francke, Tübingen, Basel (ed. originale 1993).

Holec H. 1979, Autonomy and Foreign Language Learning, Council of Europe press, Strasbourg.

Holec H. 1988, Autonomy and self-directed learning: present fields of application, Council of Europe Press, Strasbourg.

Little D. 1991, Learner 1: Definitions, Issues and Problems, Authentik, Dublin.

Nodari, C. 1996, Autonomie und Fremdsprachenlernen, «Fremdsprache Deutsch», Autonomes Lernen, Sondernummer 1996, pp. 4-10.

Tönshoff W. 1997, Training von Lernerstrategien im Fremdsprachenunterricht unter Einsatz bewusstmachender Vermittlungsverfahren, in Rampillon U. e Zimmermann G., Strategien und Techniken beim Erwerb fremder Sprachen, Hueber, Ismaning, pp. 203-215.

Wolff D. 2003, Lernerautonomie und selbstgesteuertes fremdsprachliches Lernen: Überblick, in Bausch K.-R., Christ H. e Krumm H.-J. (a cura di), Handbuch Fremdsprachenunterricht, Francke, Tübingen, pp. 321-326 (edd. originali 1989, 1991, 199533).

Note

${ }^{1}$ Gli studenti principianti assoluti al primo anno difficilmente vanno a navigare in internet su siti in lingua perché non sanno quali siti visitare e temono di non comprendere niente in assoluto quando invece gradualmente possono già consultare dei siti, in particolare contenuti di carattere culturale, o di attualità.

${ }^{2}$ (Dickinson 1987 cit. in Wolff 2003)

${ }^{3}$ Un esempio sulla applicazione del concetto nel tutorato di tedesco: nei programmi ed esercizi di ascolto la tutor aveva messo a disposizione anche le trascrizioni di alcuni testi con successivi esercizi di lessico per coloro che non erano (ancora) in grado di seguire e comprendere i programmi solamente dall'ascolto; attraverso un diario gli studenti erano poi invitati ad annotare $\mathrm{i}$ propri progressi nella capacità di ascolto e di riflettere sia sulle proprie competenze che sulle difficoltà nell'ascoltare testi in lingua. Le istruzioni non valevano genericamente per tutti, ma ognuno sceglieva la via migliore per esercitarsi in base alle proprie capacità e per riflettere successivamente su come migliorarle.

${ }^{4}$ Queste informazioni si basano sul resoconto fornito dalla tutor, Anna Maria Ferrari, Cagliari, la quale ringrazio per la sua collaborazione e disponibilità.

${ }^{5}$ I dati sono stati raccolti tramite questionario. 



\title{
THE ROLE OF LANGUAGE AWARENESS AND LEARNER COUNSELLING IN DEVELOPING LEARNER AUTONOMY: TOWARDS A MORE FLEXIBLE APPROACH TO TEACHING AND LEARNING
}

\author{
Luisa Panichi
}

Università di Pisa

\section{Introduction}

Learner autonomy can be rightly considered the implicit aim of all teaching and instruction. As teachers we strive to provide our learners with the knowledge base and the tools so that they may succeed at a personal and professional level beyond the educational setting in which their learning takes place. In this sense, all learning and teaching is projected towards the future, be it short-term (exams or qualifications) or long-term (in our professions and life-long learning).

However, as educators in today's information society - in which the quantity, accessibility and speed of information can only increase - we are confronted with a series of challenges. On the one hand, it is practically impossible to predict what specific language expertise will be required of our students in the future and, on the other, as teachers, we are no longer the main and only source of language content and knowledge but merely one of many competing sources. And not only do recent developments in technology mean that learners have greater and readier access to their target language (language input) both during in-class and out-of-class activities than ever before but their exposure to the target language is no longer confined to a specific location or time. In this sense, it can be argued that the target language has become very much a portable commodity.

So, if, as teachers, we are neither the primary source of language input nor the only authority, what is our role, and most importantly, what is the knowledge base we should be providing our students with? In addition to this, as far as English as a global language is concerned, for example, the question is no longer only about what content and what language to teach (i.e. grammar vs. lexis, general vocabulary vs. language for specific purposes, formal vs. informal, spoken vs. written, literary text vs. chat, etc.), but which Englishes should be included in the curriculum. Indeed, recent debate in the field suggests that traditional notions of standard English are increasingly irrelevant in the multicultural context of global communication and discourse (e.g. Jenkins, 2006). 
Thus, it would appear that there is need to shift the emphasis in foreign language education away from the sole provision of language input and knowledge and towards training in a variety of language learning skills. In other words, it is becoming increasingly necessary that we provide our students with the learning management skills for learning in complex systems. It is suggested here that one of the necessary skills for this type of environment is learner autonomy, i.e. the learner's ability to make the most of the language resources at his or her disposal in order to reach his or her aim of learning the target language.

This paper will argue that the Language Awareness approach to foreign-language learning contributes to laying the groundwork for autonomy training. In particular, it will discuss autonomy training in terms of the discovery of self (my needs, my learning experiences, my knowledge base, my beliefs about learning and attitudes towards the foreign language) and the other (the foreign language and its sources, my peers, my audience, my instructor). It is argued that a greater awareness of self is particularly helpful in making the most of students' encounters with the other in language learning development. In this sense, Language Awareness will be presented as a language-learning framework in which teachers and learners jointly explore and determine learning relevance and appropriacy of course and learning content.

This paper takes a look at four examples of activities in which learners are formally guided and encouraged by their language instructor to take specific steps towards developing autonomy. The examples are taken from specific teacher-learner and peer encounters at the Language Centre (Centro Linguistico Interdipartimentale) of the University of Pisa and illustrate how autonomy training can effectively take place at all stages of the language-learning proficiency spectrum, from the elementary to the advanced.

\section{Language Awareness and Learner Counselling ${ }^{1}$}

Language awareness is described in the literature in general terms as a person's sensitivity to and conscious awareness of the nature of language and its role in human life (Donmall, 1985). It includes explicit knowledge about language, and conscious perception and sensitivity in language learning, language teaching and language use (ALA website). ${ }^{2}$ It can be understood to include self-awareness, learning awareness, linguistic awareness (awareness of language and its linguistic features), meta-linguistic awareness (the ability to talk about and refer to linguistic features of language and language use) and meta-cognitive awareness. In this paper, Language Awareness is presented more specifically as a counselling framework in which the learner gives meaning to language in 
terms of its significance to himself and his learning. Thus, for uas language educators, Language Awareness is the pedagogical framework which enables us to take into account all of the learner's languages, learning experience, beliefs and culture as they relate to the learner's identity. In the examples presented below, we will see how the learner progresses via the design of specific questions and tasks from awareness of self to an awareness of the other. In particular, the interviews from the learner counselling sessions for Idoneità exam students in Example no. 1 aim at developing an awareness of self; the tasks used at the B1-B2 level as described in Example no. 2 are designed to encourage awareness of self in terms of specific learning needs via the use of authentic texts; the Tandem learner questionnaires in Example no. 3 aim at developing an awareness of self via the other (i.e. one's peer) and Example no. 4 illustrates how awareness of source and audience contributes to the development in the language competence of the advanced learner.

\section{Learner autonomy and authenticity}

In the previous section we discussed Language Awareness as a means for developing learner autonomy. But how is this achieved? What contributes to the triggering of this process?

Van Lier (1996) discusses the mutually supportive relationship between awareness, autonomy and authenticity. He explains that awareness and autonomy are processes which feed into each other: as awareness of language and language learning develop in the learner, so does his capacity for learner autonomy. Similarly, as the learner becomes increasingly autonomous he also develops further in his awareness of language and language learning. In particular, however, according to van Lier, this two-way process is further spurred along by authenticity. In van Lier's work, authenticity is used both to refer to the language the learner is exposed to (authentic language) and to the nature of language learning which should be authentic to the extent that it is meaningful to the learner. In other words, it is the scope created by authenticity that enables the learner to develop.

It may be argued that authentic materials, i.e. materials in the target language which have not been designed for the teaching and learning of language but for communicative purposes in the language community they are an expression of, may not always be suitable for use in the foreign language classroom and should only be introduced at the higher levels (intermediate or advanced). However, it is often not the material itself that is the problem but rather how it is used. In other words, in educational contexts that rely heavily on the use of authentic materials, task and syllabus design play a fundamental role in maximising the efficacy of the materials available and guaranteeing opportunities for learning. 
In the teaching and learning environment described in the introduction to this paper, it is becoming increasingly the case that learner groups are no longer homogeneous in their knowledge make up and that the "one curriculum fits all" is no longer an appropriate model in language education. Indeed, careful pedagogic design that introduces authentic materials into the curriculum has several advantages in terms of flexibility, specificity and learner training.

First, the same material can be used in a variety of ways so that the learning needs of both the lower level students and the higher level learners can be taken into account at the same time. Secondly, authentic materials provide learners with an up-to-date snapshot of the language as it is used in a specific context. This is particularly useful for the teaching and learning of highly specialised language.

Thirdly, it is suggested that the use of authentic materials also provides greater freedom for learners to make use of the language input in ways that may not have been contemplated or provided for in the original course design or lesson plan. In this sense, authentic materials allow for unprescribed and unforeseen uses which may in fact be more relevant to the learner's real needs.

Finally, it is also argued that the use of authentic materials in language education provides learners with a more robust approach to the foreign language insofar as the learners are exposed to language which is not "pre-packaged" or "watered down" in any way. This encounter with "raw" language enables the learner to develop an awareness of language as it really occurs (i.e. native speaker errors, language variety, language play, jargon, heavy reliance on a specific communicative context, voids in background cultural knowledge, etc.) and tolerance of ambiguity and miscommunication in cross-cultural communicative events (i.e. chats, forums, blogs, etc.). The robustness of the use of authentic materials also consists in the fact that they offer the opportunity for learners to engage with real world communities rather than limiting peer interaction to the less meaningful simulations of communicative tasks (e.g. role play) which were common in the traditional classroom.

\section{Learner autonomy and agency}

Van Lier (2004) develops the notion of authenticity further and introduces the concept of an 'affordance.' An affordance is a relationship of possibilities which become available to the learner who is capable of authentic or meaningful engagement with his learning environment. In this sense, it can be argued the autonomous learner is one who effectively engages with his learning environment and makes the most of the resources available therein. In the examples that will be discussed below, 
the autonomous learner, thanks to his increased awareness of his language-learning needs and his target language in general, will be able to make the most of the language input he encounters in whatever form or via whatever channel, be it a written or oral text, his Tandem partner, a film, the teacher, the textbook, a chat occasion, etc. It is this interaction with the environment that is considered a central aspect to autonomy by van Lier and is often referred to as agency. In this sense, he argues that learners are the agents of their own educational destiny (2007). In other words, the autonomous learner is an active learner who takes responsibility for his or her learning within a specific learning context. The following four examples illustrate different ways of encouraging learner autonomy and agency in given learning environments.

\section{Examples}

\subsection{Example no. 1 Teacher-learner counselling for the compulsory Idoneità tests}

A teacher-learner counselling programme has been established at the Language Centre of the University of Pisa to assist students who repeatedly fail the compulsory Idoneità exam for English at the B1 and B2 levels. Since its inception in 2005, the programme has had a $99 \%$ success rate. The $1 \%$ of students who fail to pass the test within the expected time frame following the initial counselling session are students who opt out of the programme all together. The programme consists of an initial counselling session (approximately 20 to 30 minutes) and is followed by a series of follow-up sessions. It has been observed that students preparing for the B1 level are usually able to pass the exam within 1 to 2 months from the initial counselling session and within 1 to 3 months at the B2 level. An initial face-to-face counselling session is necessary for an effective and speedy diagnosis of the student's case. However, further contact and the follow-up sessions can be dealt with successfully via email or in Skype video calls or chats.

The initial counselling session is divided into four stages: the questionnaire, the face-to-face interview including a self-evaluation task, diagnosis and the drawing-up of a study plan. Two or three follow-up counselling sessions are then scheduled according to the learner's individual situation and needs. The student plays an active role in all phases of the counselling session and all decisions are reached consensually.

\subsubsection{The questionnaire}

The aim of the questionnaire is to collect and record personal data and information both about the learner's current situation (e.g. How often 
have you attempted the exam?) and his or her foreign language knowledge in general (e.g. What languages do you know?). There is a specific section on the learner's formal instruction of English (e.g. Where did you study English and for how long?) and on his or her informal exposure to English (e.g. Have you ever travelled to an English speaking country?). In the final section of the questionnaire learners are asked to put in writing what they perceives as their weaknesses in English. The questionnaire provides input for the teacher-learner interview which follows.

\subsubsection{The interview and self-evaluation statement}

The interview proceeds along similar lines to the questionnaires and is structured so as to include any relevant information about the learner's language background that may not have been recorded in writing by the student in the questionnaire (e.g. How much time do you spend on the Internet? What sites do you go to?). The interview looks at specific learning issues and past experiences of learning English and how they may be affecting the learner's current situation. Teacher and learner discuss, in particular, the results of the Idoneità exam and how they may or may not reflect previous learning episodes. Counsellor and interviewee also discuss the learner's attitude towards learning English in general. The interview ends with a self-evaluation statement from the learner in which the learner is encouraged to identify and discuss his strengths and weaknesses and suggest effective ways of addressing them. This is followed by comments by the teacher-counsellor.

\subsubsection{The diagnosis}

The diagnosis is a summary of relevant information from the questionnaire and interview and lists the counsellor's professional opinion about the main problem areas faced by the student.

\subsubsection{The study plan}

The first counselling session ends with the drawing up of a study plan for the student based on the information collected during the session and also specifies a specific timeframe and dates for the follow-up appointments.

\subsection{Example no 2. Task design for the use of authentic materials}

The following are a list of tasks that have been designed to encourage students to make the most of the authentic materials at their disposal. These activities were assigned to students in multimedia classrooms or 
for homework and have been used in courses at the B2 and C1 levels at the Language Centre of the University of Pisa.

\subsubsection{Task 1. A Film}

Watch a film of your choice, take notes of 10 new words or expressions, and be prepared to discuss the following in class:

Why did you choose this film? Was it hard to understand? Why? What parts were easier to understand? What new words did you list? How relevant were these words to your understanding of the film? Do you think they have a specific and local meaning or are they used in a general sense? What regional accent prevailed? Would you recommend this film to your fellow students on the course? Why?

\subsubsection{Task 2. A radio listening activity}

Together with your partner go to the BBC World Service website and listen to a programme of your choice. What was the programme about? Who was talking? Was it easy to understand? How many times did you listen to it? What parts did you find difficult to understand? Why? Did the programme have a transcript? Did you read it? Was it useful? What words did you hear repeated most frequently? What accent did the speakers have? Were some people harder to understand? Why? How does this task compare to the last radio listening task you did in class? Was it easier to understand? Why? Do you think your listening skills have improved? How? Would you recommend this programme to your fellow students?

\subsubsection{Task 3. A topic of your choice (reading)}

Use the Internet to research a topic of your choice. Use at least 3 different sources. What are your sources? What search words did you use to access the information you were looking for? How does the information compare across sources? Is the language used similar? Do you think the information you have found is reliable? Do you think the sources you have found are a useful example of your target language? Write a summary of the information you have found from all sources. What words appear most frequently? Which source did you like best? Why? What are the best search words for this topic?

\subsubsection{Task 4. Opinions}

Read 3 different opinions on a topic of your choice that appear on the BBC World Service Have your say website. Whose opinion do you agree with? What language is used to express an opinion in English? Write 
and post your own opinion. Be prepared to discuss what you have written in class and to justify your opinion.

\subsubsection{Task 5. Chat}

Chat with your friend or an online contact in English for 20 minutes. Print out your exchange and be prepared to talk about it in class. Was it easy to communicate with your chat partner? Were there any instances of miscommunication? How did you resolve them? What words or expressions did you feel you were lacking? How fast were you? Do you think your interlocutor was supportive of your conversation? How? What were the most common words you used in the exchange? Can you give examples of chat abbreviations or expressions?

\subsection{Example no. 3 Tandem course questionnaires}

Tandem language exchanges are collaborative learning set-ups in which both partners are native speakers of the language the other partner wants to learn. In the exchange, learners use their native language and practice their target language for the same amount of time. One of the advantages of Tandem learning is, of course, the opportunity to practice the target language with a native speaker. However, the exchange has significant implications for learning in particular in terms of Language Awareness and learner autonomy (e.g. Little, 2006; Panichi, 2007). Panichi (2007) writes:

In perceiving his own language as somebody else's target-language, the learner is able to view the process of learning as it is experienced by others. It is argued here that it is through this very doubling of the self, the creation of an alter ego, that the learner is able to see himself and the process he is engaged in from the outside. In seeing himself through his language learning counterpart, his peer, the learner is able to distance himself from his experience and gain a new perspective. In Tandem learning, self-awareness and awareness of one's peer as a learner can be considered mutually reinforcing processes. Indeed, as one becomes aware of one's partner, one also develops an awareness of self.

Tandem exchange courses have been on offer to students studying at the Language Centre in Pisa for several years now. At the end of the course, students are asked to answer a feedback questionnaire. The questionnaires cover the following areas: Questions about the course; Questions about the student's own learning (or perception of learning); Questions about peer as helper and peer as learner; Questions about the nature of Tandem learning; Questions about future learning. Feedback from the questionnaires is then used in the end-of-course 
learner counselling sessions. The questionnaire is reproduced in the Appendix below.

\subsection{Example no. 4 Presenting and Writing for the advanced student}

As part of the C1 and C2 level courses at the Language Centre of Pisa, two additional tasks in particular are carried out in addition to those already listed in Example no. 2. These are the presentation of a topic to the class (usually a 10 minute power-point presentation) and the writing of a specific text type (a scientific abstract, a film review, an editorial, etc.). The emphasis in these two types of activities is on using language effectively for communication with a specific audience.

\subsubsection{The Oral Presentation}

The presentation work can be carried out individually or in groups on any topic. It can be limited to the presentation of factual information or include opinions. Students are expected to gather information from a variety of sources. Students' presentations are discussed in class and fellow students are asked to comment not only on the content but also on presentation style. Was the presentation easy to understand? Was it clear? Was there too much information? What about pace? What about the visual layout? At the end of their presentation, the students are expected to submit a list of "annotated sources" in which they list their sources and express a value judgment about the information and the type and quality of the language they extracted from the sources. For Internet material, students are also requested to include a list of key search words that were used.

\subsubsection{Writing a text}

For this task students are expect to produce a specific text type. Before writing the text, students are asked to find samples in their target language of the type of text they are interested in writing. These sample texts are then discussed in class in terms of appropriacy, specific language, style, register and layout. Where applicable, students are invited to produce and compare similar text types in their own language and familiarise themselves with the formal differences between the two languages (their mother tongue and their target language). This task can be carried out individually or in small groups. The written text is handed in together with the reference texts the students have been using as models and information about access to their sources. Upon completion of the tasks, fellow students are asked to discuss how the text would be received by the target audience and whether any changes are needed to make the text more incisive. 


\section{Conclusions and cautionary remarks}

This paper has attempted to provide a framework for the development and promotion of learner autonomy within the specific context of traditional European educational settings. It is argued that recent developments in information technology and the creation of an increasingly sophisticated knowledge society demand that we question our role as educators. It has been suggested that Language Awareness and its application in terms of learner counselling provide a practical and flexible way of managing change within our teaching and learning environment by providing a framework within which learners and teachers can explore and negotiate autonomy.

The additional advantages of this approach in terms of quality in language teaching and learning are several. For teachers, for example, it may result in an opportunity for reflective practice and can be viewed as a means of ensuring that they are in tune with learner needs over time. In addition, it can be argued that, by encouraging local teachers to play a more active role in materials and course design, learner needs are actually catered for more effectively and speedily than, for example, by material designers and course-book writers who are geographically and culturally removed from the specific teaching context.

However, one needs to bear in mind that this approach is based on the professional belief that autonomy is indeed one of the fundamental aims of good educational practice. If the examples of pedagogic practice that have been described here are to be successful, they have to be endorsed by the teaching and learning culture in which they take place. In other words, I would argue that autonomy is a culturally determined value and caution needs to be exercised in applying some of the approaches described above in teaching and learning contexts which may not share the same set of pedagogic beliefs or 'learning culture' (i.e. that it is 'good' to be a pro-active and independent learner, that I can learn from my peers, that the teacher is not the only source of information and authority on language, etc.). ${ }^{3}$ Thus, for pedagogic design to be effective, it always needs to allow for differences in individual and collective learning culture.

\section{References}

Donmall, B.G. (ed.) (1985) Language Awareness: NCLE Reports an Papers, 6. London: CILT.

Fairclough, N. (ed.) (1992) Critical Language Awareness. London: Longman. Jenkins, J. (2006) Current Perspectives on Teaching World Englishes and English as a Lingua Franca. TESOL Quarterly 40 (1), 157-181. 
Little, D. (1996) Learner autonomy and learner counselling. In D. Little and H. Brammerts (eds) (1996) A Guide to language learning in Tandem via the Internet. CLCS Occasional Paper No. 46. Dublin: CLCS, Trinity College.

Mozzon-McPherson, M.. Counselling/Advising for Language Learning: Setting the Context, in Search of Identity, $<$ http://www.learnerautonomy. org/MarinaIndependence39.pdf $>(01 / 08)$.

Palfreyman, D. and Smith, R.C. (eds) (2003) Learner Autonomy across Cultures. Language Education Perspectives. Basingstoke: Palgrave MacMillan.

Panichi, L. (2007) The EFL Tandem Course Experience at the Language Centre of the University of Pisa: Practical considerations and pedagogical implications. Unpublished thesis.

Schmidt, R.W. (1993) Awareness and second language acquisition. Annual Review of Applied Linguistics XIII, 206-226.

Association for Language Awareness, <http://www.lexically.net/ala/ >.

van Lier, L. (1996) Interaction in the Language Classroom. Awareness, Autonomy and Authenticity. London: Longman.

van Lier, L. (2004) The Ecology and Semiotics of Language Learning: A socio-cultural perspective. London: Kluwer.

van Lier, L. (2007) Action-based Teaching. Autonomy and Identity. Innovation in Language Learning and Teaching 1 (1), 46-65.

\section{Notes}

${ }^{1}$ For a discussion of the increasing significance of learner counselling in foreign language education, see Mozzon-McPherson at http://www.learnerautonomy.org/ MarinaIndependence39.pdf

${ }^{2}$ In SLA, Language Awareness is referred to as 'noticing' (Schmidt, 1993).

${ }^{3}$ For an extensive overview of the relationship between learner autonomy and culture, see Palfreyman and Smith (2003). 



\title{
ORDINARY LANGUAGE METAPHORS: A PLAN FOR A CORPUS-BASED RESEARCH PROJECT
}

\author{
Scott Staton \\ Università degli Studi di Firenze
}

\section{Introduction}

Conceptual metaphor theory has come to occupy a central position incurrent thinking about metaphor (Steen 2006). The theory holds that metaphor, far from being a rhetorical trope, a figue of speech charcterized by deviant language use, is central to the way we think about the world. We typically, and usually unconsciously, conceive of one area of experience in terms of another. How the mind "maps" the features of one domain onto another is revealed by everyday expressions such as "You're wasting my time" (TIME IS MONEY) of "Gas prices are rising" (MORE IS UP). The theory also claims that conceptual metaphors are consistent, that is if you can "waste" time you can also "save" time, and if prices can "rise" they can also "fall" or "plummet." It follows, according to the theory, that an examination of ordinary language should offer insights not only into the working of the mind but also into relationships among expressions that are possibly realizations of the same underlying metaphorical conceptual metaphor. If, moreover, relationships among apparently unrelated expressions such as "The bottom fell out of the market" and "Prices skyrocketed" can be clarifying by identifying a common underlying conceptual metaphor, then the potential of the theory for language teachers and learners becomes increasingly evident.

In this paper I report on an experimental course which introduced students to conceptual metaphor theory within the framework of a teaching and learning experience in which the teacher gradually relinquished control over the input learners receive and learners ultimately took responsibility for their own learning process. Students carried out individual research projects in which, selecting from a variety of methods and language resources, they produced empirical evidence in support of or against the consistency of postulated conceptual metaphors. Results were written up in a short research paper.

The course was offered a few years ago to learners studying English in a language and intercultural studies degree program, which students ac- 
cede to following the three-year first level university degree n Florence, Italy. Students had a background in literature, but most had little if any preparation in linguistics. Most were native speakers of Italian, though other European languages were represented, including French, Albanian, Rumanian, German, and English. All had some knowledge of a third and sometimes a fourth language. Lessons were held once a week for ten weeks.

\section{Getting to know the theory}

In the first part of the course students were introduced to the main tenants and the analytical approach of conceptual metaphor theory. The basic text was, and still is, the by now classic Metaphors We Live By, by George Lakoff and Mark Johnson (1980). In this book, the authors argue persuasively that metaphor is a way of thinking, an aspect of cognitive processing, rather than a purely linguistic phenomenon. At the same time, however, it is language that allows us to investigate metaphorical thinking. Everyday language, they claim, is rich in expressions that reflect underlying conceptual metaphors. These expressions may involve striking images, like "Mom's going to have a cow when she hears about this," but they may also be so banal as to escape notice entirely. This is the case of spatial notions used to conceptualize time, such as "Come between six and seven," and directional metaphors to express moods, like "I'm feeling a bit down today."

\subsection{The role of examples}

Lakoff and Johnson underpin their argument with a wealth of examples. Indeed, examples play a key role in the theory, and the examples they give are strikingly different from the deliberately bland kernel clauses of much of the literature in linguistics. Some examples may appear rather opaque to learners, such as "She's on a slow burn." Most seem to pose only minor comprehension problems, at least in the groups I have taught. One reason is that students are simply discovering that familiar expressions, like "I'm crazy about you," or "You stole my idea," can be considered as metaphors. This discovery is in itself an important step in the direction of raising metaphor awareness and arguably a significant aid in vocabulary learning and retention (Boers 2000). Another may be that basic conceptual metaphors are often common to other European languages. Thus expressions realizing LOVE IS MADNESS or IDEAS ARE OBJECTS are very likely to have equivalents or analogous expressions in other languages the learners are familiar with. This recognition of a common conceptual foundation constitutes another important step along the path to developing metaphor awareness. 
It is important for learners to appreciate the methodology underlying the selection of examples in Metaphors We Live By. The examples, while all having a familiar sound, are presumably invented. This method of presenting invented data is efficient and intuitively convincing, but learners become quickly aware that they themselves would have difficulty producing a range of appropriate examples on their own. How could they test or replicate the theory if they are not able to produce convincing data independently? One way to address this issue is to have learners produce analogous examples in their own native language. The exercise has a twofold purpose. On the one hand, the search for similar phrases shows them how easy it is to come up with example sentences. On the other, on closer examination many of the sentences produced sound perhaps plausible but ultimately unnatural in the learners' own language, very likely the result of the often mechanical translation from the English source texts. The plausibility of the examples suggests that common conceptual metaphors underlie more than one language, while the unnaturalness reveals the cultural specificity of the linguistic realizations. Indeed, it has been suggested that Lakoff and Johnson's work can be considered not so much a study of metaphor as a study of American metaphor (Violi 1998). Finally, the exercise has the effect of calling into question the validity of evidence gained through learners' native speaker introspection, as the results of the introspection may be conditioned by source text examples.

Another characteristic of Lakoff and Johnson's presentation of examples is that sentences are given as disconnected lists. The examples illustrate a range of realizations of any given conceptual metaphor but, like the kernel clauses mentioned above, they are decontextualized. To compensate for this lack of context, learners are given ("non-literary") texts showing a high density of lexis associated with selected underlying metaphors. For example, a text reconstructing a sequence of events in time will exemplify consistently the use of spatial expressions, especially prepositions, to refer to time (TIME IS SPACE). Another suitable topic is economic trends, which is likely to include numerous expressions reflecting the underlying metaphor MORE IS UP. (Examples of texts and relative exercises are reproduced in Appendix 1.) By exposing learners to complete passages rather than isolated phrases, it is hoped that students will recognize the pervasiveness of conceptual metaphor not only in the language as a whole but also as an element of textual cohesion. Moreover, repeated encounters with semantically associated words should help learners expand their vocabulary (Schmitt and Schmitt 1995).

While the problem of decontextualization can be remedied in part through the introduction of appropriately selected texts, the limits of the appeal to native speaker introspection remain in place. These limits appear even more frustrating when the object of introspection is not L1 but L2 
or even L3. It is all fine and well if Mr Lakoff sits down at his desk and comes up with scores of examples of linguistic realizations of conceptual metaphors, but how is the learner supposed to repeat the procedure or verify the results? How can the student move beyond the relatively passive phase of studying, accepting, and reproducing and progress toward the realm of autonomous learning?

\section{Using the corpus}

The answer to these questions that has been proposed for this experimental course is the language corpus. The corpus offers several tangible advantages. Firstly, the language is naturally occurring, authentic English. Whereas the learner cannot possibly draw on the vast repertory of well-formed and appropriate expressions available to the native speaker, the corpus provide a very large, reliable database. Secondly, the corpus can be searched automatically, using the many programs now available. It is not necessary to sit and think up examples or to comb through newspaper articles looking for possible conceptual metaphors. The search for metaphors can now be carried out automatically, though it is likely that human-based and computer-based searches will produce somewhat different, if comparable, results (Stefanovitsch 2007). Thirdly, the corpus is an eye-opening supplement to the available resources for any language learner who is even vaguely curious about language. The search for metaphors in the corpus can predictably result in significant by-products in vocabulary acquisition, sensitivity to collocations, awareness of grammatical patterns, and so forth. The language corpus, in sum, can not only help the learner compensate for his or her lack of reliable native speaker intuition and at the same time open up opportunities for autonomous inquiry.

I am not aware of studies that have linked conceptual metaphor theory and corpus linguistics within the framework of L2 learning, but there has been considerable activity among corpus linguists interested in metaphor theory. One of the groundbreakers in this field is Alice Deignan. Deignan authored the volume on metaphor in the Collins Cobuild Guides to English series (1995) as well as Metaphor and Corpus Linguistics published by John Benjamins (2007). She has also published a number of papers investigating specific metaphors that can serve as a model for learners who would like to carry out similar studies. The article "A corpus study of metaphors and metonyms in English and Italian" (2004), co-author Liz Potter, is a good example.

In this study, Deignan and Potter review the main claims of conceptual metaphor theory, in particular the claim that the most central metaphors are grounded in bodily experience, and underline the potential of 
the theory for cross-linguistic investigation. They also stress that metaphor theorists, mostly cognitive linguists and psychologists, are primarily interested in finding out about the mind and are understandably less attentive to the surface detail of linguistic realizations. It follows that data collection through native speaker introspection and invention satisfies the metaphor theorist's needs. Researchers in language description, on the other hand, are very much interested in the patterns of surface level structures and will tend to prefer naturally occurring language to invented sentences. These needs are best satisfied through corpus investigation (2004, 1232). The authors proceed to describe the corpus methodology used in their investigation and explain the reason for the choice of their search items, in this study nose, mouth, eye and heart for English and naso, bocca, occhio and cuore for Italian. The paper, in short, illustrates both a theoretical underpinning and a practical procedure for the use of language corpora in testing conceptual metaphor theory.

Before students can attempt to replicate this sort of investigation they must have some familiarity with carrying out corpus searches. The approach I have taken is to have them start with Google. A series of graded tasks will quickly reveal the range of information about language that can be retrieved. For example, a search for "research" and "a research," and "researches" will furnish some interesting information about the countability of the noun research. A search for "want that" will provide evidence about the complementation of want, and perhaps also about the reliability of Google as a corpus, since that-clause complements may be found in non-native speaker writing. The main advantage of Google as a starting point is that it is very easy to use and almost certainly familiar to learners.

The next step is for students to learn to use a professionally designed corpus. The most familiar and most easily accessible is the British National Corpus. The Homepage of the BNC (http://www.natcorp.ox.ac.uk/ index.xml) offers the possibility to carry out simple searches of words or expressions. Sample searches, for example with the same items that were searched with Google, will promptly reveal the advantages and disadvantages of the Homepage search. Among the advantages, learners will note the manageable number of occurrences (50) and the fact that occurrences are all given in reader-friendly complete sentences. Disadvantages include the layout of the data, which forces the reader to invest some effort to find the target word, and the limited number of occurrences (50). All learners will appreciate the fact that access to the corpus is free.

There is, however, another interface to the British National Corpus which offers which provides a far greater range of query possibilities as well as unlimited access to the corpus. This is Mark Davies's Brigham Young University site (http://corpus.byu.edu/bnc/). Davies's site is relatively user-friendly and offers a variety of options that prove handy 
when searching for metaphors, such as searches for collocations and various lexical relations. It also allows users to run queries on sub-corpora and compare results from different registers, such as academic vs newspaper. Again, a series of graded exercises can be used to introduce students gradually into the world of corpus searches. (Sample exercises are reproduced in Appendix 2).

\section{Choosing a hypothesis to test}

At this point learners should have an idea of what the main claims of conceptual metaphor theory are, how these claims have been tested by a corpus linguist, and how they could go about doing the same on their own. They should be ready to work independently. One way to begin is simply to choose metaphors that have been identified and labelled by Lakoff and Johnson, like LOVE IS A JOURNEY or SEEING IS UNDERSTANDING, and search the BNC for evidence showing that they are pervasive and coherent in the language. Learners will be familiar with the metaphors and the examples from their reading so all they need to do is check the corpus to determine whether the published examples, and similar ones they can think of, actually occur in the BNC. The procedure has the advantage of being straightforward and manageable for less ambitious learners. A potential drawback, of course, is that this mechanical search deprives students of the opportunity to tackle the analytical and methodological problem of bridging the gap between abstract conceptual metaphors and concrete linguistic realizations. Indeed, the learning project is designed to allow students to discover conceptual and semantic relationships for themselves. It must be admitted, however, and perhaps accepted, that some learners who are uncomfortable with autonomous learning may prefer this safer route.

Another approach is for students to use metaphors from the literature but not the examples. One way to do this is to go to the Conceptual Metaphor Home Page, posted by Lakoff (http://cogsci.berkeley.edu/lakoff) . The server lists over a hundred conceptual metaphors, each illustrated by just a few examples. Some in the list are far from obvious (OBLIGATIONS ARE CONTAINERS, TIME IS A PURSUER), and this in itself is an invitation to a critical researcher. The paucity of examples is also an advantage, given our purposes, as learners will have to bridge the gap between abstract and concrete themselves. Some examples may also appear idiomatic and opaque to learners, e.g. "Can you get out of doing the dishes?" (OBLIGATIONS ARE CONTAINERS), and this could lead to frustration but also to motivation.

It is worth noting in passing that this website was last updated in 1994. This means that it is already a historical document. The material on the 
site does not reflect the intensive and extensive work that has been carried out in the field since that date (e.g. the development of blending theory or the research of corpus linguists), and this is actually an advantage for our purposes, as the Home Page is essentially a supplement to the Metaphors We Live By, published in 1980.

A third option is for students to draw inspiration from their reading and to come up with their own hypotheses. Given the wealth of input from published sources, it is unlikely that learners' hypotheses will be very original, but the lack of originality may well be more than compensated by the motivation to develop their own theses.

\section{Bridging the gap between abstract and concrete}

The budding metaphor researcher now has to generate a manageable list of words that can be searched in the corpus. One way to do this is to select words from the hypothesized conceptual metaphor itself. Thus if the metaphor is HAPPY IS UP, the researcher can search "happy" (and related forms) and "up." Stefanowitsch (2004) has shown that this procedure can be surprisingly productive, as he mounts an impressive challenge to the intuitive method used in the literature on ANGER/"anger" (especially Lakoff 1987 and Kövecses 1998). Another approach, and the one I encourage students to adopt, is to consult dictionaries and thesauri. The search for synonyms and words semantically related to, say, happiness and verticality is in itself a useful exercise for learners. Gaining familiarity with available resources is another bonus. Finally, a corpus search for "up" will not find "high," "soaring," or "in seventh heaven," all realizations of HAPPY IS UP. In short, assembling a list of semantically related words by consulting available resources is both efficient in terms of research aims and profitable for the purpose of language learning.

The resources now available are far too numerous and varied to list. Dictionaries for learners often include boxes with semantic fields and CD-ROMs with mini-thesauri and small corpora. The Macmillan English Dictionary (2007) also includes metaphor boxes inspired by Lakoff and Johnson. Some print resources are based, like thesauri, on concepts rather than words, and provide rich collections of semantically related expressions. The Internet is another, and for many learners, almost the natural source. The site http://dictionary.reference.com/ offers extracts from a selection of dictionaries and thesauri. There a few problems with thesauri, of course. One is that they are designed for native speakers and entries may include far too many unfamiliar words for learners. Another is that the traditional mode of presentation is simply listing, that is no information is given about the meanings of the words in the list nor about the relationships among those. 
Some new resources have adopted graphic organizers to address both of these problems. Visual Thesaurus (http://visualthesaurus.com/) and Visuwords (www.visuwords.com) both use graphic tools to show semantic and morphological relationships among words, while at the same time limiting the number of branches, and therefore lexical items, for each entry. The latter has two advantages over the former. The first is that it is free. The second is that is based on WordNet. WordNet (http://wordnetweb.princeton.edu/perl/webwn) is an impressive project that gives not only "horizontal" relations, that is synonyms and antonyms, but also "vertical" relations (note the metaphor!), i.e. hyponyms and superordinates. The wealth of information on WordNet may be overwhelming for learners, but it may be worth bringing the resource to their attention.

Whatever sources learners choose to use, they should come up with a manageable set of items, perhaps about ten to twelve items. Some of these will prove productive and others will lead up dead end streets.

\section{The research project}

At this point, students have a working hypothesis and a list of expressions to search. In our case the initial phase of the search was carried out in a computer lab with the teacher present. The teacher may be called upon to try to solve technical problems, assist students as they carry out searches in the BNC, or help them determine whether or not a word is used metaphorically. The same function, however, can be performed by fellow students, since the lab environment is also conducive to the creation of a collaborative atmosphere. Subsequent phases of the research project can, in fact, involve pairs or small groups studying related metaphors and domains. In our case, students were given the option of working collaboratively or autonomously. The teacher maintained an advisory role, either through correspondence or in meetings during office hours.

The presentation of results was, for this course, individual and written. There is a marked preference for oral exams in the humanities faculties in Italy. It is partly for this reason that students were required to write up their results in a paper. Although this was the first opportunity to write a research paper in English for most (for many the first opportunity to write a research paper in any language), on the whole it can be said that students rose to the challenge. Some chose metaphors from the literature, others did cross-linguistic studies, still others sought to investigate metaphors they identified on their own. For an impressive example of what students were able to produce see Manuela Magnoni, "A new conceptual metaphor: RELATIONSHIPS ARE ARTEFACTS," this volume. 


\section{Conclusion}

In conclusion, in this course students of language and intercultural studies were exposed for the first time to conceptual metaphor theory and introduced to the potential for theory testing provided by language corpora. They were also given the opportunity to design and carry out a project autonomously and to write up the results in a formal research paper. The demands, in short, were multiple. Students' performances in this experiment were predictably heterogeneous. Each of these components, conceptual metaphor, the corpus search, autonomous learning, and academic writing, encountered resistance on the part of some students. Each also proved to be eye-opening and stimulating, again at least for some. Judging from student written word and the end-of-course evaluation forms, however, the overall impression is that participants found the experience at times difficult but on the whole stimulating and innovative. 
Appendix 1

Transferring concepts from one domain to another

It is quite common for people to conceive of one domain of experience or thought in terms of another. For example, understanding is often described in terms of perception. We say "I see what you mean" or "I'm totally in the dark about that" to express our ability or inability to understand something. Typically, the vocabulary used to describe the source domain ('perception' in our example) is carried over to the target domain ('understanding'). This characteristic of human thought is potentially a powerful tool in learning and remembering new vocabulary and will serve as the point of departure for our study of lexis.

\section{TIME IS SPACE}

One of the most fundamental, and perhaps universal, of transfers from one domain to another is that from space to time. Indeed, it is quite difficult to imagine temporal relations without thinking in terms of space. Of course, time and space are not the same thing, and many spatial relations, and the language used to describe them, have no corresponding configuration in time. Similarly, the conceptualisation of time requires notions and expressions which are foreign to the way we think about space. Still, the conceptual and terminological overlap between the two domains is impressive and consistent, and at the centre of this overlap lie the prepositions.

Exercise 1. The following passages are taken from a brief history of Austria. Use the prepositions in the box below to complete the text.

at from in on to

\section{Prehistory and early Middle Ages}

The geographic nature of Austria's territory, opening up towards the Bohemian-Moravian Valley and the Hungarian plains, meant that, the $7^{\text {th }}$ century $\mathrm{BC}$, this area was regularly raided and populated by belligerent Scythians, Celts and Germanic tribes. the end of the $1^{\text {st }}$ century BC, the land south of the Danube was occupied by the Romans, who the middle of the $1^{\text {st }}$ century AD, during the reign of the Emperor Claudius, founded the Province of Noricum here, with its main centres in Carnuntum (near Hainburg) and Vindobona (Vienna). 
The influence of the dominant Roman culture and civilisation over the entire region began to wane the $2^{\text {nd }}$ century $\mathrm{AD}$, during a period of increased German raids. AD 180, Emperor Marcus Aurelius died in Vindobona, in the war against the Marcomanni and Quadi tribes.

the $4^{\text {th }}$ century onwards, during the Great Migration of $\mathrm{Na}$ tions, the territories of present-day Austria saw successive waves of invading Huns, Goths and Avars. Later arrivals included Slav and Bavarian settlers. The Bavarian tribal state, established and consolidated during the $7^{\text {th }}$ and $8^{\text {th }}$ centuries, was crushed 787 when Charlemagne deposed his vassal Tassil III, the last Prince of Bavaria, and annexed his territories.

\section{The Hapsburg rise to power}

Rudolf I and his successors pursued a very successful policy of acquiring new territories. During the $14^{\text {th }}$ century, in addition to Austria, Styria, Carinthia and Carniola, the Hapsburgs gained control of Tyrol (1363) and Trieste (1382). An important contribution to the strengthening of the dynasty was made by Rudolf IV, called the Founder (1358-65), who founded Vienna University and laid the foundation stone for St Stephen's Cathedral, the church that this day remains one of the symbols of the Austrian capital. Rudolf signed a treaty with the Emperor Charles IV - Bohemian king of the Luxemburg dynasty - stating that in the event of one of the dynasties (Hapsburgs or Luxemburgs) dying out, the other would reign over both territories. This situation arose 1438 , when, following the death of Emperor Sigismund of Luxemburg, both the imperial crown of Germany and the throne of Hungary passed to the Austrian Duke Albrecht II of Hapsburg, and his death to his cousin Frederick III (1440-93), who was regarded as the last emperor of the Middle Ages.

Eyewitness Travel Guides: Austria; 2003)

(From DK 
Exercise 2. The left-hand column of the table below gives some examples of the spatial use of certain prepositions. Write down some phrases in the column to the right illustrating temporal uses.

\begin{tabular}{ll}
\hline SPATIAL TEMPORAL \\
\hline ON
\end{tabular}

ON

the pavement

the way

\section{BEFORE}

me in line / the queue

a crowd of 100,000 spectators

OVER

the hill

the city

the river

IN

the street

the box

THROUGHOUT

the house

BY

the window

WITHIN

fours walls

50 miles of Paris

Exercise 3. Prepositions are notorious for having multiple meanings. Look at the suggested answers to the previous exercise (p. 6). Are there any meanings that you had not thought of? If so, add a few expressions to your list illustrating these meanings.

\section{MORE IS UP}

Let's now turn to a different lexical area. Have you ever considered why one number is said to be 'higher' than another? Or why we speak about 'falling' prices and 'high' probability? There is a widespread tendency to conceive of 'more' and 'less' in terms of 'up' and 'down'. This tendency appears to be common to most cultures of the world and may derive 
from the simple operation of piling objects on top of one another. One significant consequence of this is that many languages use concepts and vocabulary connected with verticality to describe quantity.

Exercise 4. In the box below you will find terms that are commonly used to talk about markets, demographic trends and the like. Place them in one or more of the following categories of natural world objects and note for each the word class (e.g., noun, verb).

$\begin{array}{lllll}\text { soar } & \text { peak } & \text { bottom } & \text { steep } & \text { incline } \\ \text { dive } & \text { skyrocket } & \text { deep } & \text { slope } & \text { crest } \\ \text { plunge } & \text { top } & \text { crash } & \text { surface } & \text { plummet } \\ \text { high } & \text { rise } & \text { sink } & \text { drop } & \text { low }\end{array}$

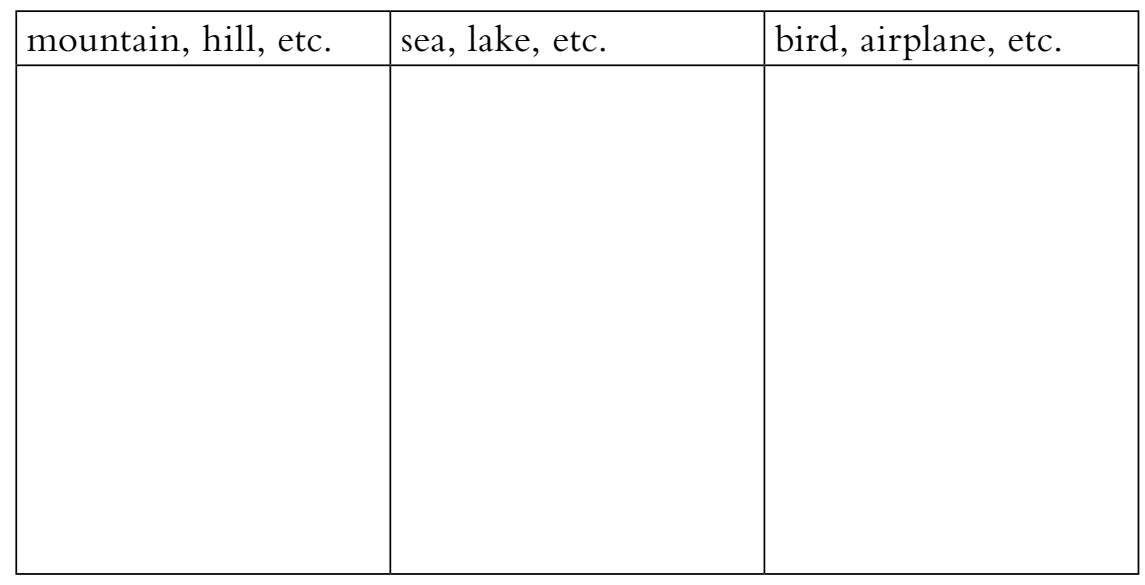

Exercise 5. Choose five words from the lists above, two you feel you can use confidently and three you feel less sure about. Write a sentence using each.

\section{Confident}

1.

2 . 


\section{Less confident}

1.

2.

3.

Exercise 6. Use the words and expressions in the box below to complete the following text. Note that some word forms may need to be changed to suit the context (e.g. 'steeper' or 'slumps').

\begin{tabular}{|llll|}
\hline prepositions & adjectives & verbs & nouns \\
over & high & sink & bottom \\
under & low & plummet & downturn \\
& steep & & slump \\
& & & decline \\
& & & ups and downs \\
\hline
\end{tabular}

Trouble in Coffee Lands

Coffee is not a trivial matter in the developing world. Coffee is the second-largest export earner for developing countries, and is the main source of foreign exchange for several nations, accounting for half of export earnings in countries like Burundi and Uganda. However, coffee prices have hitting a historic of 80 cents a kilo last October. This directly affects approximately 20 million families who live industry in the world's coffee-growing belt-between the tropics of Cancer and Capricorn - and depend on the beans for their main source of income. In March, the United Nations' World Food Program began an emergency operation to assist 155,000 people in Guatemala where a severe drought that killed subsistence crops coincided with the coffee prices. 
But for the most part, the circumstances affecting producers have gone largely unnoticed in the United States, the world's largest coffee consumer. Americans have not seen equally price for coffee in their supermarket aisles. In fact, changes in supermarket prices have been obscured by the much more dramatic expansion in the variety and sophistication of the coffee menu available to ordinary consumers. Going for $\$ 2$ a cup in many of its gourmet incarnations, coffee has become an "affordable luxury."

The stark contrast between developments in consuming and producing countries is helping to bolster the arguments for "Fair Trade" coffee: a movement that guarantees small producers a fixed minimum price for their product-between two and three times the unsubsidized market price in the current price By buying Fair Trade coffee, consumers in developed countries can feel that they make a positive difference by ensuring good working conditions and prices for farmers in poorer countries. And the firms that engage in Fair Trade also benefit by gaining a public relations advantage. But, though this clearly improves the lot of a small share of farmers, long-term options to protect a larger share of developing-country producers from prices are far more complicated.

(Adapted from Miriam Wasserman, Federal Reserve Bank of Boston; 2002)

Exercise 7. Consider the following expressions. Can you determine what underlying notion they have in common?

Under no circumstances are you to He was arrested for driving under leave the house. the influence. She's on top of her subject.

Don't worry everything is under It took days to put down the control. uprising

Even top-level managers can lose

their jobs from one day to the next. I've got you under my spell.

Ah New York, New York, big city of dreams

And everything in New York ain't always what it seems

You might get fooled if you come from out of town

But I'm down by law and I know my way around, too much

Ah too many people, too much -- a ha hah

Too much, too many people, too much, rrrrrrrah!

(Grandmaster Flash and the Furious Five, "New York, New York") 


\section{SUGGESTED ANSWERS}

Ex. 1. The original text has the following prepositions: from, at, in, in, in, from, in, to, in, on.

Ex. 2. Some possibilities are as follows.

ON: Tuesday; the eve of the battle; their arrival; arriving

BEFORE: Tuesday; getting on the train; you get on the train; long

OVER: the centuries; Easter break

IN: June; two years; time (to catch the bus); a day

THROUGHOUT: the war; the Middles Ages

BY: next Friday

WITHIN: the space of a year; the last 20 years; an hour of our arrival

Ex. 3 Different answers are possible.

Ex. 4.

\begin{tabular}{lll}
\hline mountain, hill, etc. & sea, lake, etc. & bird, airplane, etc. \\
\hline & & \\
peak $(n, v)$ & bottom $(n)$ & soar $(v)$ \\
bottom $(n)$ & dive $(v, n)$ & dive $(v, n)$ \\
steep (adj) & deep (adj, n) & skyrocket $(n, v)$ \\
incline (n,v) & plunge (v, n) & crest (n, v) \\
deep (adj, n) & surface (n, v) & plunge $(v, n)$ \\
slope (n,v) & sink (v) & plummet $(v)$ \\
crest (n,v) & low (adj, n) & high (adj, n) \\
top (n,v) & & rise (v, n) \\
high (adj, n) & & drop (v, n) \\
low (adj, n) & & low (adj, n) \\
\hline
\end{tabular}

Ex. 5. Different answers are possible.

Ex. 6. The original text has the following words: over - plummeted bottom - under - downturn - ups and downs - low - steep - declines - over - slump - higher - sinking.

Ex. 7 CONTROL IS UP 
Appendix 2

\section{LAB EXERCISE}

1. You are aware of the importance for the grammar of English, as well of other languages, of the distinction between countable and uncountable nouns. Occasionally you have doubts about how to treat a given noun, such as advice, information, or nature. You have been taught that the noun research is uncountable in English, whereas its equivalent may be used as a countable noun in many other languages, including Italian. Go to Google (www.google.com) and search the singular and plural forms of research. The simplest way to do this is to type in "research", "a research", and "researches". The google search will give you pages of links to texts with the word you are looking for. It is not necessary to go to the links. Rather, use the headings as a corpus to investigate how the lexeme research behaves in terms of countablility. In other words, you are looking for data to analyze. You are not looking for someone else's explanation of the problem.

Do the same for other nouns you may have doubts about, such as work, computer, water.

2. You know that in many languages the verb corresponding to the English want can be followed by a complement clause. For example, in Italian you can say "Voglio che tu arrivi puntuale" and German has sentences of the type "Ich will daß du pünktlich bist". You have learned that this structure is not acceptable in English, i.e. that want can be followed by a Noun Phrase object (as in "She wants the latest Springsteen album" or by an infinitival clause ("She wants to buy the latest Springsteen CD") but not by a that-clause ( "She wants that Frank buy her the latest Springsteen CD”). And still, you have doubts. Go to Google and type the following words, including the quotation marks, in the search window: "want that" and then "wants that". What is the grammatical function of "that?"

3. Now go to the website http://corpus.byu.edu/bnc /and carry out the same search,. Type the words "research", etc) without quotation marks in the $\mathrm{WORD}(\mathrm{S})$ window in the left-hand column and click on SEARCH. Compare the results of the two searches.

4. Read through the "Overview: Introduction" section of the homepage. Click on the boldface phrase "registers". One example given is that of "good + [noun] in fiction". Click on the sample search. After examining the results, go to the left-hand column of the homepage and find Register. Select the register Academic and click on SEARCH at the bot- 
tom of the column. What provisional observations can you make about the use of good in these two registers?

5. Again in the "Overview: Introduction" click on "search for surrounding words" and try out a couple of the sample searches. When you feel comfortable with how the command works, substitute the verb "look into" in the sample search with "attack" and click on SEARCH. Examine the results for illustrations of the ARGUMENT IS WAR metaphor. Try the same search selecting the sub-corpus "Academic." What can you "attack"? An opinion, a position, an argument, an idea, a word? What other forms of the lexeme attack would you need to search to e exhaustive? Try the same search with "instigate" or another verb you are interested in.

Now try the sample search with smile. Once you have examined the adjectives that combine with "smile" substitute this noun for another that may interest you. For example, you are curious to see whether the word influence is conceptualized on the basis of the MORE IS UP metaphor. One way to test this is to see whether the adjectives that combine with influence are compatible with the notion of a vertical axis. For example, can we say "high influence" or "falling influence"? Does the corpus list adjectives that are compatible with this notion?

\section{References}

Boers, Frank. "Metaphor awareness and vocabulary retention." Applied Linguistics 21 (2000): 553-571.

British National Corpus < http://www.natcorp.ox.ac.uk/index.xml $>(03 / 09)$.

Conceptual Metaphor Homepage $<$ http://cogsci.berkeley.edu/lakoff $>(03 / 09)$.

Davies, Mark. (2004-) BYU-BNC: The British National Corpus. Available online at <http://corpus.byu.edu/bnc >(03/09).

Deignan, Alice. Collins Cobuild Guides to English 7: Metaphor. London: HarperCollins, 1995.

Deignan, Alice. Metaphor and Corpus Linguistics. Amsterdam: John Benjamins, 2005.

Deignan, Alice and Liz Potter. "A Corpus Study of Metaphors and Metonyms in English and Italian." Journal of Pragmatics 36 (2004): 1231-1252.

Dictionary.com $<$ http://dictionary.reference.com/ $>$.

Google www.google.com(03/09).

Kövecses, Zoltan. "Are there any emotion-specific metaphors?" In: Angeliki Athanasiadou and Elzbita Tabaskowska (eds.), Speaking of Emotions. Conceptualization and Expression, 127-151. Berlin and New York: Mouton de Gruyter, 1998.

Lakoff, George. Women, Fire and Dangerous Things: What Categories Reveal about the Mind. Chicago, Chicago University Press, 1987. 
Lakoff, George and Mark Johnson. Metaphors We Live By. 1980 Chicago: Chicago University Press, 2003.

Steen, Gerard. "Metaphor: Stylistic approaches." In Keith Brown (ed.), The Encyclopedia of Language and Linguistics, $2^{\text {nd }}$ ed., Vol. 8, 51-58. Oxford: Elsevier. 2006.

Stefanowitsch, Anatol. "Words and Their Metaphors: A Corpus-Based Approach." In Anatol Stefanowitsch and Stefan Th. Gries (eds.) Corpus-Based Approaches to Metaphor and Metonymy. Trends in Linguistics 171. Berlin and New York, Mouton Gruyer, 2006. 61-105.

Viola, Patrizia. Introduzione all'edizione italiana. Metafora e vita quotidiana. By George Lakoff and Mark Johnson. Trans. Patrizia Violi. 1998. Milano: Strumenti Bompiani, 2007. 11-14.

Visualthesaurus $<$ http://visualthesaurus.com/ $>(03 / 09)$.

Visuword $<$ www.visuwords.com $>(03 / 09)$.

WordNet < http://wordnetweb.princeton.edu/perl/webwn >(09/09). 



\title{
A NEW CONCEPTUAL METAPHOR: RELATIONSHIPS ARE ARTEFACTS ${ }^{1}$
}

\author{
Manuela Magnoni \\ Università degli Studi di Firenze
}

\section{Introduction}

Lakoff and Johnson, in their 1980 study on metaphors (Lakoff and Johnson, 1980), suggest that metaphor is not just a trope, a particular and extraordinary figure of speech used in rhetoric (Steen, 2006), as most people think. Metaphor is, on the contrary, much more pervasive in our everyday life, not only in language, but also in thought and action. Metaphor in language is only the empirical result of how our conceptual system works, since "the human conceptual system is metaphorically structured and defined" (Lakoff and Johnson, 6). Indeed, according to Lakoff and Johnson's theory, we are used to thinking of a certain thing in terms of another one, creating a relationship between two semantic spheres (called "domains") in order to better understand the main concept we are talking (and so thinking) about. In order to achieve this target, the source domain - the conceptual domain from which we draw metaphorical expressions ${ }^{2}-$ is usually taken from the bodily experience world, while the target domain - the conceptual domain that we try to understand ${ }^{3}$ - is usually an abstract concept which can be classified and quantified through the conceptual metaphor. For example, the sentences "He attacked every weak point in my argument", "How do you spend your time", or "I gave you that idea", are based respectively on the conceptual metaphors: argument is war, time is money, and ideas are objects (Lakoff and Johnson, ch. 2, 3).

Lakoff and Johnson's study has become a cornerstone in the field of cognitive linguistics and a lot of researchers have followed their path. Some of them have developed their theory, some have defined new ways of analysis, others have found new conceptual metaphors. Nowadays, the conceptual metaphor theory is still developing, and its literature is very copious.

On the basis of Lakoff and Johnson's theory, and their fellow researchers, I will try to determine if the conceptual metaphor (found by personal intuition) RELATIONSHIPS ARE ARTEFACTS can be considered true. 


\section{Method of analysis}

This paper aims at demonstrating that RELATIONSHIPS ARE ARTEFACTS is a well-grounded conceptual metaphor. In order to prove this thesis, I will consider the linguistic expressions that the conceptual metaphor produces in everyday language. Since English is not my native language and even native speakers, as Alice Deignan suggests, "are not good at producing naturalistic language data when asked to do so consciously" ("A corpus linguistic perspective", Booklet, 18), I will use the web site View: Variation In English Words and phrases ${ }^{4}$ based on the onehundred million word British National Corpus. Through different kind of queries, this Web site makes it possible to search for certain words and phrases or surrounding words (verbs, nouns, prepositions, adjectives or adverbs) collocated within a ten-word window. It also enables the analysis of lexemes belonging to different kinds registers, always with the indication of the number and the percentage of frequency in the Corpus. Furthermore, it is possible to work on two different words comparing the result of the queries in a single window. Other Web sites are available, ${ }^{5}$ but View is the most approachable and at the same time the richest in the number of queries available. ${ }^{6}$

My analysis will start with the study of surrounding words (especially verbs and adjectives) of the lexemes that belong to the semantic field of "relationship", such as "relation(s)", "relationship(s)", "friendship(s)", "kinship", "marriage", "brotherhood", "alliance(s)", "love", "contact", "nearness". I will consider verbs and adjectives that fit both into the source domain (ARTEFACTS) and into the target domain (RELATIONSHIPS) and, in order to have a clearer view of the data, I will use the "comparative query" ceptual domains. Then, I will analyse the examples given by the corpus.

After analysing the empiric data, I will start to study the way in which the conceptual metaphor RELATIONSHIPS ARE ARTEFACTS can be considered "natural": I will examine the RELATIONSHIPS-AS-ARTEFACTS MAPPING analysing the "epistemic" and the "ontological" correspondences between the source and the target domain.

Finally, in order to demonstrate that RELATIONSHIPS ARE ARTEFACTS is a figure of thought rather than a figure of language, I will briefly consider the use of metaphorical expressions related to this conceptual metaphor in other languages.

\section{The conceptual metaphor RELATIONSHIPS ARE ARTEFACTS}

In the corpus, the most representative verbs and adjectives that are linked to "relationship" and its synonyms are: form, forge, make, create, 
handle, use, value, break, and fine, precious, original, wonderful, practical, authentic, exclusive. Analysing the surrounding verbs of "alliance(s)", one of the lexemes in the semantic field RELATIONSHIPS, what we can see at first glance (Table n. 2 in Appendix), ${ }^{8}$ is that most of the verbs require a concrete ENTITY ${ }^{9}$ as participant: it is possible, for example, "to form the clay into a vase", "to make a car", "to forge metals" or "to build a house". However, in this context, these verbs have an abstract concept, "alliance", as grammatical object. In the corpus it is possible to find expressions such as:

(1) ... and they benefit party political groups, their ability to form alliances and the bureaucracy." The much vaunted independence of the Länder is ... ... often spent in political and social spheres of activity to form alliances in peacetime and during war, as well as compensation for death and ...

(2) ...companies. The best companies are able more readily to make alliances or purchase technology or be welcomed into countries other than their own, ...... help who our brothers and sisters are but we can make alliances of people on issues of liberation and freedom that goes beyond that narrow ...

(3) ... and Scotland. It was in trying to forge the necessary alliances and to equip the essential forces that Edward then came into conflict with ....... is hardly surprising that, among those who wish to forge alliances with environmentalists, those who, like myself, work for the goal ...

(4) ....united-front tactics (stemming from the Soviet leadership's need to build alliances to counter threats from Germany and Japan) gave the Communist ...

While the expressions in the examples number 1 and 2 can be possible because of the polysemic meaning of the verbs form and make ${ }^{\mathbf{1 0}}$, even if the main meaning always requires a concrete "resultant object"11, the verbs forge and build in the examples number 3 and 4, require a particular kind of concrete "resultant object": an ENTITY that has to be CREATED by shaping a material or assembling and joining parts or materials. ${ }^{12}$ Analysing the verbs and the expressions that govern all these verbs - ability to, activity to, are able to, can, trying to, wish to, need to - we notice that the more general verbs form and make are taken by a verb or an expression concerning ABILITY, a "special skill or aptitude" (Webster's), that categorises them in a more specific semantic field; while the more specific verbs forge and build are accompanied by verbs that express an effort, e.g. trying to forge, wish to forge, or a necessity, e.g. need to build. Therefore, "alliance" is conceptualised as a CONCRET OBJECT that requires a specific (sometimes difficult) PROCESS OF CREATION 
and a particular ABILITY in order to make it. It can also be a result of a will or a necessity.

If we look at other types of "relationship", such as "friendship", "marriage", or "love", we can also find further verbs and adjectives related to these words that better define what kind of ENTITY the domain RELATIONSHIPS is conceptualised into. Most of the verbs and adjectives that fit with the RELATIONSHIPS domain can be divided in three major semantic fields: FRAGILITY, SOLIDITY and PRECIOUSNESS. For example, as for FRAGILITY, the RELATIONSHIPS domain is often conceptualised as an object easy to damage and to brake that has to be kept with care and attention:

(1) I feel it can only damage a wonderful friendship." Her son said: "They are just friends ... ... (2) Dr Johnson was right to say that we should keep our friendships in good repair, ... ... (3) Friendships are not particularly durable. Over time, they alter and change ... ... (4) Olwen had had her troubles as well. Her marriage had broken up, and she had gone to live with a man ... ... (5) Henry and Mary split up one year ago......

Regarding SOLIDITY, the RELATIONSHIPS domain is conceptualised as something with a strong consistency and difficult to break:

(1) And I want to thank those writers with whom solid friendships were formed...... (2) but with the strong love that unites all to seek the good of each and the happiness of ... ... (3) It would seem that you have a basically strong marriage if you have lived through, and overcome, your husband's affair ..... (4) there are few firmer friendships in cricket anywhere in the world than that between John... ...

Concerning the semantic field of PRECIOUSNESS, RELATIONSHIPS is conceptualised as something difficult to obtain that can be valued and appreciated:

(1) effect on other relationships. Such revision could increase the value of friendships, autonomy and other forms of creativity, and open up discussion ...... (2) and warmth abound in their presence --; I so appreciate their friendship, especially when my wife was ill with leukaemia." In February ... ... (3) do favours for unknown playwrights, no matter how old and valued their friendship. She wasn't about to let him see her reaction, though ... ... (4) were sent to her, and any attempt to win their friendship was met with sullen silences or rebuffs... ...

According to Lakoff, the source domain of a conceptual metaphor has to belong to a category that includes basic-level categories ("The contemporary theory"). As for the target domain RELATIONSHIPS, its 
superordinate source domain category (a sort of common denominator) could be ARTEFACTS. ${ }^{13}$ Indeed, to shape an artefact requires particular abilities; the artefact can be fragile (if it is made, for example, of crystal) or solid (a wooden artefact), it can be precious (a Venetian glass) or worthless (a kitchen utensil). Following an "a posteriori approach" the conceptual metaphor RELATIONSHIPS ARE ARTEFACTS seems to work. Therefore, in daily life, we can experience relationships in terms of artefacts. Then, through a set of correspondences, the metaphor would be understood as a mapping from the source domain (ARTEFACTS) to the target domain (RELATIONSHIPS), named RELATIONSHIPSAS-ARTEFACTS MAPPING (Lakoff, "The Contemporary Theory”). According to Lakoff, it is possible to examine a conceptual metaphor analysing these correspondences that are classified into ontological correspondences and epistemic correspondences (Lakoff, "Women, Fire"). The ontological correspondences are "correspondences between the entities in the source domain and the corresponding entities in the target domain", while the epistemic correspondences are "correspondences between knowledge about the source domain and corresponding knowledge about the target domain" (386-7). Therefore we can schematise the RELATIONSHIPS-AS-ARTEFACTS MAPPING as follows: ${ }^{14}$

Source: ARTEFACTS

Target: RELATIONSHIPS

Ontological correspondences:

- The artefact is the relationship.

- The artisan is the person who creates the relationship. ${ }^{15}$

- The skill of an artisan is the attitude of a person to form a relationship.

- The components of the artefact are the components of the relationship.

- The manufacture process of the artefact is the creation process of the relationship.

- The artefact breakage is the end of the relationship.

- The difficulty of creating an artefact is the difficulty of establishing a relationship.

- The care of the artefact is the correct behaviour to maintain the relationship.

- The irreproducibility of an artefact is the uniqueness of a relationship.

Epistemic correspondences:

- Source: An artisan can produce more or less precious artefacts depending on his skill.

- Target: A person can form good or bad relationships depending on his attitude.

- Source: A greater skill of an artisan produces precious artefacts, while a lesser skill produces worthless artefacts. 
- Target: If a person is gifted for relationships, the relationship will be richer, while if $s /$ he is less able to form bonds the relationship will be poorer.

- Source: Depending on the kind of artefact, it needs different parts.

- Target: Depending on the kind of relationship different components are required.

- Source: If the parts of an artefact are well joined together, the artefact is solid, otherwise it is fragile.

- Target: If the components of a relationship are well joined together, the relationship is hard to damage, otherwise it could be easily broken.

- Source: If a component of an artefact is damaged, the artefact does not work.

- Target: If a component ${ }^{16}$ of a relationship is damaged, the relationship ends.

- Source: If an artefact is kept with care, it has less possibilities to be damaged.

- Target: If a relationship is well safeguarded, it is unlikely to end.

These are only some of the possible correspondences between the source and the target domain. Indeed, as Lakoff argues,

Mappings should not be thought of as processes, or as algorithms that mechanically take source domain inputs and produce target domain outputs. Each mapping should be seen instead as a fixed pattern of ontological correspondences across domains that may, or may not, be applied to a source domain knowledge structure or a source domain lexical item. Thus, lexical items that are conventional in the source domain are not always conventional in the target domain. Instead, each source domain lexical item may or may not make use of the static mapping pattern. If it does, it has an extended lexicalised sense in the target domain, where that sense is characterized by the mapping. If not, the source domain lexical item will not have a conventional sense in the target domain, but may still be actively mapped in the case of novel metaphor. (Lakoff, "Motivation", in "The Contemporary Theory")

Considering the artefact as a VALUABLE COMMODITY that can be bought or sold, it would seem that there were no correspondences in the target domain. As we have seen above, a relationship can be valued but, apparently, it cannot be bought or sold. Verifying in the corpus, out of one thousand entries ${ }^{17}$ I was not able to find expressions that associate the verb sell/sells/sold with some kind of relationship. However, it is possible to find some sporadic expressions in which the verb buy has a relationship as grammatical object:

- I don't care too much for money, money can't buy me love ...

- Any two behaviours that clearly have been acquired by faulty learning. 
They may include destruction of parents' belongings, stealing money to buy friendship, truanting to avoid failure at school

The first example quoted is an extract from a Beatles song, then, it belongs to a particular kind of register. The second one is an extract from a social science book about behaviour disorders in children, and the verb is used metaphorically to emphasize the negative behaviour of the children who think that friendship can be bought or sold. In the corpus it is possible to find further sporadic examples of particular lexical cross domains, such as:

Milton would refresh his contacts, burnish new friendships and influence even more people.

All these examples prove that even if some source domain lexical items do not have a conventional sense in the target domain, they can still be actively mapped (and easily understood) in the case of new linguistic metaphors. ${ }^{18}$ It is possible to produce an unlimited number of new linguistic metaphors transferring lexemes from the source domain to the target domain, and we can be sure that the addressee will understand easily the message. ${ }^{19}$ Therefore, the conceptual metaphor RELATIONSHIPS ARE ARTEFACTS seems to work.

Since conceptual metaphors are not a matter of language but a matter of thought, most of them are common in different languages, above all in the same cultural area. This is the reason why the use of conceptual metaphors is theorised by some linguistics (Boers, Deignan, Gabry囚, Solska), as a way to approach the teaching of L2. They suggest that the awareness-raising through comparison of (linguistic) metaphors in L1 and L2 is a useful approach to helping learners to understand and appropriately produce metaphors, simplifying the acquisition of vocabulary and facilitating learning grammar (Deignan, Gabry区 and Solska; 352-3) .

In order to be a true conceptual metaphor, RELATIONSHIPS ARE ARTEFACTS has to produce lexical metaphors not only in English, but also in other languages. In Italian and Spanish, we easily find some expressions generated by the conceptual metaphor RELATIONSHIPS ARE ARTEFACTS: one of them is the Italian expression "stringere un'amicizia" and its Spanish parallel "trabar amistad" (literally "to tighten a friendship"). In these expressions there is an interaction between metaphor and metonymy, precisely it is a synecdoche within a metaphor. Indeed, the whole expression is understandable if in our mind friendship is already conceptualised as an artefact composed by different parts (a cross-domain mapping, from the source to the target domain ). However, "stringere un'amicizia" and "trabar amistad" 
stand for "tighten the parts that compose the artefact which friendship is conceptualised in". In this expression the parts stand for the whole, so "stingere un'amicizia" is a synecdoche, an intra-domain mapping in the semantic source domain ARTEFACTS. Unfortunately, as regards American Sign Language (ASL),${ }^{20}$ I have found only two simple "dictionaries" (ASL for beginners; Learning Sign Language) that list only words and not phrases; as a result, it is impossible to carry out studies in metaphor or metonymy. However, considering Table n.3 in the Appendix, which shows words connected to different kinds of relationships, it is possible to notice a "conceptual division" between two different groups: the words husband, wife, friend, sweetheart and the words aunt, brother, father, mother. We can call the first group of words "agentive subjects" since one is (more or less) active in creating love and friendship relationships. On the other hand, we can call the second group of words "affected subjects", ${ }^{21}$ since one does not have an active role in creating family relationships: one has a mother, a father, a brother, but s/he has not chosen to have them. In ASL "agentive subjects" are explained with signs of strong connection and link between two parts, while "affected subjects" are explained with other signs that do not pertain to "union". Therefore, even in ASL "conscious" relationships are conceptualised as ENTITIES composed of parts that have to be well connected in order to get the relationship work. This is one of the basic-level categories that belong to the superordinate category ARTEFACT, and it makes the conceptual metaphor RELATIONSHIPS ARE ARTEFACTS true even in ASL.

\subsection{Some clarifications}

\subsection{Difficilties in explaining conceptualisations}

Considering the ontological and epistemic correspondences in RELATIONSHIPS-AS-ARTEFACTS MAPPING, I have found difficulties in explaining the way in which the subjects involved in a relationship are conceptualised. Indeed, at first glance, it seemed to me that the same conceptual metaphor produced different lexical metaphors assigning to the subjects involved in the relationship two different semantic roles in the source domain. In the expression (1) She is able to form wonderful friendships the subject involved in the relationship (SR) is conceptualised as the "creator", the "artisan" of the artefact/relationship; in the expression (2) Susan is very close to Sam the SR is conceptualised as one of the "parts" that compose the "artefact".

Analysing these examples, I realised that (1) is produced through a metaphor process, while (2) is the result of two processes, first of metonymy and then of metaphor. It is what Goosens calls "metaphor from 
metonymy", and it is possible when "an expression develops a meaning through metonymy and that meaning is then mapped metaphorically onto another domain" (qtd. in Deignan; ch. 3.2). Indeed, this expression can be understood on two levels: literally and figurative. For example, Susan may be physically close to Sam, as a manifestation of love or tenderness; but in a figurative sense the physical closeness may be absent, and the expression will refer only to the sphere of feeling. On the other hand, in the example (1) the lexical metaphor is directly generated by the conceptual metaphor, as I have demonstrated in the paper, so it confirms the semantic role "artisan" assigned to the subject.

\subsection{Agentive vs experiencer roles}

Another difficulty arose considering the expression Sarah is in love. ${ }^{22}$ Apparently, this expression places Sarah inside the relationship as part of the artefact/relationship, and not as the creator. However, we have to distinguish the semantic role of the "agentive subject" from the semantic role of the "experiencer subject". In this case Sarah is an "experiencer subject" since she does not decide to fall in love. She is like a puppet moved by a superior entity -that can be called Faith, God, Chance- that puts her, like a piece, into the artefact that the artisan/entity assembles and shapes. Therefore, the conceptualisation of the subject involved in the relationship as "artisan" seems to work also in this case. Indeed, the "artisan" is not the grammatical subject Sarah, but the implicit subject "superior entity".

\section{Conclusions}

By analysing the corpus, I have found that most of the verbs and adjectives that refer to some kind of relationships are usually used to refer to a particular concrete entity: ARTEFACTS. Both relationships and artefacts can be precious, original, or wonderful and they can be created, forged, formed, used, valued, or broken. Through this study, considering also some problematic expressions, I have tried to prove that the RELATIONSHIPSAS-ARTEFACTS metaphor is well-grounded on both ontological and epistemic correlations.

In order to be a conceptual metaphor, RELATIONSHIPS ARE ARTEFACTS has to generate linguistic metaphors not only in English, but also in other languages. Therefore, I have briefly taken into consideration three other languages, Italian, Spanish, and ASL, and I have found that the conceptual metaphor produces expressions in them as well. In short, the conceptual metaphor RELATIONSHIPS ARE ARTEFACTS seems to work. 


\section{Appendix}

Table n. 1: A "comparative window" of surrounding verbs related to friendship and object. (View)

\begin{tabular}{llccc}
\hline FRIENDSHIP + & \# FRIENDSHIP & \# OBJECT & \% FRIENDSHIP \\
\hline 1 & RENEW & 5 & 0 & $100.0 \%$ \\
2 & FORM & 5 & 1 & $83.3 \%$ \\
3 & NEED & 5 & 5 & $50.0 \%$ \\
4 & ESTABLISH & 4 & 0 & $100.0 \%$ \\
5 & LEAD & 4 & 0 & $100.0 \%$ \\
6 & WANT & 4 & 1 & $80.0 \%$ \\
7 & WIN & 4 & 1 & $80.0 \%$ \\
8 & EXTEND & 4 & 2 & $66.7 \%$ \\
9 & CONTINUE & 4 & 2 & $66.7 \%$ \\
10 & KEEP & 4 & 3 & $57.1 \%$ \\
11 & BUILD & 4 & 4 & $50.0 \%$ \\
12 & FIND & 4 & 9 & $36.4 \%$ \\
13 & THINK & 4 & $30.8 \%$ \\
14 & SEE & 43 & $14.8 \%$ \\
15 & COUNT & 4 & 0 & $100.0 \%$ \\
16 & CONCLUDE & 3 & 0 & $100.0 \%$ \\
17 & CULTIVATE & 3 & 0 & $100.0 \%$ \\
18 & ENJOY & 3 & 1 & $75.0 \%$ \\
19 & RESULT & 3 & 1 & $75.0 \%$ \\
20 & COME & 3 & 1 & $75.0 \%$ \\
21 & OFFER & 3 & 4 & $42.9 \%$ \\
22 & GIVE & 3 & 5 & $37.5 \%$ \\
23 & PROVIDE & 3 & 9 & $25.0 \%$ \\
24 & SAY & 3 & 19 & $21.4 \%$ \\
25 & USE & 3 & & $13.6 \%$ \\
\hline
\end{tabular}


Table n. 2: Surrounding verbs of the word Alliances. (View)

\begin{tabular}{|c|c|c|c|c|}
\hline & WORD & \# TIMES NEARBY & TOTAL IN CORPUS & $\%$ NEARBY \\
\hline 1 & FORM & 11 & 34173 & $0.0 \%$ \\
\hline 2 & MAKE & 7 & 77393 & $0.0 \%$ \\
\hline 3 & FORGE & 6 & 461 & $1.3 \%$ \\
\hline 4 & CREATE & 4 & 8102 & $0.0 \%$ \\
\hline 5 & SEEK & 3 & 5357 & $0.1 \%$ \\
\hline 6 & ENTER & 3 & 5176 & $0.1 \%$ \\
\hline 7 & BUILD & 3 & 7142 & $0.0 \%$ \\
\hline 8 & DEVELOP & 3 & 8541 & $0.0 \%$ \\
\hline 9 & CONSTRUCT & 2 & 1401 & $0.1 \%$ \\
\hline 10 & BELONG & 2 & 2043 & $0.1 \%$ \\
\hline 11 & ESTABLISH & 2 & 5214 & $0.0 \%$ \\
\hline 12 & ENCOURAGE & 2 & 5071 & $0.0 \%$ \\
\hline 13 & STRIKE & 2 & 5599 & $0.0 \%$ \\
\hline 14 & RE-ASSEMBLE & 1 & 11 & $9.1 \%$ \\
\hline 15 & ABROGATE & 1 & 16 & $6.3 \%$ \\
\hline 16 & ESCHEW & 1 & 72 & $1.4 \%$ \\
\hline 17 & CIRCUMVENT & 1 & 121 & $0.8 \%$ \\
\hline 18 & MOBILIZE & 1 & 146 & $0.7 \%$ \\
\hline 19 & COUNTERACT & 1 & 268 & $0.4 \%$ \\
\hline 20 & CONSOLIDATE & 1 & 401 & $0.2 \%$ \\
\hline 21 & ENDURE & 1 & 517 & $0.2 \%$ \\
\hline 22 & STIMULATE & 1 & 1027 & $0.1 \%$ \\
\hline 23 & STRENGTHEN & 1 & 1193 & $0.1 \%$ \\
\hline 24 & ENGAGE & 1 & 1166 & $0.1 \%$ \\
\hline 25 & SUSTAIN & 1 & 1225 & $0.1 \%$ \\
\hline 26 & ABANDON & 1 & 1294 & $0.1 \%$ \\
\hline 27 & TRIUMPH & 1 & 1777 & $0.1 \%$ \\
\hline 28 & DEFEND & 1 & 2021 & $0.0 \%$ \\
\hline 29 & SUCCEED & 1 & 2102 & $0.0 \%$ \\
\hline 30 & YIELD & 1 & 2101 & $0.0 \%$ \\
\hline 31 & EXTEND & 1 & 3101 & $0.0 \%$ \\
\hline 32 & INDICATE & 1 & 4097 & $0.0 \%$ \\
\hline 33 & DEPEND & 1 & 3450 & $0.0 \%$ \\
\hline 34 & DETERMINE & 1 & 3900 & $0.0 \%$ \\
\hline 35 & REPLACE & 1 & 3373 & $0.0 \%$ \\
\hline 36 & PUSH & 1 & 3506 & $0.0 \%$ \\
\hline 37 & PREFER & 1 & 3644 & $0.0 \%$ \\
\hline 38 & RULE & 1 & 9241 & $0.0 \%$ \\
\hline 39 & REQUIRE & 1 & 6836 & $0.0 \%$ \\
\hline 40 & REMAIN & 1 & 8894 & $0.0 \%$ \\
\hline 41 & CHOOSE & 1 & 6712 & $0.0 \%$ \\
\hline 42 & PREVENT & 1 & 6676 & $0.0 \%$ \\
\hline 43 & JOIN & 1 & 7275 & $0.0 \%$ \\
\hline 44 & GO & 1 & 87209 & $0.0 \%$ \\
\hline 45 & SHOW & 1 & 26209 & $0.0 \%$ \\
\hline 46 & NEED & 1 & 54587 & $0.0 \%$ \\
\hline 47 & GET & 1 & 96147 & $0.0 \%$ \\
\hline 48 & WORK & 1 & 89441 & $0.0 \%$ \\
\hline 49 & FORCE & 1 & 15460 & $0.0 \%$ \\
\hline \multirow[t]{2}{*}{50} & SEE & 1 & 115200 & $0.0 \%$ \\
\hline & TOTAL & 87 & & \\
\hline
\end{tabular}


Table n. 3: "Relationships signs" in American Sign Language. (Learning Sign Language)

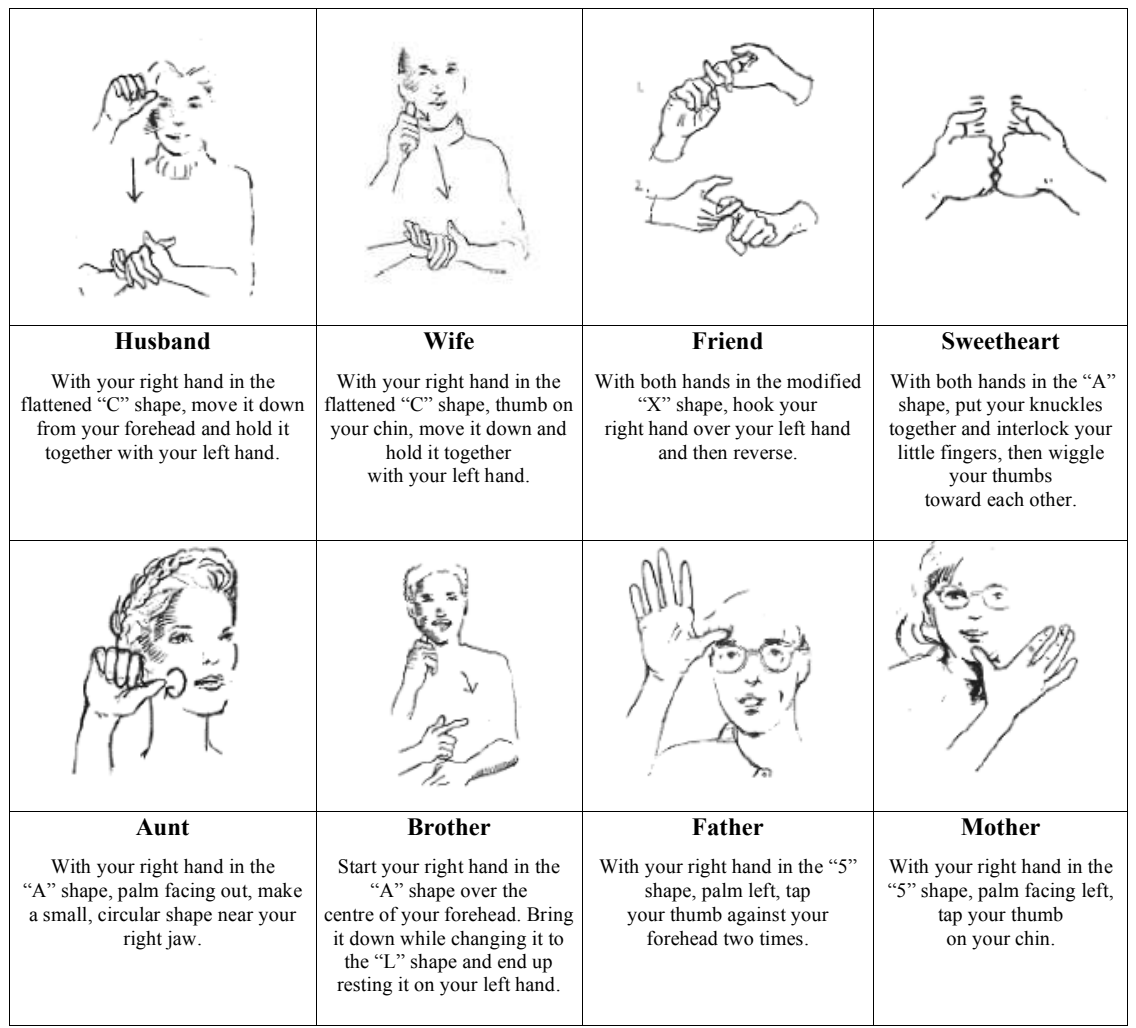

\section{Works Cited}

"Conceptual Metaphor" anwers.com <www.anwers.com/topic/ conceptual_metaphor $>(12 / 08)$.

Boers, Frank. "Metaphor Awarness and Vocabulary Retention". Applied Linguistics 21/4 (2000): 553 -571.

Brandt, Per Aage. "Mental Spaces and Cognitive Semantics: A Critical Comment." Journal of Pragmatics 37 (2005): 1578-1594.

Cambrige International Dictionary of English. Cambridge: University Press, 1995.

Cooper, David E. Metaphor. Oxford: Basic Blackwell, 1986

Davies, Mark. (2004-) BYU-BNC: The British National Corpus. Available online at $<$ http://corpus.byu.edu/bnc $>(03 / 09)$

Deignan, Alice, D. Gabryś and A. Solska. "Teaching English Metaphors Using Cross-linguistic Awareness-raising Activity." ELT Journal 51/4 (1997): 352-360.

Deignan, Alice. "A Corpus Linguistic Perspective on the Relationship Betwe- 
en Metonymy and Metaphor". Style. 39/1 (2004): .72-91.

Díez Velasco, Olga Isabel. "The Role of Semantic Relations in The Creation of Metonymic Mappings" in Miscelánea: A Journal of English and American studies, 23 (2001): 11-28.

Fass, Dan. "Metonymy and Metaphor: What's the Difference?”. Proceedings of the $12^{\text {th }}$ Conference on Computational Linguistics 1988: 177-181.

Gibbs, Raymond W. and Gerard J. Steen: Metaphor in cognitive linguistics : selected papers from the fifth international cognitive linguistics conference. Amsterdam: Benjamins, 1999.

Greenbaum, Sidney and Randolph Quirk. A Student's Grammar of the English Language. London: Longman, 1990.

IL, Vocabolario della Lingua Latina (Castiglione-Mariotti). Torino: Loescher, 1986. Jackendoff, Ray. Linguaggio e Natura Umana. (Patterns in the mind. Language and Human Nature). Trans. Alberto Peruzzi. Bologna: Il Mulino, 1998.

Lakoff, George and Mark Johnson. Metaphors we live by. Chicago: Chicago University Press, 1980.

Lakoff, George. Women, Fire, and Dangerous Things: What Categories Reveal about the Mind. Chicago. University of Chicago Press, 1987.

Lakoff, George. "The Contemporary Theory of Metaphor" in A. Ortony (ed.) Metaphor and Thought. Cambridge: Cambridge University Press, 1992, pp. 202-251.

Language Learning: ASL for beginners. Laser Publishing Group, CD-ROM.

Shelly, Susan and Jim Schneck. The Complete Idiot's Guide to Learning Sign Language. New York: Alpha Books, 1998.

Steen, Gereard. "Metaphor: Stylistic Approaches". In Keith Brown (ed.), The Encyclopedia of Language and Linguistics, $2^{\text {nd }}$ ed., Vol. 8, 51-58. Oxford: Elsevier. 2006.

Stefanowitsch, Anatol and Stefan Th. Gries. Corpus-based approaches to metaphor and metonymy. Berlin: Mouton de Gruyter, 2006.

View: Variation In English Words and Phrases. See Davies, Mark, above.

Webster's College Dictionary. New York: Random House, 1990.

Note

${ }^{1}$ Il presente contributo rappresenta la relazione consegnata dall'autrice alla fine di un corso di lingua inglese per la laurea specialistica all'Università di Firenze. Il corso viene descritto in questo volume nel contributo "Ordinary language metaphors: a plan for a corpus-based research project", di Scott Staton (nota del curatore).

${ }^{2}$ From the definition of "Conceptual metaphor". Answers.com.

${ }^{3}$ Ibid.

${ }^{4}$ A database interface elaborated by Mark Davis at the Brigham Young University $<$ http://view.byu.edu/>.

${ }^{5}$ Complete Lexical Tour, < http://132.203.2224.131>, or Michigan Corpus of Academic Spoken English, <http://micase.umdl.umich.edu/>.

${ }^{6}$ The database is very good, but it does not list more than one thousand entries for each query. 
7 Table n.1 in the Appendix shows what a "comparative window" looks like.

${ }^{8}$ As example I have used the plural form alliances in order to have less entries; however, the singular form alliance gives the same verbs as main entries.

${ }^{9}$ One of the Lakoff basic-source categories. See Lakoff, Women, Fire, and Dangerous Things p. 406.

${ }^{10}$ In the dictionary the verb form has thirteen entries: eight of them have the literal meaning "to make or produce"(Webster's), while the other five have shifted into a metaphorical meaning. Already in Latin the verb underwent this metaphorical shift: the main meaning of "FORMARE" is "to shape", "to sculpt" : materiam fingere et formare (Cic.), while the last entries concern a metaphorical use: puerum dictis formare or formare versus cithara $\bar{a}$ (IL). As to the verb make in the transitive form, it has thirty-two entries and the main entries have the literal meaning "to bring into existence by shaping, changing, or combining material"'(Webster's).

11 "An object whose referent exists only by virtue of the activity indicated by the verb" ( Greenbaum and Quirk, A Student's Grammar of the English Language, p. 212).

${ }^{12}$ Definitions of "forge" and "build". Webster's College Dictionary.

${ }^{13}$ And not BUILDING as some linguistics affirm. Indeed, for example, it is possible to build an unlimited number of identical buildings, whereas relationships, as artefacts, are different from each other.

${ }^{14}$ I use the same scheme applied by Lakoff in Women, Fire, and Dangerous Things.

${ }^{15}$ In the section "Some Clarifications" I will better explain that this is the correct way in which the participant is conceptualised.

${ }^{16}$ As relationship components I mean feelings such as faith, respect, love, or the same people that are involved in the relationship. I will better explain the semantic role of the subjects in the section "Some Clarifications".

${ }^{17}$ View does not provide more entries.

${ }^{18}$ A "linguistic metaphor" is "the spoken or written realization of a conceptual metaphor" (Deignan, Gabryś, and Solska, p. 352)

${ }^{19}$ I have not used the word "metaphor", because most of the time the addressee understands metaphors without identifying them as metaphors.

${ }^{20} \mathrm{It}$ is proved that ASL is an independent language, it is not generated by "verbal languages" (Jackendoff).

${ }^{21}$ I do not use "agentive and affected subject" in a strict grammatical sense: they are used in order to better explain my thesis.

${ }^{22}$ This is an example given by Lakoff to explain one of the basic kinesthetic image schemas: the CONTAINER schema. If we analyse the conceptual metaphor RELATIONSHIP ARE ARTEFACTS in the light of Lakoff's kinesthetic image schemas, we realise that it satisfies most of them: the PART-WHOLE schema, the CENTER-PERIPHERY schema, the source-PATH-GoAl schema, etc. (Women, Fire, and Dangerous Things) 


\title{
BREVE STORIA LEGISLATIVA, GIURIDICA \\ E CONTRATTUALE DEGLI INSEGNANTI UNIVERSITARI \\ DI MADRELINGUA IN ITALIA
}

\author{
John Gilbert \\ Università di Firenze
}

\section{Premessa}

Prima di tutto vorrei ringraziare i colleghi che hanno organizzato questo convegno per avermi invitato a parlare oggi. L'obiettivo della mia relazione è di fornire una contestualizzazione storica per la discussione che si svolgerà oggi sull'evoluzione del ruolo dell'insegnante universitario di lingua, e più precisamente della figura del lettore di madrelingua. Infatti, qualsiasi considerazione sull'insegnamento delle lingue nelle università non può prescindere dal ruolo fondamentale svolto da questi insegnanti di madrelingua, responsabili storicamente, ed ancora oggi, della quasi totalità dell'insegnamento linguistico negli atenei italiani. Nel tempo limitato a mia disposizione cercherò di tracciare in maniera purtroppo schematica i momenti salienti nella storia travagliata di questa figura, dalle sue origini fino ai giorni nostri, una storia assai complessa ed articolata e quindi mi scuso in anticipo per eventuali semplificazioni ed omissioni. Infine cercherò di indicare delle soluzioni concrete ed immediate per sanare la situazione attuale e potenziare la formazione linguistica negli atenei italiani.

\section{La storia}

Nel 1958 la figura del lettore di madrelingua viene equiparata a quella dell'assistente universitario con la Legge 349. L'identità tra lettori ed assistenti continua poi fino al 1980 quando è approvata la Legge 382 per il Riordinamento della docenza universitaria. La nuova Legge 382 mette ad esaurimento la figura dell'assistente universitario e istituisce la nuova figura del ricercatore universitario. Nello stesso tempo l'art. 28 della Legge 382 crea una nuova figura di lettore di madrelingua che non viene più equiparata alla precedente figura dell'assistente e non gode più di un rapporto di lavoro subordinato di diritto pubblico a tempo indeterminato come prima. La nuova figura ha invece un rapporto di lavoro di 
diritto privato come libero professionista senza trattamento assistenziale e previdenziale con un trattamento economico deciso da ogni singolo ateneo e comunque non superiore allo stipendio iniziale del professore associato a tempo definito (ovvero tempo parziale) e con contratti individuali annuali che non possono essere rinnovati per più di cinque anni con lo stesso ateneo.

In questa maniera si inizia la lunga storia del trattamento discriminatorio della categoria maggiormente responsabile per l'insegnamento delle lingue nelle università italiane, con un rapporto di lavoro precario ai danni della continuità didattica e agli interessi degli studenti. Purtroppo questo atteggiamento è assai indicativo della scarsa considerazione data all'insegnamento linguistico in Italia per non dire ai diritti di una categoria di insegnanti in gran parte cittadini stranieri.

Nella prima parte degli anni '80, i lettori insegnavano quasi sempre all'interno di corsi di lingua e letteratura straniera dove il titolare del corso era un professore di letteratura mentre la glottodidattica rappresentava una specie di cugino povero della letteratura, con le lingue insegnate dai lettori. Nel resto dell'Europa, spesso la figura dell'insegnante di madrelingua esiste ma viene utilizzata in maniera diversa. In paesi come la Germania, la Francia e la Spagna un numero assai ristretto di figure di madrelingua generalmente affianca un numero ben superiore di professori mentre in Italia la situazione è l'opposto. A partire dagli anni '80 e fino ad oggi ci sono sempre stati relativamente pochi professori o ricercatori di lingua e numeri ben più alti di insegnanti di madrelingua, circa 2.000 negli atenei italiani oggi.

Negli anni '80 e '90 vengono creati i primi centri linguistici nelle università italiane e spesso i lettori delle facoltà, soprattutto le facoltà scientifiche, vengono trasferiti nei nuovi centri. I relatori che seguiranno in mattinata parleranno in maniera specifica dell'evoluzione dell'insegnamento linguistico in questi centri negli ultimi venti anni.

A causa delle condizioni discriminatorie di lavoro, i primi lettori cominciavano ad organizzarsi e sindacalizzarsi già verso la metà degli anni '80, iniziando ricorsi legali presso le corti italiane e la Corte di giustizia europea, ricorsi che negli anni avrebbero coinvolto più di mille insegnanti. Trattandosi di una prestazione di lavoro da libero professionista, spesso i lettori venivano pagati in ritardo e solo poche volte all'anno. A Firenze, per esempio, nei primi anni i lettori venivano pagati solo 2 volte all'anno. Non esisteva il diritto alla maternità e le lettrici spesso dovevano insegnare fino all'ottavo o nono mese per poi dover riprendere i corsi in tempi brevi dopo il parto. Cominciavano le prime agitazioni e i blocchi della didattica e degli esami, spesso con il sostegno degli studenti; basta come esempio ricordare la solidarietà dimostrata in molti atenei dal movimento studentesco de "la Pantera" nel'89 e '90. 
Verso la fine degli anni '80 molti lettori cominciavano a raggiungere il limite del numero di rinnovi contrattuali possibili nella loro università e dovevano cercare altri lavori o altri atenei. Nel 1989 una sentenza della Corte costituzionale ha tolto il limite al numero di rinnovi contrattuali presso lo stesso ateneo ma era ormai troppo tardi per tanti di quelli che non avevano avuto rinnovato il loro contratto.

Nel 1989 è arrivata la prima sentenza della Corte di giustizia europea che ha condannato il trattamento discriminatorio dei lettori. La sentenza ha riconosciuto la natura di lavoro subordinato e non di libero professionista e il diritto a contratti a tempo indeterminato. Così le università erano costrette ad assumere i lettori come dipendenti, portatori di diritti come gli altri lavoratori, incluso il diritto al trattamento assistenziale e previdenziale, ma continuavano comunque ad assumere i lettori con contratti precari annuali nonostante la sentenza. Poi nel 1993 è arrivata una seconda sentenza di condanna dalla Corte di giustizia europea per la mancata applicazione della prima sentenza in termini di contratti a tempo indeterminato. A questo punto l'Italia fu costretta a rispettare la sentenza ma la maggior parte degli atenei non hanno trasformato i contratti dei lettori in servizio in contratti a tempo indeterminato (a Firenze, però, sì). Invece hanno lasciato scadere i contratti annuali alla fine dell'anno accademico '92-'93 e hanno costretto i lettori precedentemente in servizio a partecipare ad una nuova selezione pubblica secondo le norme di una serie di nuovi decreti di legge che hanno abrogato l'art. 28 della Legge 382 del 1980 (che aveva previsto il ruolo del lettore) e hanno creato una nuova figura chiamata "collaboratore ed esperto linguistico" ovvero c.e.l. I lettori che rifiutavano di rifare una selezione pubblica per una posizione come c.e.l. venivano licenziati in diversi atenei (a Bologna, Federico II di Napoli, Napoli Orientale, Salerno, Verona). In seguito la maggior parte di questi insegnanti sono stati reintegrati ai posti di lavoro con sentenze della magistratura.

Questi decreti a partire dal 1993 sarebbero poi diventati l'art. 4 della Legge 236 nel 1995, una legge che rappresenta un palese tentativo di eludere la giurisprudenza italiana ed europea sopprimendo la figura del lettore di madrelingua e creando ope legis la nuova figura del c.e.l., senza il riconoscimento dei diritti acquisiti degli ex-lettori. In risposta ci sono state 3 risoluzioni di condanna del Parlamento europeo, nel 1995, 1996 e 2000. Infine la Legge 236 prevede anche una verifica annuale del lavoro svolto dal c.e.l. e la continuità del rapporto di lavoro del c.e.l. è subordinata al giudizio positivo di quella verifica. Una verifica annuale che può portare al licenziamento non è prevista per nessun'altra categoria di lavoratori all'università o anche nel pubblico impiego in generale.

Nel 1996 veniva firmato il primo contratto collettivo nazionale di lavoro per il comparto università con un articolo specifico per gli exlettori e i nuovi c.e.l., l'art. 51. Più di mille lettori avevano firmato un 
documento chiedendo l'inserimento nel nuovo contratto nazionale del comparto università, ma i contenuti normativi e il trattamento economico rappresentavano un enorme passo indietro rispetto alle condizioni di lavoro già esistenti (e già insoddisfacenti) nella maggior parte degli atenei. Inoltre il nuovo contratto nazionale stabiliva solo un trattamento economico minimo, rimandando la questione alla contrattazione decentrata di ateneo. Già nel 1993 il primo contratto collettivo di ateneo era stato firmato a Firenze e negli anni, con le firme di un numero crescente di contratti di ateneo, le condizioni di lavoro e il trattamento economico cominciavano a differenziarsi sempre di più. Nel 1997 ci fu poi una terza sentenza di condanna da parte della Corte di Giustizia europea per aver escluso dei lettori dalla possibilità di fare domande per supplenze.

Nel 1999 la legge 509 per la Riforma della Didattica ha stabilito il nuovo sistema " 3 più 2", con la nuova laurea triennale e quella specialistica biennale. Almeno per le lingue principali la letteratura viene finalmente separata dalla lingua, come nel resto del mondo. Per la prima volta con i nuovi ordinamenti la conoscenza (e la relativa verifica) orale e scritta di almeno una lingua europea è richiesta da tutti i nuovi corsi di laurea. Alcuni corsi di laurea triennale richiedono la padronanza di due lingue straniere e la conoscenza anche del patrimonio culturale delle civiltà di cui queste lingue sono espressione, oltre ad un'eventuale sufficiente competenza scritta e orale in una terza lingua. Anche nelle lauree specialistiche sono previsti simili requisiti. La legge poi auspica "l'europeizzazione" dei curricula e l'internazionalizzazione delle formazioni offerte. Però nella nuova riforma della didattica non appare mai il termine lettore/cel di madrelingua nonostante il fatto che quella figura sia indispensabile per raggiungere gli obiettivi didattici previsti dalla riforma nel settore della formazione linguistica.

Sempre nel 1999 più di mille docenti universitari sottoscrivono un Appello del mondo accademico per "Riconoscere i lettori come personale insegnante in vista del nuovo contratto nazionale 1998-2001", un appello da parte della docenza universitaria per riconoscere la professionalità e la dignità del lettore di madrelingua come personale insegnante.

Successivamente nel 2001 ci fu una quinta sentenza di condanna dalla Corte di Giustizia europea per il mancato riconoscimento dei diritti acquisiti dei lettori. Poi nel corso delle trattative per il rinnovo del contratto nazionale del comparto università 1998-2001 ci fu un parziale accordo nel febbraio 2000 per ciò che riguardava la definizione del mansionario dei c.e.l. ed il trattamento economico, poi smentito e ritirato dalla controparte. Ai lettori/c.e.l., diversamente da tutto il resto del pubblico impiego, non è stato riconosciuto nessun aumento salariale per il biennio economico 1998-1999. In seguito il biennio economico del contratto nazionale 2000-2001 (siglato 13.05.03) ha inserito un articolo specifico sui c.e.l., l'art. 22 , che incredibilmente ha rinviato alla contrattazione decen- 
trata di ateneo l'applicazione delle sentenze della Corte di Giustizia europea in materia di diritti acquisiti dei lettori, con un aumento economico totalmente insoddisfacente. Nello stesso tempo quel contratto nazionale ha creato una nuova figura, il tecnico-linguistico per svolgere "attività strumentali di supporto all'apprendimento linguistico", da inserire fra le categorie del personale tecnico-amministrativo con posti riservati ai c.e.l. nei nuovi concorsi. La strategia di una parte della Conferenza dei Rettori (CRUI) è ormai palese: dopo il tentativo di sopprimere i lettori e sostituirli con i c.e.l., adesso si tenta di eliminare i c.e.l. trasformandoli in personale tecnico-amministrativo con la nuova figura del t.l. ovvero il tecnico-linguistico, come si sta già facendo in particolar modo a $\mathrm{Pa}-$ dova e a Viterbo (ateneo del segretario della CRUI).

In data 4 marzo 2004 la Commissione europea ha riferito l'Italia di nuovo alla Corte di Giustizia europea per la mancata esecuzione da parte dello Stato italiano della sentenza della stessa Corte del 2001 riguardante i diritti acquisiti degli ex lettori con la richiesta del pagamento di una multa di oltre 300.000 euro al giorno finché non venisse applicata la sentenza. La risposta del Governo italiano è stata la Legge 63 del 2004 che prevedeva, proporzionalmente all'impegno orario assolto, tenendo conto che l'impegno pieno corrisponde a 500 ore, un trattamento economico corrispondente a quello del ricercatore confermato a tempo definito (ovvero tempo parziale) esclusivamente per gli ex lettori di solo 6 atenei (la Basilicata, Milano, Palermo, Pisa, La Sapienza di Roma e L'Orientale di Napoli). In seguito alcune sentenze della Corte di Cassazione (n.21856/2004 e 5909/2005) hanno esteso l'ambito di applicazione della legge a tutti gli atenei italiani ed anche ai c.e.l. non ex lettori.

Poi il 18 luglio 2006 la Corte di Giustizia europea ha rilasciato una nuova sentenza di condanna dell'Italia, con il pagamento delle spese legali, per non aver applicata la sentenza del 2001 entro i tempi indicati dalla Commissione. Però nello stesso tempo la Corte ha dichiarato che, contrariamente al parere della Commissione, con l'applicazione della Legge 63/2004 l'inadempimento dell'Italia non persisteva e quindi ha respinto la richiesta dell'applicazione della multa. Il punto fondamentale emerso dalla sentenza comunque è che la Corte ha finalmente stabilito una base minima garantita a livello nazionale per il parametro di retribuzione e per il monte ore: per un rapporto di lavoro a tempo pieno, ovvero 500 ore, viene riconosciuto almeno il parametro economico del ricercatore a tempo definito. Tuttavia questo parametro economico minimo continua a non essere riconosciuto né dal contratto nazionale di lavoro né dalla maggior parte dei contratti integrativi di ateneo e in ogni caso non rappresenta il parametro economico indicato da gran parte della giurisprudenza in Italia, ovvero quello del ricercatore confermato a tempo pieno.

Inoltre esiste ancora un problema gravissimo di precarietà fra i lettori/ c.e.l. Oggi circa un quarto della categoria, ovvero circa 500 insegnanti 
(fonte MIUR, 2003), è ancora precario con contratti a tempo determinato in palese violazione dell'art. 4 della Legge 236/95 che prevede di norma il rapporto di lavoro a tempo indeterminato, con contratti a tempo determinato solo per esigenze temporanee. Inutile evidenziare i seri danni recati alla formazione linguistica degli studenti universitari a causa del persistere di questa situazione di incertezza e sofferenza da più di venticinque anni. Bisogna stabilizzare questi insegnanti e il Decreto del precedente Ministro Nicolais che riguarda l'attuazione delle Leggi Finanziarie per il 2007 e il 2008 per la stabilizzazione dei lavoratori precari nelle università ci fornisce lo strumento necessario: adesso che si può farla, bisogna verificare la reale volontà degli atenei di migliorare l'offerta formativa linguistica stabilizzando i troppi lettori/cel precari.

Oggi, mentre si aggrava l'emergenza per l'apprendimento delle lingue straniere in Italia, i lettori/c.e.l. di madrelingua sono ormai da circa 30 mesi senza il rinnovo del contratto nazionale di lavoro. La posizione ufficiale degli attuali rappresentanti della Conferenza dei rettori (CRUI) sta bloccando le trattative purtroppo, perchè pretendono che il contratto nazionale di lavoro trasformi la figura del lettore/c.e.l. in quella del tecnico-linguistico inquadrato fra le categorie del personale tecnico-amministrativo.

Bisogna senz'altro dare una definizione definitiva dello stato giuridico dei lettori/cel dopo più di 25 anni, ma quella definizione non potrà certamente prescindere dal pieno riconoscimento professionale della funzione didattica svolta da questa figura, il cui compito primario è l'insegnamento della propria lingua madre come lingua straniera. Come già riconosciuto dagli organi europei, non si tratta della figura di un lavoratore tecnico-amministrativo ma bensì di un insegnante. L'accanito rifiuto di riconoscere questa semplice verità continuerà a portare seri danni alla formazione linguistica a livello universitario - e non solo lì, se consideriamo, per esempio, il ruolo che quella formazione gioca nella preparazione degli insegnanti di lingua nelle nostre scuole di domani.

Purtroppo in troppi atenei è in corso ormai da qualche anno un tentativo di de-mansionamento delle attività didattiche svolte dai lettori/c. el. mentre troppo spesso si ricorre all'esternalizzazione, dando in appalto all'esterno la formazione linguistica e anche le verifiche, alle scuole private di lingua, alle agenzie formative, alle fondazioni, alle convenzioni, dove non si può garantire il controllo e la qualità che la formazione pubblica dovrebbe assicurare.

In alcuni atenei invece si tenta di risolvere il problema della titolarità degli insegnamenti con il ricorso ai professori a contratto. Questi contratti con figure estranee agli atenei e sottopagate non possono offrire il numero di ore di didattica che viene offerto da un contratto di lettorato e spesso vengono stipulati con persone non di madrelingua e comunque di norma con persone (p.e. i nostri studenti neo-laureati o chi non è ri- 
uscito ad entrare in graduatoria come insegnante) che non possiedono il patrimonio di esperienza didattica e scientifica dei lettori.

Ma la questione della titolarità dei corsi di lingua non dovrebbe rappresentare più un problema dopo l'invio di due note nell'estate del 2006 (Nota del 28.06 .06 prot. 1232 e nota del 2.08 .06 prot. 1430) da parte del Ministero dell'Università e della Ricerca a tutti gli atenei italiani per informarli che i lettori hanno diritto a partecipare ai bandi per gli affidamenti e le supplenze. Questa novità rappresenta una importantissima occasione per il miglioramento della offerta di formazione linguistica negli atenei italiani, novità che potrebbe risolvere in gran parte la mancanza di professori e ricercatori di lingua nelle facoltà e che potrebbe superare la pratica fallimentare dell'utilizzo di contratti di insegnamento a personale esterno per le esigenze di formazione e verifica linguistica nei corsi di laurea. Già dall'anno accademico 2006-7 diversi atenei in Piemonte, Liguria, Puglia, Lazio ed altrove hanno iniziato a coprire i loro corsi di lingua - sempre previa autorizzazione dei relativi organi decisionali - con affidamenti ai propri lettori/cel. Tuttavia troppi atenei, la maggioranza, continuano ad escludere a priori i lettori/cel dai conferimenti di affidamenti e supplenze in palese contrasto con le indicazioni del precedente Ministero. Invece ci sono tutte le premesse necessarie per dimostrare una vera volontà di affrontare la grave crisi della formazione linguistica che allontana l'Italia da quel plurilinguismo che caratterizza il resto dell'Unione europea. Il problema dei lettori/cel di madrelingua è solo un aspetto di quella crisi ma certamente è emblematico e come anello nel ciclo formativo è un aspetto sicuramente centrale.

\section{Conclusioni}

Per concludere, da tanti anni ormai esiste una "emergenza lingue straniere" in Italia; l'ultima denuncia della situazione da parte della Conferenza dei Presidi delle Facoltà di Lingue e Letterature Straniere risale appena al 15 marzo scorso. In Italia persiste un monolinguismo di fatto che pregiudica il ruolo culturale, politico ed economico del paese in Europa e nel mondo. Questo analfabetismo linguistico è in netto contrasto con le esigenze della moderna società della conoscenza. Per capire le molteplici ragioni che hanno portato l'Italia ad essere il fanalino di coda dell'Europa per ciò che riguarda le competenze linguistiche dei suoi cittadini sarebbe necessario analizzare le carenze dell'insegnamento delle lingue straniere attraverso tutto il percorso formativo, dalla scuola dell'infanzia all'alta formazione universitaria.

E se consideriamo la formazione linguistica nelle università, allora qualsiasi considerazione non può prescindere dal ruolo fondamentale svolto dagli insegnanti di madrelingua. Come avevo già notato nella 
mia introduzione, i lettori/c.e.l. sono ancora oggi la figura docente responsabile per la quasi totalità dell'insegnamento linguistico negli atenei italiani e sarebbe difficile negare che dal 1980 ad oggi la mancata definizione dello stato giuridico e il non riconoscimento del ruolo professionale effettivamente svolto da questi insegnanti hanno gravemente compromesso la qualità dell'offerta formativa degli atenei in termini di formazione linguistica, con conseguenti ripercussioni su tutto il resto del ciclo formativo.

I lettori/c.e.l. dedicano circa un milione di ore di didattica ogni anno alla formazione linguistica negli atenei, svolgendo autonomamente i loro corsi nelle facoltà e nei centri linguistici in corsi di lingua ad ogni livello, sia di lingua generale che di lingua specialistica, inclusa la relativa programmazione, didattica e valutazione, e partecipando alle commissioni d'esame per gli esami di profitto nelle facoltà di lingue, lettere e filosofia, economia, scienze politiche e scienza della formazione e agli esami di idoneità linguistica nelle facoltà scientifiche.

Tuttavia sono ancora senza un profilo professionale definito, né per legge né per contratto nazionale di lavoro, una figura ancora oggi - dopo più di 25 anni - sottopagata e mortificata con un trattamento normativo ed economico del tutto disomogeneo a livello nazionale. Lo stipendio suggerito dal contratto nazionale mette loro ben al di sotto della "soglia di povertà " indicata dai dati ISTAT. Ed è normale che quando un insegnante non ha la possibilità di percepire uno stipendio decoroso e si trova in uno stato di precarietà perenne, quando vede mortificata la sua dignità professionale ed umana da oltre 20 anni, non ha quella tranquillità di esistenza assolutamente necessaria per poter dare il meglio di sé nelle aule delle università italiane. Avviene una sorta di instabilità occupazionale istituzionalizzata. Ovviamente questo stato di cose non può che avere una ripercussione negativa sulla formazione linguistica fornita agli studenti universitari. Bisogna finalmente risolvere questo problema strutturale della formazione linguistica in Italia, negli interessi soprattutto degli studenti come titolari di diritti, in primo luogo del diritto a servizi adeguati forniti dall'Università pubblica. Un'altra università è possibile se la vogliamo davvero. 

